\title{
Exploring cosmic origins with CORE : Mitigation of systematic effects
}

\section{CORE Collaboration}

2018-04

CORE Collaboration, Natoli , P , Kiiveri , K , Lindholm , V , Keihänen , E , Kurki-Suonio , H \& Väliviita , J 2018 , ' Exploring cosmic origins with CORE : Mitigation of systematic effects ' , Journal of Cosmology and Astroparticle Physics , no. 4 , 022 . https://doi.org/10.1088/1475-7516/2018/04/022

http://hdl.handle.net/10138/308567

https://doi.org/10.1088/1475-7516/2018/04/022

unspecified

acceptedVersion

Downloaded from Helda, University of Helsinki institutional repository.

This is an electronic reprint of the original article.

This reprint may differ from the original in pagination and typographic detail.

Please cite the original version. 


\section{Exploring cosmic origins with CORE: mitigation of systematic effects}

P. Natoli, ${ }^{1,2, a}$ M. Ashdown, ${ }^{3,4}$ R. Banerji, ${ }^{5}$ J. Borrill, ${ }^{6,7}$ A. Buzzelli, ${ }^{8,9,10}$ G. de Gasperis, ${ }^{9,10}$ J. Delabrouille, ${ }^{5}$ E. Hivon, ${ }^{11}$ D. Molinari, ${ }^{1,2,12}$ G. Patanchon, ${ }^{5}$ L. Polastri, ${ }^{1,2}$ M. Tomasi, ${ }^{13,14}$ F. R. Bouchet, ${ }^{11}$

S. Henrot-Versillé, ${ }^{15}$ D. T. Hoang, ${ }^{5}$ R. Keskitalo, ${ }^{6,7}$ K. Kiiveri, ${ }^{16,17}$

T. Kisner, ${ }^{6}$ V. Lindholm, ${ }^{16,17}$ D. McCarthy, ${ }^{18}$ F. Piacentini, ${ }^{8,19}$

O. Perdereau, ${ }^{15}$ G. Polenta, ${ }^{20,21}$ M. Tristram, ${ }^{15}$ A. Achucarro, ${ }^{22,23}$

P. Ade, ${ }^{24}$ R. Allison, ${ }^{25}$ C. Baccigalupi, ${ }^{26,27}$ M. Ballardini, ${ }^{12,28,29}$

A. J. Banday, ${ }^{30,31}$ J. Bartlett, ${ }^{5}$ N. Bartolo, ${ }^{32,33,34}$ S. Basak, ${ }^{35,26}$

J. Baselmans, ${ }^{36,37}$ D. Baumann, ${ }^{38}$ M. Bersanelli, ${ }^{13,14}$ A. Bonaldi, ${ }^{39}$

M. Bonato, ${ }^{40,26}$ F. Boulanger, ${ }^{41}$ T. Brinckmann, ${ }^{42}$ M. Bucher, ${ }^{5}$

C. Burigana, ${ }^{12,1,29}$ Z.-Y. Cai, ${ }^{43}$ M. Calvo, ${ }^{44}$ C.-S. Carvalho, ${ }^{45}$

G. Castellano, ${ }^{46}$ A. Challinor, ${ }^{38}$ J. Chluba, ${ }^{39}$ S. Clesse, ${ }^{42}$

I. Colantoni, ${ }^{46}$ A. Coppolecchia, ${ }^{8,19}$ M. Crook, ${ }^{47}$ G. D'Alessandro, ${ }^{8,19}$

P. de Bernardis, ${ }^{8,19}$ G. De Zotti, ${ }^{34}$ E. Di Valentino, ${ }^{11,48}$ J.-M. Diego, ${ }^{49}$

J. Errard, ${ }^{50}$ S. Feeney, ${ }^{3,51}$ R. Fernandez-Cobos, ${ }^{49}$ F. Finelli, ${ }^{12,29}$

F. Forastieri, ${ }^{1,2}$ S. Galli, ${ }^{11}$ R. Genova-Santos, ${ }^{52,53}$ M. Gerbino, ${ }^{54,55}$

J. González-Nuevo, ${ }^{56}$ S. Grandis, ${ }^{57,58}$ J. Greenslade, ${ }^{3}$

A. Gruppuso, ${ }^{12,29,1}$ S. Hagstotz, ${ }^{57,58}$ S. Hanany, ${ }^{59}$ W. Handley, ${ }^{3,4}$

C. Hernandez-Monteagudo, ${ }^{60}$ C. Hervías-Caimapo, ${ }^{39}$ M. Hills, ${ }^{47}$

E. Keihänen, ${ }^{16,17}$ T. Kitching, ${ }^{61}$ M. Kunz, ${ }^{62}$ H. Kurki-Suonio, ${ }^{16,17}$

L. Lamagna, ${ }^{8,19}$ A. Lasenby, ${ }^{3,4}$ M. Lattanzi, ${ }^{2,1}$ J. Lesgourgues, ${ }^{42}$

A. Lewis, ${ }^{63}$ M. Liguori, ${ }^{32,33,34}$ M. López-Caniego, ${ }^{64}$ G. Luzzi, ${ }^{8}$

B. Maffei, ${ }^{41}$ N. Mandolesi, ${ }^{1,12}$ E. Martinez-González, ${ }^{49}$

C.J.A.P. Martins, ${ }^{65}$ S. Masi, ${ }^{8,19}$ A. Melchiorri, ${ }^{8,19}$ J.-B. Melin, ${ }^{66}$

M. Migliaccio ${ }^{20,9}$ A. Monfardini, ${ }^{44}$ M. Negrello, ${ }^{24}$ A. Notari, ${ }^{67}$

L. Pagano, ${ }^{41}$ A. Paiella, $,{ }^{9}, 19$ D. Paoletti, ${ }^{12}$ M. Piat, ${ }^{5}$ G. Pisano, ${ }^{24}$

A. Pollo, ${ }^{68}$ V. Poulin, ${ }^{42,69}$ M. Quartin, ${ }^{70,71}$ M. Remazeilles, ${ }^{39}$

M. Roman, ${ }^{72}$ G. Rossi, ${ }^{73}$ J.-A. Rubino-Martin, ${ }^{52,53}$ L. Salvati, ${ }^{9,19}$

G. Signorelli, ${ }^{74}$ A. Tartari, ${ }^{5}$ D. Tramonte, ${ }^{52}$ N. Trappe, ${ }^{18}$

T. Trombetti, ${ }^{12,1,29}$ C. Tucker, ${ }^{24}$ J. Valiviita, ${ }^{16,17}$ R. Van de Weijgaert, ${ }^{75,76}$

B. van Tent, ${ }^{77}$ V. Vennin, ${ }^{78}$ P. Vielva, ${ }^{49}$ N. Vittorio,,${ }^{9,10}$ C. Wallis, ${ }^{39}$

K. Young, ${ }^{59}$ and M. Zannoni ${ }^{79,80}$ for the CORE collaboration. 
${ }^{1}$ Dipartimento di Fisica e Scienze della Terra, Università di Ferrara, Via Saragat 1, 44122 Ferrara, Italy

${ }^{2}$ INFN, Sezione di Ferrara, Via Saragat 1, 44122 Ferrara, Italy

${ }^{3}$ Astrophysics Group, Cavendish Laboratory, University of Cambridge, J. J. Thomson Avenue, Cambridge, CB3 OHE, UK

${ }^{4}$ Kavli Institute for Cosmology, Univerisity of Cambridge, Madingley Road, Cambridge, CB3 OHA, UK

${ }^{5}$ APC, AstroParticule et Cosmologie, Université Paris Diderot, CNRS/IN2P3, CEA/lrfu, Observatoire de Paris, Sorbonne Paris Cité, 10, rue Alice Domon et Léonie Duquet, 75205 Paris Cedex 13, France

${ }^{6}$ Computational Cosmology Center, Lawrence Berkeley National Laboratory, Berkeley, California, U.S.A.

${ }^{7}$ Space Sciences Laboratory, University of California, Berkeley, CA, 94720, USA

${ }^{8}$ Dipartimento di Fisica, Università di Roma La Sapienza, P.le A. Moro 2, 00185 Roma, Italy

${ }^{9}$ Dipartimento di Fisica, Università di Roma Tor Vergata, Via della Ricerca Scientifica 1, I-00133, Roma, Italy

${ }^{10}$ INFN, Sezione di Tor Vergata, Via della Ricerca Scientifica 1, I-00133, Roma, Italy

${ }^{11}$ Institut d' Astrophysique de Paris (UMR7095: CNRS \& UPMC-Sorbonne Universities), F-75014, Paris, France

${ }^{12}$ INAF/IASF Bologna, via Gobetti 101, I-40129 Bologna, Italy

${ }^{13}$ Dipartimento di Fisica, Università degli Studi di Milano, Via Celoria, 16, Milano, Italy

${ }^{14}$ INAF/IASF Milano, Via E. Bassini 15, Milano, Italy

${ }^{15}$ Laboratoire de l'Accélérateur Linéaire, Univ. Paris-Sud, CNRS/IN2P3, Université Paris-Saclay, Orsay, France

${ }^{16}$ Department of Physics, Gustaf Hällströmin katu 2a, University of Helsinki, Helsinki, Finland

${ }^{17}$ Helsinki Institute of Physics, Gustaf Hällströmin katu 2, University of Helsinki, Helsinki, Finland

${ }^{18}$ Department of Experimental Physics, Maynooth University, Maynooth, Co. Kildare, W23 F2H6, Ireland

${ }^{19}$ INFN, Sezione di Roma, P.le Aldo Moro 5, I-00185, Roma, Italy

${ }^{20}$ Agenzia Spaziale Italiana Science Data Center, Via del Politecnico snc, 00133, Roma, Italy

${ }^{21}$ INAF - Osservatorio Astronomico di Roma, via di Frascati 33, Monte Porzio Catone, Italy

${ }^{22}$ Instituut-Lorentz for Theoretical Physics, Universiteit Leiden, 2333 CA, Leiden, The Netherlands

${ }^{23}$ Department of Theoretical Physics, University of the Basque Country UPV/EHU, 48040 Bilbao, Spain

${ }^{24}$ School of Physics and Astronomy, Cardiff University, The Parade, Cardiff CF24 3AA, UK

${ }^{25}$ Institute of Astronomy, University of Cambridge, Madingley Road, Cambridge, CB3 0HA, UK

${ }^{26}$ SISSA, Via Bonomea 265, 34136, Trieste, Italy

${ }^{27}$ INFN, Sezione di Trieste, Via Valerio 2, I - 34127 Trieste, Italy

${ }^{28}$ DIFA, Dipartimento di Fisica e Astronomia, Universitá di Bologna, Viale Berti Pichat, 6/2, I-40127 Bologna, Italy

${ }^{29}$ INFN, Sezione di Bologna, Via Irnerio 46, I-40127 Bologna, Italy

${ }^{30}$ Université de Toulouse, UPS-OMP, IRAP, F-31028 Toulouse cedex 4, France

${ }^{31}$ CNRS, IRAP, 9 Av. colonel Roche, BP 44346, F-31028 Toulouse cedex 4, France 
${ }^{32}$ Dipartimento di Fisica e Astronomia 'Galileo Galilei', Università degli Studi di Padova, Via Marzolo 8, I-35131, Padova, Italy

${ }^{33}$ INFN, Sezione di Padova, Via Marzolo 8, I-35131 Padova, Italy

${ }^{34}$ INAF-Osservatorio Astronomico di Padova, Vicolo dell'Osservatorio 5, I-35122 Padova, Italy

${ }^{35}$ Department of Physics, Amrita School of Arts \& Sciences, Amritapuri, Amrita Vishwa Vidyapeetham, Amrita University, Kerala 690525, India

${ }^{36}$ SRON (Netherlands Institute for Space Research), Sorbonnelaan 2, 3584 CA Utrecht, The Netherlands

${ }^{37}$ Terahertz Sensing Group, Delft University of Technology, Mekelweg 1, 2628 CD Delft, The Netherlands

${ }^{38}$ DAMTP, Centre for Mathematical Sciences, University of Cambridge, Wilberforce Road, Cambridge, CB3 0WA, UK

${ }^{39}$ Jodrell Bank Centre for Astrophysics, Alan Turing Building, School of Physics and Astronomy, The University of Manchester, Oxford Road, Manchester, M13 9PL, U.K.

${ }^{40}$ Department of Physics \& Astronomy, Tufts University, 574 Boston Avenue, Medford, MA, USA

${ }^{41}$ Institut d'Astrophysique Spatiale, CNRS, UMR 8617, Université Paris-Sud 11, Bâtiment 121, 91405 Orsay, France

${ }^{42}$ Institute for Theoretical Particle Physics and Cosmology (TTK), RWTH Aachen University, D52056 Aachen, Germany.

${ }^{43}$ CAS Key Laboratory for Research in Galaxies and Cosmology, Department of Astronomy, University of Science and Technology of China, Hefei, Anhui 230026, China

${ }^{44}$ Institut Néel, CNRS and Université Grenoble Alpes, F-38042 Grenoble, France

${ }^{45}$ Institute of Astrophysics and Space Sciences, University of Lisbon, Tapada da Ajuda, 1349-018 Lisbon, Portugal

${ }^{46}$ Istituto di Fotonica e Nanotecnologie - CNR, Via Cineto Romano 42, I-00156 Roma, Italy

${ }^{47}$ STFC - RAL Space - Rutherford Appleton Laboratory, OX11 0QX Harwell Oxford, UK

${ }^{48}$ Sorbonne Universités, Institut Lagrange de Paris (ILP), F-75014, Paris, France

${ }^{49}$ IFCA, Instituto de Física de Cantabria (UC-CSIC), Av. de Los Castros s/n, 39005 Santander, Spain

${ }^{50}$ Institut Lagrange, LPNHE, Place Jussieu 4, 75005 Paris, France.

${ }^{51}$ Center for Computational Astrophysics, 160 5th Avenue, New York, NY 10010, USA

${ }^{52}$ Instituto de Astrofísica de Canarias, C/Vía Láctea s/n, La Laguna, Tenerife, Spain

${ }^{53}$ Departamento de Astrofísica, Universidad de La Laguna (ULL), La Laguna, Tenerife, 38206 Spain

${ }^{54}$ The Oskar Klein Centre for Cosmoparticle Physics, Department of Physics, Stockholm University, AlbaNova, SE-106 91 Stockholm, Sweden

${ }^{55}$ The Nordic Institute for Theoretical Physics (NORDITA), Roslagstullsbacken 23, SE-106 91 Stockholm, Sweden

${ }^{56}$ Departamento de Física, Universidad de Oviedo, C. Calvo Sotelo s/n, 33007 Oviedo, Spain

${ }^{57}$ Faculty of Physics, Ludwig-Maximilians Universität, Scheinerstrasse 1, D-81679 Munich, Germany

${ }^{58}$ Excellence Cluster Universe, Boltzmannstr. 2, D-85748 Garching, Germany

${ }^{59}$ School of Physics and Astronomy and Minnesota Institute for Astrophysics, University of Minnesota/Twin Cities, USA

${ }^{60}$ Centro de Estudios de Física del Cosmos de Aragón (CEFCA), Plaza San Juan, 1, planta 2, E44001, Teruel, Spain 
${ }^{61}$ Mullard Space Science Laboratory, University College London, Holmbury St Mary, Dorking, Surrey RH5 6NT, UK

${ }^{62}$ Département de Physique Théorique and Center for Astroparticle Physics, Université de Genève, 24 quai Ansermet, $\mathrm{CH}-1211$ Genève 4, Switzerland

${ }^{63}$ Department of Physics and Astronomy, University of Sussex, Falmer, Brighton, BN1 9QH, UK

${ }^{64}$ European Space Agency, ESAC, Planck Science Office, Camino bajo del Castillo, s/n, Urbanización Villafranca del Castillo, Villanueva de la Cañada, Madrid, Spain

${ }^{65}$ Centro de Astrofísica da Universidade do Porto and IA-Porto, Rua das Estrelas, 4150-762 Porto, Portugal

${ }^{66}$ CEA Saclay, DRF/Irfu/SPP, 91191 Gif-sur-Yvette Cedex, France

${ }^{67}$ Departamento de Física Quàntica i Astrofísica i Institut de Ciències del Cosmos, Universitat de Barcelona, Martíi Franquès 1, 08028 Barcelona, Spain

${ }^{68}$ National Center for Nuclear Research, ul. Hoża 69, 00-681 Warsaw, Poland, and The Astronomical Observatory of the Jagiellonian University, ul. Orla 171, 30-244 Kraków, Poland

${ }^{69}$ LAPTh, Université Savoie Mont Blanc \& CNRS, BP 110, F-74941 Annecy-le-Vieux Cedex, France

${ }^{70}$ Instituto de Física, Universidade Federal do Rio de Janeiro, 21941-972, Rio de Janeiro, Brazil

${ }^{71}$ Observatório do Valongo, Universidade Federal do Rio de Janeiro, Ladeira Pedro Antônio 43, 20080-090, Rio de Janeiro, Brazil

${ }^{72}$ LPNHE, CNRS-IN2P3 and Universités Paris 6 \& 7, 4 place Jussieu F-75252 Paris, Cedex 05, France

${ }^{73}$ Department of Astronomy and Space Science, Sejong University, Seoul 143-747, Korea

${ }^{74}$ INFN, Sezione di Pisa, Largo Bruno Pontecorvo 2, 56127 Pisa, Italy

${ }^{75}$ SRON (Netherlands Institute for Space Research), Sorbonnelaan 2, 3584 CA Utrecht, The Netherlands

${ }^{76}$ Terahertz Sensing Group, Delft University of Technology, Mekelweg 1, 2628 CD Delft, The Netherlands

${ }^{77}$ Laboratoire de Physique Théorique (UMR 8627), CNRS, Université Paris-Sud, Université Paris Saclay, Bâtiment 210, 91405 Orsay Cedex, France

${ }^{78}$ Institute of Cosmology and Gravitation, University of Portsmouth, Dennis Sciama Building, Burnaby Road, Portsmouth PO1 3FX, United Kingdom

${ }^{79}$ Dipartimento di Fisica, Università di Milano Bicocca, Milano, Italy

${ }^{80}$ INFN, sezione di Milano Bicocca, Milano, Italy

E-mail: paolo.natoli@unife.it

Abstract. We present an analysis of the main systematic effects that could impact the measurement of CMB polarization with the proposed CORE space mission. We employ timeline-to-map simulations to verify that the CORE instrumental set-up and scanning strategy allow us to measure sky polarization to a level of accuracy adequate to the mission science goals. We also show how the CORE observations can be processed to mitigate the level of contamination by potentially worrying systematics, including intensity-to-polarization leakage due to bandpass mismatch, asymmetric main beams, pointing errors and correlated noise. We use analysis techniques that are well validated on data from current missions such as Planck to demonstrate how the residual contamination of the measurements by these effects can be brought to a level low enough not to hamper the scientific capability of the mission, nor significantly increase the overall error budget. We also present a prototype of the CORE photometric calibration pipeline, based on that used for Planck, and discuss its robustness 
to systematics, showing how CORE can achieve its calibration requirements. While a fine-grained assessment of the impact of systematics requires a level of knowledge of the system that can only be achieved in a future study phase, the analysis presented here strongly suggests that the main areas of concern for the CORE mission can be addressed using existing knowledge, techniques and algorithms.

ArXiv ePrint: 


\section{Contents}

1 Introduction 1

2 Map-making for CMB experiments 3

3 Simulations 4

4 Analysis of simulated noise maps 6

4.1 Baseline scanning strategy 9

4.2 Optimizing the scanning strategy 11

$4.31 / f$ noise performance 13

5 Cross-correlated noise $\quad 14$

6 Bandpass mismatch $\quad 16$

6.1 Model of the bandpass mismatch effect 18

6.2 Simulations of the bandpass mismatch effect 19

$\begin{array}{ll}6.3 \text { Correction algorithm } & 19\end{array}$

7 Asymmetric beam $\quad 21$

7.1 Real space convolution and first-order de-projection 23

$\begin{array}{lll}7.2 & \text { Harmonic space } & 26\end{array}$

$\begin{array}{lll}7.3 & \text { Beam asymmetry conclusions } & 27\end{array}$

8 Calibration $\quad 28$

8.1 Time dependence of the dipole signal 30

$\begin{array}{lll}8.2 & \text { Systematics } & 31\end{array}$

8.3 Systematics due to the Galaxy 33

9 Pointing accuracy and reconstruction uncertainty 35

10 Conclusions 36

\section{Introduction}

The Standard Model of Cosmology owes its emergence to increasingly accurate observations as much as to theoretical advancement. As new experiments are designed and deployed, the quest for precision and accuracy is becoming more demanding than ever. In the field of cosmic microwave background (CMB) observations, the forefront of research has shifted in recent years from the temperature anisotropies to polarization, a much weaker signal, which has increased scientific expectations and concerns about the analysis. Accurate measurements of CMB polarization pose significant challenges to observational strategies (de Bernardis et al. 2017) as well as to the analysis of data. The Planck results have set a milestone by reaching a level where systematic errors, arising either in the instrumental chain or from contamination by spurious emission, surpass those from stochastic noise in the detectors, both for the CMB temperature power spectrum (Planck Collaboration et al. 2014a, 2016h) and for polarization on large angular scales (Planck Collaboration et al. 2016g,h). The error budget 
of future experiments, whose focal plane arrays will contain thousands of polarization sensitive detectors, will be dominated by systematics even for small scale polarization. It is therefore critical to ensure that these contaminants can be controlled to a level that does not jeopardize the science goals of the mission.

The impact of systematic effects plays a central role in the analysis of modern CMB experiments (Baxter et al. 2015; Bennett et al. 2013; BICEP2 Collaboration et al. 2016; Louis et al. 2016; Planck Collaboration et al. 2016c,f) and is the main subject of several papers as well (e.g., Griffiths and Lineweaver (2004); Karakci et al. (2013); Miller et al. (2009a); Planck Collaboration et al. (2016d)), many of them focusing specifically on polarization specific systematics and their treatment (Kaplan and Delabrouille 2002; Miller et al. 2009b; Pagano et al. 2009; Shimon et al. 2008). The definition of a systematic effect is somewhat dependent on the context. Strictly speaking, any contamination which is not the signal of interest and does not exhibit a purely stochastic behavior may be regarded as a systematic. The CMB community has traditionally used the term in a wider sense, considering any contamination that deviates from ideal, white noise as a systemetic. In this sense, long time scale (i.e., correlated or ' $1 / f$ ') noise may be considered as a systematic contribution, while being from another point of view a purely random component with a zero expectation value.

This paper is part of a set describing the scientific performance of the proposed CORE satellite, which is designed to map CMB polarization to an accuracy only limited by cosmic variance over a broad range of scales. It explores several aspects related to the expected quality of CORE's polarization measurements. We employ a realistic simulation pipeline to produce time ordered data for a year's worth of observations, which we then reduce to maps of intensity and polarization using a state of the art map-making code. We analyse these maps to assess the overall quality of the CORE full sky polarization measurements, in view of the proposed scanning strategy and instrumental design. We include in the simulations a number of realistic effects that may impact the accuracy of the observations, and show that they are either under control or can be kept under control by employing analysis techniques already used by the CMB community. The approach we follow consists in studying one effect at a time, which allows us to evaluate each contribution in isolation and carefully assess its impact. The obvious drawback is that we may miss potential interactions between different effects, a situation that may be addressed by employing full end-to-end simulations (see, e.g., Planck Collaboration et al. (2016e)). We defer this very demanding analysis to future studies.

The plan of this paper is as follows. We provide in Sect. 2 a brief introduction to the CMB mapmaking methodology, which we use throughout this work. In Sect. 3 we describe the timeline-to-map simulation engine that was used in this work, based on the publicly available TOAST software package. We produce noise-only maps based on a realistic noise model, which are analyzed in Sect. 4 to infer results about the purity of the Stokes parameter maps and show how polarization can be resolved by modulating observations using only the scanning strategy, as opposed to adopting specific hardware such as a rotating half wave plate. In this Section, we also explore possible ways of optimizing the scanning strategy and study the noise properties of detectors in several positions in the focal plane. In Sect. 5 we address the case of dealing with noise that is correlated between detectors. We begin addressing signal related simulations with Sect. 6 where we show how a bandpass mismatch between detectors can be effectively mitigated for CORE. Temperature-to-polarization leakage arising from beam non-idealities is discussed in Sect. 7 where we present correction schemes to be applied either in making the map or in harmonic space afterwards, the latter being supported by a specific semi-analytical approach whose performance is compared to simulations. Sect. 8 presents a prototype in-flight calibration pipeline for CORE and discusses its robustness to selected systematic contamination. We discuss in Sect. 9 the impact of effects not considered earlier and finally draw our conclusions in Sect. 10. 


\section{Map-making for CMB experiments}

This paper deals extensively with the propagation of CORE simulated data from time-ordered observations (also called 'timelines') to maps of the sky. To provide some context, we briefly review map-making algorithms for CMB experiments. We begin by considering a simple model, which only accounts for ideal sky signal and stochastic instrumental noise, and discuss the standard approaches and their computational implications. This model will be elaborated in the following sections to include systematics contributions and to discuss specific procedures to mitigate their impact.

Map-making deals with estimation of maps from timelines containing redundant observations of the sky. This subject has closely followed experimental progress in the field. Map-making schemes devised for COBE (Lineweaver et al. 1994), whose differential measuring technique proved effective in reducing correlated noise, were extended to maps containing millions of pixels for WMAP (Wright et al. 1996). More recent CMB experiments (including Planck) adopt a direct measurement scheme, as opposed to a differential one, in order to gain sensitivity and reduce the complexity of the optical system. This approach, also adopted for CORE, faces higher levels of $1 / f$ noise, which has to be kept under control by employing suitable analysis methods (see, e.g., de Gasperis et al. (2005); Doré et al. (2001); Natoli et al. (2001); Patanchon et al. (2008); Stompor et al. (2002); Tristram et al. (2011))

A widely employed model assumes that the timeline $\mathbf{d}$ depends linearly on the map $\mathbf{m}$ by means of a 'pointing' operator A:

$$
\mathbf{d}=\mathbf{A} \mathbf{m}+\mathbf{n},
$$

where the time-ordered vector $\mathbf{n}$ is a stochastic noise component with zero mean and (usually nondiagonal) covariance matrix $N_{t t^{\prime}} \equiv\left\langle n_{t} n_{t^{\prime}}\right\rangle$ ( $t$ labels time samples) and the vector $\mathbf{m}$ is a discretized image of the $\mathrm{sky}^{1}$, containing maps of the Stokes parameters for intensity $I$ and linear polarization $Q$ and $U^{2}$. The simplest possible model for $\mathbf{A}$ is the so-called 'pencil beam' approximation, which ignores the convolution of the signal by the instrumental beam. In this limit, the projection from the sky to the timeline of Eq. 2.1 reads:

$$
d_{t}=I+Q \cos 2 \psi_{t}+U \sin 2 \psi_{t}+n_{t}
$$

where $(I, Q, U)$ are the value of the Stokes parameters of the sky for a given instrumental pointing and $\psi_{t}$ is the instantaneous detector orientation with respect to a chosen celestial frame. Hence, the pencil beam pointing matrix has only three non-zero entries in each row, equal to $\left[1, \cos 2 \psi_{t}, \sin 2 \psi_{t}\right]$. If the instrumental beam is azimuthally symmetric, the pointing and beam convolution operations commute. If this is the case, we may retain the pencil beam approximation and look for an estimate of the beam-convolved map. On the other hand, if the beam is asymmetric the model 2.2 leads to a biased estimate of the map unless proper treatment is included. This situation is addressed in Section 7 below.

An estimate of the map, $\widetilde{\mathbf{m}}$, can be obtained by applying the generalized least squares (GLS) procedure to Eq. 2.1:

$$
\widetilde{\mathbf{m}}=\left(\mathbf{A}^{T} \mathbf{N}^{-1} \mathbf{A}\right)^{-1} \mathbf{A}^{T} \mathbf{N}^{-1} \mathbf{d},
$$

where $\mathbf{A}^{T}$ denotes the transpose of the pointing operator. The quantity $\left(\mathbf{A}^{T} \mathbf{N}^{-1} \mathbf{A}\right)^{-1}$ is the covariance matrix of $\widetilde{\mathbf{m}}$. The GLS estimate enjoys a number of desirable properties: provided the noise matrix $\mathbf{N}$

\footnotetext{
${ }^{1}$ We shall employ the HEALPix pixelization scheme in what follows (Górski et al. 2005).

${ }^{2}$ Circular polarization $V$ is seldom considered for $\mathrm{CMB}$, since it cannot be produced by Thomson scattering over electrons by an unpolarized and anisotropic radiation field (Kosowsky 1999). Instruments employed for CMB measurements do not normally possess the capability to measure circular polarization.
} 
is correct (in practice, it must be estimated from the data) it is the minimum variance estimator. Furthermore, if the noise is drawn from a multivariate Gaussian distribution, the GLS estimate becomes the maximum likelihood solution. It is, however, intractable to compute the matrix for a real world situation with trillions of time samples and millions of map pixels. The problem can be effectively solved by resorting to iterative techniques, typically employing a conjugate gradient solver (Natoli et al. 2001).

For the moment, we restrict the model to a single detector. Multiple detector maps can be trivially accounted for in the absence of noise that is correlated between detectors. If this is not the case, an optimal solution can still be obtained by taking the correlations in account. We discuss an application to CORE of this scenario is Section 5 below.

In the case of large datasets, it may be desirable to further reduce the computational burden. This can be achieved by using approximate versions of Eq. 2.3. A straightforward way to obtain such an approximation is to model the correlated component of the noise using a set of basis functions (typically piecewise constant offsets of given constant length, although more complicated bases can be used) superimposed on white noise. The problem then reduces to finding a suitable estimate of the coefficients of the basis functions. This class of map-making codes is called destripers (see, for example, Burigana et al. 1999; Delabrouille 1998; Keihänen et al. 2004, 2005; Kurki-Suonio et al. 2009; Maino et al. 1999). Sophisticated implementations of these algorithms can produce results which are statistically indistinguishable from GLS map-making while requiring significantly less computational resources. In this scenario, prior information on the correlated noise properties may be needed (Kurki-Suonio et al. 2009). The most desirable feature of destriping algorithms is that they can be tuned to the desired precision while still controlling their computational cost. The latter of course scales unfavourably with precision, but in real-world applications an advantageous compromise can be usually found by tweaking the offset length. In the following we will make extensive use of a public domain implementation of a generalized destriper, MADAM (Keihänen et al. 2005, 2010).

\section{Simulations}

Simulations play a number of critical roles in CMB missions:

1. Optimization of the design of the mission (both the instrument and the observation) to ensure that the dataset obtained will be sufficient to meet the science goals;

2. Validation and verification of the data analysis pipeline to ensure that the science can be extracted from the mission dataset;

3. Uncertainty quantification and debiasing of the data analysis results using Monte Carlo methods in lieu of the computationally intractable full data covariance matrix.

4. Encapsulation of knowledge on the data taking and processing, allowing e.g. for novel analyses outside of the team.

All of these require a joint simulation and analysis pipeline capable of generating a detailed realization of the full mission dataset and reducing it to the science results; figure 1 provides a schematic overview of such a pipeline. The model of the mission includes both the instrument (including the detector properties, focal plane layout and optical path) and the observations (including the scanning strategy and data-flagging), while the sky model includes the CMB together with all foregrounds (and their impact on the CMB through lensing and scattering). The data simulation operator then applies the mission model to the sky model to generate synthetic time-domain data. The steps of the 
analysis pipeline alternate between mitigation of the systematic effects in the current data domain (pre-processing, component separation, post-processing) and reduction of the statistical uncertainties by transforming the data to a new domain with higher signal-to-noise (map-making, power spectrum estimation). The map- and spectral-domain products are then used to constrain the parameters of any given model of cosmology and fundamental physics, typically in conjunction with other cosmological datasets. Finally the various data representations can be used to provide feedback to refine the mission and sky models.

In this work we particularly focus on the simulation and mitigation of systematic effects to address all three questions, the optimization of the mission design, the validation and verification of the mitigation algorithms and implementations, and the quantification of the residuals after mitigation and their impact on the science results.

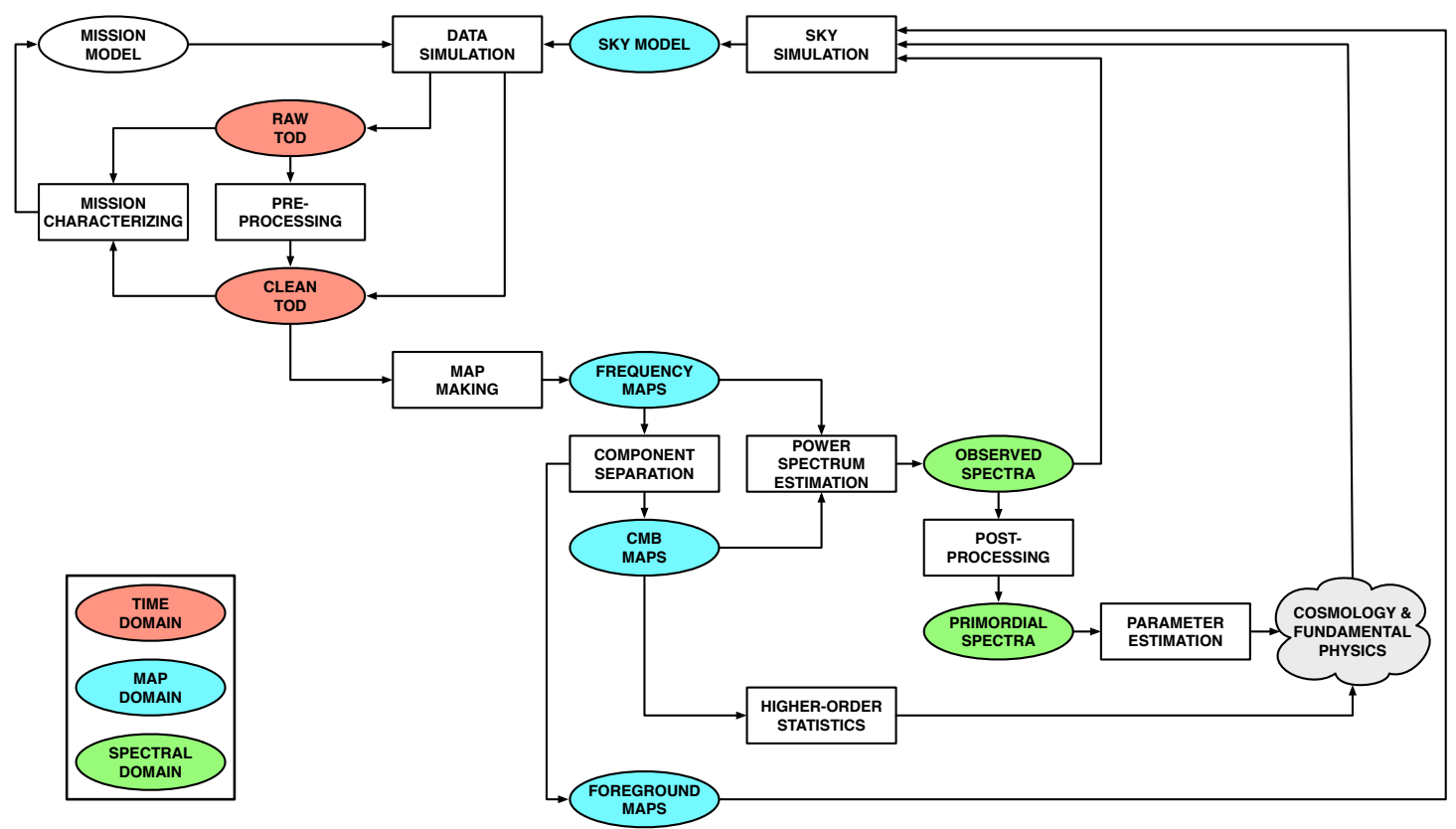

Figure 1. A schematic CMB simulation and analysis pipeline, with rectangular operators acting on oval data objects, which may be time samples (red), map pixels (blue) or spectral multipoles (green). Note the many loops, implying iterative processing.

In the absence of the explicit data covariance matrix, the most computationally challenging elements of this pipeline are those that manipulate the full time-domain data, and in particular the generation and analysis of Monte Carlo realizations used in lieu of this matrix for uncertainty quantification and debiasing. Given the volume of data to be processed, we require highly optimized massively parallel implementations of the simulation, pre-processing and map-making algorithms and significant high performance computing resources. Moreover, since data movement - whether between disk and memory or across distributed memory - is expensive, these steps must be tightly-coupled within an overall time-domain data framework. One such framework, developed for the Planck satellite mission (Planck Collaboration et al. 2016e) but with broad applicability for both satellite and suborbital CMB missions, is the Time-Ordered Astrophysics Scalable Tools (TOAST) package ${ }^{3}$.

${ }^{3}$ http://github.com/hpc $4 \mathrm{cmb} /$ toast 
As well as being highly computationally efficient, any such framework must also be readily adaptable, allowing the rapid prototyping of new algorithms. TOAST is therefore implemented as a python wrapper and data management layer into which new modules can easily be dropped, coupled with compiled libraries (both internal and external) which can be called wherever computational efficiency is a limiting factor. TOAST has been extensively validated and verfied, primarily in conjunction with its use in the Planck full focal plane simulations (Planck Collaboration et al. 2016e) but also through simulations of the CORE and LiteBIRD satellite missions, and in stand-alone comparisons with both analytic calculations and other computational tools.

In this work the TOAST framework calls four main libraries, two internal and two external to the TOAST package:

1. the TOAST pointing library, which generates the dense-sampled pointings for each detector from the sparse-sampled satellite boresight pointing.

2. the TOAST noise simulation library, which generates timelines of noise from each detector's piecewise stationary noise power spectral density functions, provided either as a set of arrays of explicit frequency/power pairs, or as the parameters of an analytic function (typically a white noise level and correlated noise knee frequency and spectral index).

3. the libCONVIQT beam convolution library ${ }^{4}$, a TOAST-compatible implementation of the CONVIQT beam convolution algorithm (Prézeau and Reinecke 2010), which generates timelines of sky signals from each detector's full asymmetric beam and pointings and the simulated sky being observed.

4. the libMADAM map-making library ${ }^{5}$, a TOAST-compatible implementation of the MADAM map-making algorithm (Keihänen et al. 2005, 2010), which makes a destriped map of the sky given some set of time-ordered data and pointings, for some set of detectors.

Using $1+2+4$ we generate coverage and noise maps to evaluate scanning strategies and correlated noise performance, while using $1+3+4$ we generate sky signal maps to evaluate the impact of asymmetric beams. In general, the parameters used, and the analyses of the resulting maps, are discussed in detail in the following sections. For consistency though we employ the same MADAM destriping parameters throughout, in particular setting the destriping offset length and the prior on the correlated noise to maximize statistical efficiency.

We have carried out several tests, considering a variety of offset lengths with and without a noise prior. For simulations, where we know the noise properties, it can be taken to be a priori known and exact; for real data it would be necessary to estimate the noise properties from the timeline data, although the accuracy of this estimate does not need to be especially high for typical applications (Natoli et al. 2002). For the CORE scanning strategy and the noise properties described in Table 1, we found that the best MADAM performance is achieved for a offset of $1 \mathrm{~s}$ and using the exact noise prior (i.e. the description provided to the TOAST noise simulation tool).

\section{Analysis of simulated noise maps}

In this section we describe the noise maps produced with MADAM from timelines simulated with the TOAST pipeline described above. We analyse these maps to assess the robustness of the CORE scanning strategy in measuring the sky Stokes parameters with adequate purity. We also explore

\footnotetext{
${ }^{4}$ http://github.com/hpc4cmb/libconviqt

${ }^{5} \mathrm{http}: / /$ github.com/hpc $4 \mathrm{cmb} / \mathrm{libmadam}$
} 


\begin{tabular}{|c|c|c|}
\hline \multicolumn{2}{|l|}{ Parameter } & Value \\
\hline \multicolumn{2}{|c|}{ Precession angle $\left[{ }^{\circ}\right]$} & 30 \\
\hline \multicolumn{2}{|l|}{ Spin angle $\left[{ }^{\circ}\right]$} & 65 \\
\hline \multicolumn{2}{|c|}{ Precession period [days] } & 4 \\
\hline \multicolumn{2}{|c|}{ Spin period $[\mathrm{s}]$} & 120 \\
\hline \multicolumn{2}{|c|}{ Hours of observation per day [h] } & 24 \\
\hline \multicolumn{2}{|c|}{ Length of a single chunk of data [h] } & 24 \\
\hline \multicolumn{2}{|c|}{ Observation duration [days] } & 366 \\
\hline \multicolumn{2}{|c|}{ Number of detectors } & 2 \\
\hline \multicolumn{2}{|c|}{ Frequency [GHz] } & 145 \\
\hline \multicolumn{2}{|c|}{ FWHM [arcmin] } & 7.68 \\
\hline \multicolumn{2}{|c|}{ Sampling rate $[\mathrm{Hz}]$} & 84.97 \\
\hline \multicolumn{2}{|c|}{ Polarization orientation detector $1\left[^{\circ}\right]$} & -22.5 \\
\hline \multicolumn{2}{|c|}{ Polarization orientation detector $2\left[^{\circ}\right]$} & 67.5 \\
\hline \multicolumn{2}{|c|}{ Knee frequency $f_{k}[\mathrm{mHz}]$} & $0,10,20,50$ \\
\hline \multicolumn{2}{|c|}{ Noise slope $\alpha$} & 1.0 \\
\hline \multicolumn{2}{|l|}{ NET $[\mu \mathrm{K} \sqrt{\mathrm{s}}]$} & 52.3 \\
\hline \multirow{2}{*}{$\begin{array}{l}\text { Deviation from } \\
\text { boresight }\left[{ }^{\circ}\right]\end{array}$} & 'high' & +4.7 \\
\hline & 'low’ & -4.7 \\
\hline \multicolumn{2}{|c|}{$\mathrm{N}_{\text {side }}$} & 1024 \\
\hline \multicolumn{2}{|c|}{ Offset length (with noise prior) [s] } & 1.0 \\
\hline
\end{tabular}

Table 1. Parameters supplied to TOAST to generate the baseline simulations. See text for details. The sampling rate is chosen to ensure four samples per beam FWHM.

possible tweaks to the scanning parameters to verify if they lead to increase robustness. Finally, we analyse the properties of the noise maps to find requirements on the detector knee frequency that ensure that residual contributions to the map on large angular scales are kept under control.

In Table 1 we summarize the parameters we selected as input to TOAST to produce the maps we analyse in this Section. More detail about the parameters in this Table and on CORE's scanning strategy is given in de Bernardis et al. (2017)). We consider the CORE baseline scanning strategy with spin and precession angles of $65^{\circ}$ and $30^{\circ}$ respectively, with corresponding periods of $120 \mathrm{~s}$ and 4 days respectively. In this Section we consider timelines containing only instrumental noise. Signal contributions are examined in Sections 6, 7 and 8 below. We simulate an entire year of observations divided into segments of 24 hours. These are then combined to produce the final map. This segment size is a reasonable compromise between the need to capture long timescale features in the noise and the desire to minimize computational and memory requirements. We assume a noise model with power spectrum density:

$$
P(f)=A\left[\left(\frac{f_{k}}{f}\right)^{\alpha}+1\right]
$$

where $f$ is the frequency, $f_{k}$ the knee frequency which we will vary below, $A$ the amplitude and $\alpha$ a slope equal to 1. A reasonable choice for CORE two detector system is $f_{k}=20 \mathrm{mHz}$ and an amplitude corresponding to a NET of $53.2 \mu \mathrm{K} \sqrt{\mathrm{s}}$. The impact of cross-correlation of noise between detectors is discussed in Section 5 below, were we employ, in place of MADAM a dedicated map-making code, ROMA (de Gasperis et al. 2016), capable of taking cross-correlation information in account to deliver a lower noise solution. 

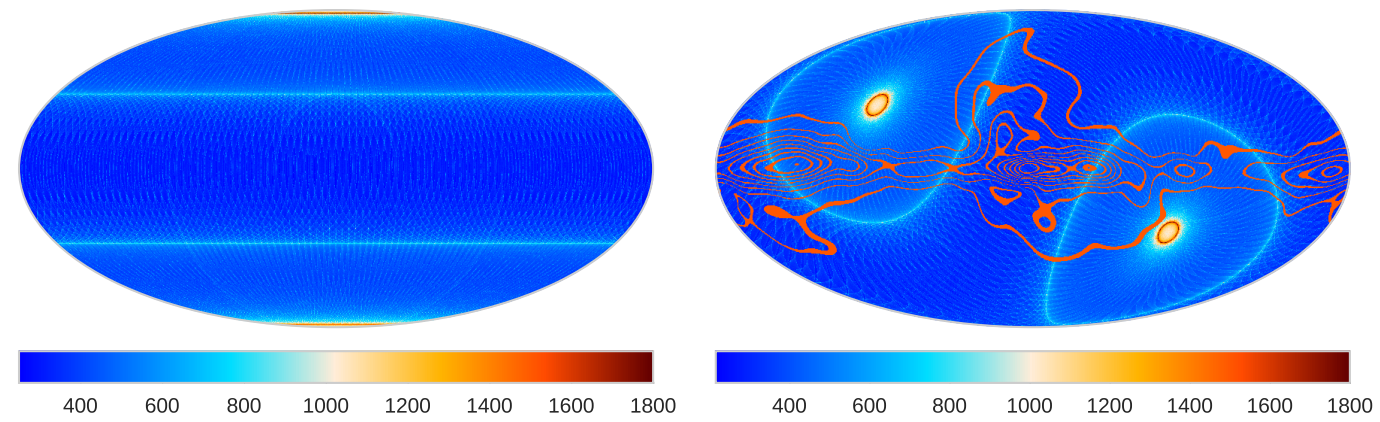

Figure 2. Hit map for a pair of detectors located at the center of the focal plane after one year of observation. The map is shown in Ecliptic coordinates (left) and in Galactic coordinates (right). We also show (orange contours) an estimate of the polarization amplitude of the foregrounds at $70 \mathrm{GHz}$, a frequency close to the minimum emission of diffuse foregrounds. The outermost contour corresponds to $1.3 \mu \mathrm{K}$ in polarized intensity, and the subsequent contours to further steps of $1.3 \mu \mathrm{K}$.

In the remainder of this Section we consider a pair of polarization sensitive detectors at $145 \mathrm{GHz}$ at the same position in the focal plane, either at the boresight or at the edges of the focal plane, oriented at $-22.5^{\circ}$ and $67.5^{\circ}$ with respect to the scan direction. This choice equalizes the noise power in the $Q$ and $U$ Stokes parameters and produces EE and BB angular power spectra with similar amplitude. In any case, any particular choice of orientation becomes irrelevant when producing maps from a large number of detectors, assuming their orientations are evenly spaced. We adopt these particular values for the sole purpose of achieving balance in the Stokes parameters in this minimal two-detector exercise. We also simulate the sky as observed by detectors at the edge of the focal plane. These are modelled by considering two pairs of detectors at $\pm 4.7^{\circ}$ with respect to the boresight along the direction orthogonal to the scan direction. These have the same polarization orientation as the boresight detectors and are labelled as 'high' and 'low' detectors in Table 1 and hereinafter.

The hit map for the two-detector case described above is given in Fig. 2, having chosen a boresight direction. The irregular small-scale features, hardly visible at standard figure size, would be diluted when considering a larger number of detectors. From this simple exercise, we show that the CORE scanning strategy leads to a complete sky coverage in around 6 months, yet a coverage of around $45 \%$ of the full sky is achieved in just 4 days thanks to the wide precession. After one year, all pixels in the sky have been observed at least 200 times by a pair of detectors, assuming 3.4 arcmin pixels (HEALPix $\mathrm{N}_{\text {side }}=1024$ ). The hit map in Galactic coordinates is overplotted with an estimate of the total diffuse polarized foregrounds at $70 \mathrm{GHz}$, where emission is close to a minimum (Planck Collaboration et al. 2016b). This estimate was obtained using the Planck $353 \mathrm{GHz}$ and $30 \mathrm{GHz}$ polarized maps respectively as dust and synchrotron templates. This simple exercise shows how the CORE scanning strategy provides high signal-to-noise sampling of regions that are remarkably clean of polarized foreground emission. Of course, the use of specific component separation techniques will reduce residual foreground emission considerably (Remazeilles et al. 2017).

We compute the white noise covariance matrix for the chosen scanning strategy. This gives a $3 \times 3$ symmetric positive definite matrix for $(I, Q, U)$ in each pixel. In so doing, we ignore $1 / f$ contributions that would generate correlations between pixels. For these $3 \times 3$ matrices we compute the reciprocal condition number $(\mathrm{RCN})$, defined as the ratio of its smallest to largest eigenvalue. The $\mathrm{RCN}$ is an useful indicator to decide whether a matrix is ill-conditioned. We employ it here to verify the purity of the map-making solution for the Stokes parameters. A RCN of $1 / 2$ is achieved only in ideal cases, while values too low, even if still adequate from a purely numerical standpoint, may leave the system vulnerable to non-idealities, by amplifying the effects of systematic contributions in 


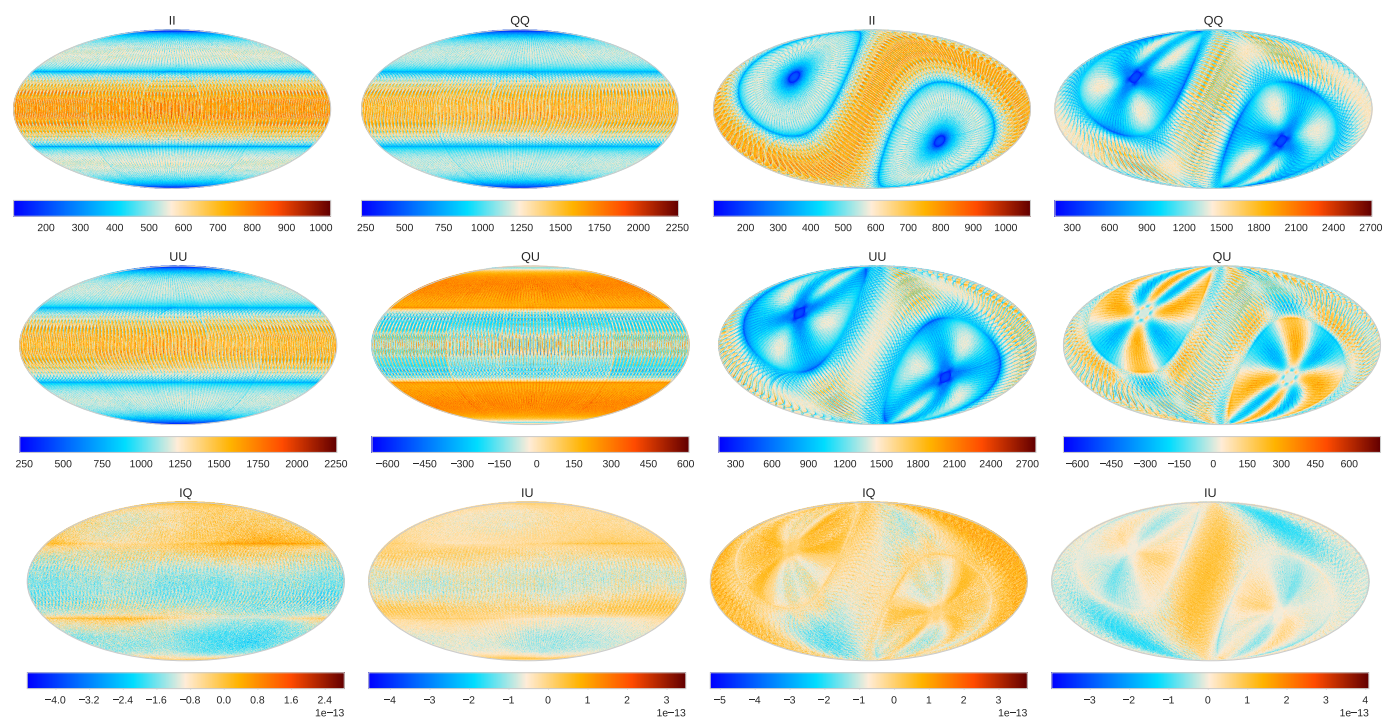

Figure 3. Elements of the white noise covariance matrix for a pair of boresight detectors displayed as maps in units of $\mu K^{2}$ : $I I$ (top left), $Q Q$ (top right), $U U$ (center left), $Q U$ (center right), $I U$ (bottom left), $I Q$ (bottom right). Coordinates are Ecliptic (left columns) and Galactic (right columns). Notice that $I Q$ and $I U$ correlations are very weak.

the timeline. We set a limit of a minimum RCN of $10^{-2}$ for the present analysis. We also compute the angular power spectra (APS) of the simulated noise maps. The noise APS allow to assess the destriping efficiency of MADAM in controlling spurious low-frequency contributions.

\subsection{Baseline scanning strategy}

In Fig. 3 we show maps of the elements of the $3 \times 3$ white noise covariance matrices produced by MADAM for the case of boresight detectors for both Ecliptic and Galactic coordinates, and histograms of these matrix elements are shown in Fig. 4. Larger values of these histograms reflect larger pixel variance of the noise maps. An effective scanning strategy will achieve compact histograms with low mean values and minimal tails. These requirements are reasonably satified by CORE, as observed in Fig. 4: the histograms do not possess large tails, total intensity has smaller values with respect to polarization by a factor of 2, and the $Q Q$ and $U U$ histograms are very similar to one another. This last property is influenced by the particular choice of the orientations of the detectors as explained above. In addition, intensity and polarization show almost negligible correlations, while $Q U$ does show significant correlation features. These however are expected to vanish when a multidetector map from an entire frequency channel is produced, in view of the large number of detectors per frequency expected by CORE, and the desirable variation in mutual orientation (in fact, it would suffice to consider only four detectors with polarization angles at exactly $45^{\circ}$ to each other to have this correlation vanish).

As described above, a useful quantity for assessing the map-making inversion is the RCN. The $\mathrm{RCN}$ for the boresight solution is shown in Fig 5. Reasonable requirements for optimal inversion are an average value across the histogram higher than 0.25 and no pixels with values lower than $10^{-2}$. With the CORE scanning strategy, we obtain an average value of about 0.41 and no pixels with values lower than 0.2, hence the separation of the Stokes parameters as allowed by the scanning strategy alone is very good. This shows that from the point of view of map-making effectiveness, CORE can efficiently modulate polarization without resorting to a rotating half-wave plate. It should be 

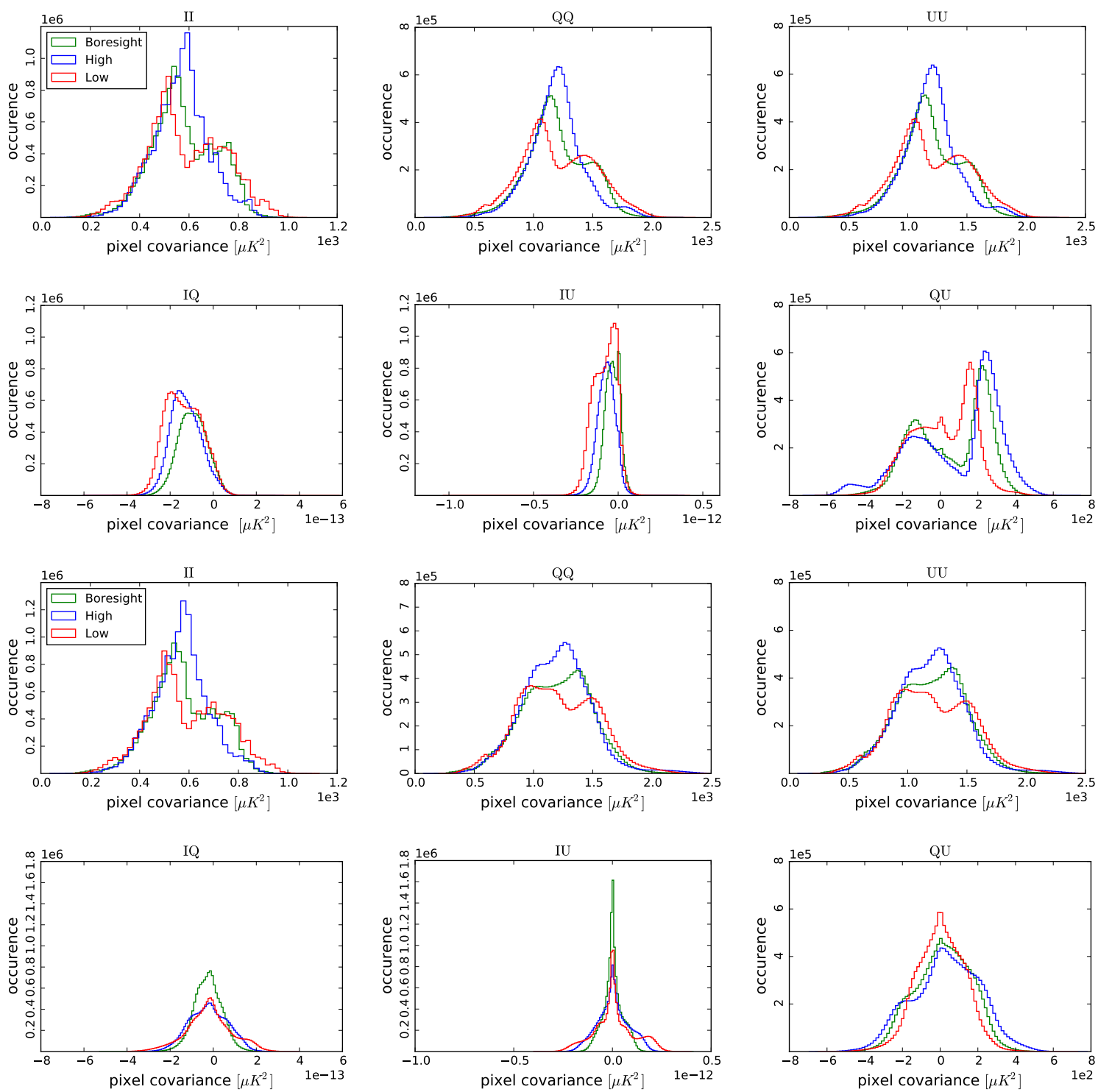

Figure 4. Histograms of the $3 \times 3$ pixel covariance matrix elements in Ecliptic coordinates (first and second rows) and in Galactic coordinates (third and fourth rows). There are minimal intensity-to-polarization couplings (notice the change of scale) but significant $Q U$ residual correlation.

mentioned that we have investigated the ideal performance of the scanning strategy here, neglecting, for example, cross-polar leakage.

In Fig. 4 we also show the histograms of the noise covariance matrix for the high (blue) and low (red) detectors. One of the risks for detectors at the edge of the focal plane is not achieving complete sky coverage. This is avoided by imposing the condition that the sum of the spin and precession angles is more than $90^{\circ}$ for the entire focal plane. In CORE, the sum of these angles for the low detectors is $90.3^{\circ}$ allowing for the complete sky coverage across the whole focal plane. We have used these simulations to verify that this is indeed the case. The histogram shapes are similar to the boresight ones, and there are no anomalous values of the noise covariance matrix elements. In Fig. 5 we show the RCN for the high and low detectors, which on average are quite similar to boresight. 


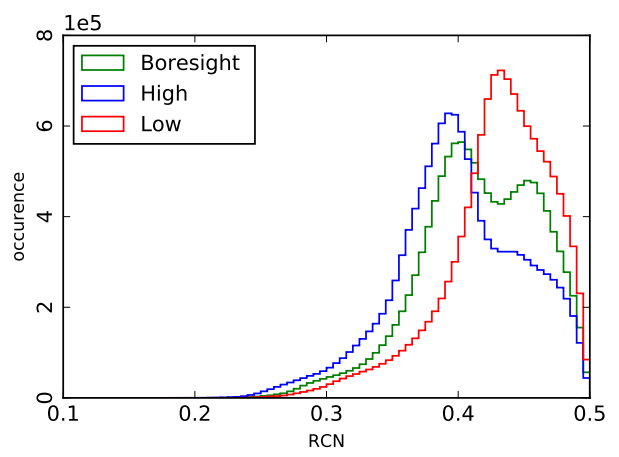

Figure 5. Histograms of the reciprocal condition numbers for the boresight, high and low detectors.
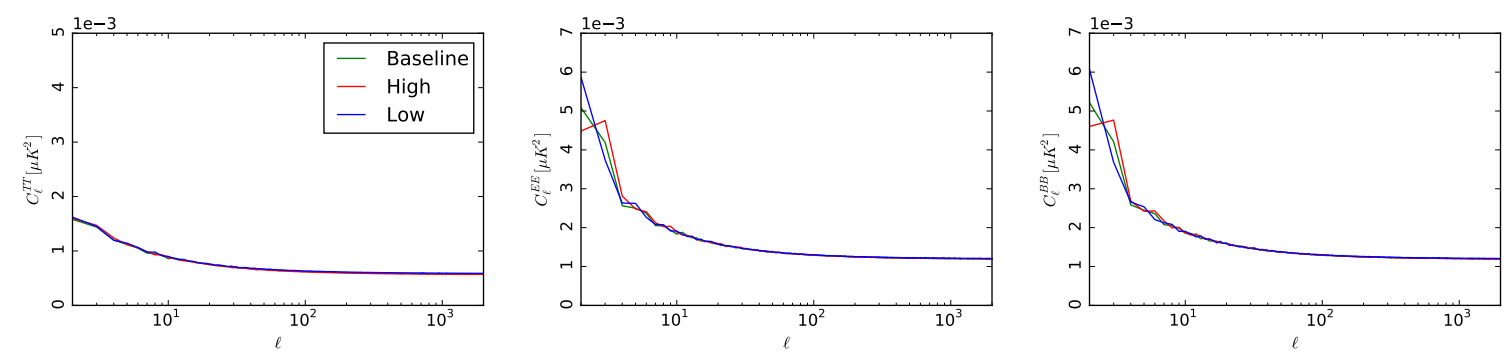

Figure 6. Angular power spectra for $T T$ (left), $E E$ (centre) and $B B$ (right) of the baseline simulations for the boresight, high and low detectors. We show the average of 1000 simulations and the $1 \sigma$ dispersion for the boresight case (shaded regions).

Low detectors show slightly higher RCN, high detectors show slightly lower RCN. This allows us to extend the above conclusions about the clean separation of the Stokes parameters to the whole focal plane.

In Fig. 6 we show the average $T T, E E$ and $B B$ APS from 1000 noise realizations for the boresight, high and low detectors (details of our Monte Carlo pipeline are given in Appendix A). We also show the $1 \sigma$ dispersion of the boresight case. As already noted for the RCN, the APS of different detectors are all similar. The APS of low detectors show slightly lower amplitudes than the other two. The $E E$ and $B B$ amplitudes are practically the same as a result of the choice of the polarization orientations. All spectra show a large scale (low multipole) excess, due to residual $1 / f$ contribution after destriping. The impact of different knee frequencies is discussed in Section 4.3.

\subsection{Optimizing the scanning strategy}

We investigate possible optimizations of the CORE scanning strategy by analysing the effect of varying the spin angle and the precession angle. We consider seven pairs of values keeping the sum of these angles equal to $95^{\circ}$ for the boresight detectors in order to preserve full sky coverage for the entire focal plane. In this way we define seven 'tweaked' cases to be compared to the baseline CORE scanning strategy (see Table 2 for the chosen values, all the other parameters are the same as in Table 1).

In Fig. 7 we show the RCN of the noise covariance matrices for the tweaked cases considering the boresight, high and low detectors. The RCN are all quite similar with average values around 0.4 for all cases. Cases from 1 to 5 show larger tails towards lower RCN values and therefore their 


\begin{tabular}{|l|c|c|c|c|c|c|c|c|}
\hline Parameter & Baseline & Tweak 1 & Tweak 2 & Tweak 3 & Tweak 4 & Tweak 5 & Tweak 6 & Tweak 7 \\
\hline \hline Precession angle $\left[{ }^{\circ}\right.$ ] & 30 & 32 & 34 & 36 & 38 & 40 & 45 & 50 \\
\hline Spin angle $\left[{ }^{\circ}\right]$ & 65 & 63 & 61 & 59 & 57 & 55 & 50 & 45 \\
\hline
\end{tabular}

Table 2. Parameters modified with respect to Table 1 to obtain tweaked cases to evaluate a possible optimization of the CORE scanning strategy. The first column gives the baseline parameters.
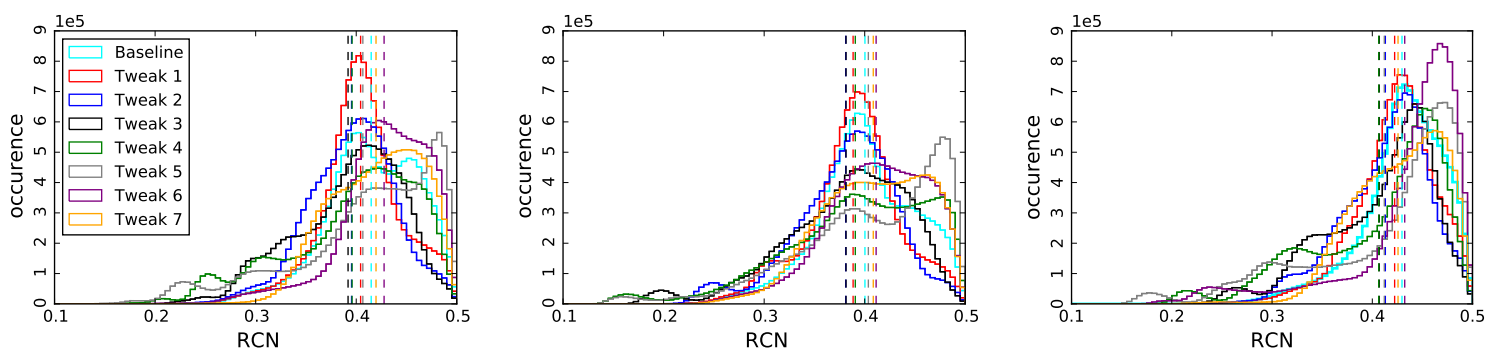

Figure 7. Histograms of the RCN for the boresight (left), high (centre) and low (right) detectors in the tweaked cases compared to the baseline (cyan). The vertical dotted lines show the mean values.
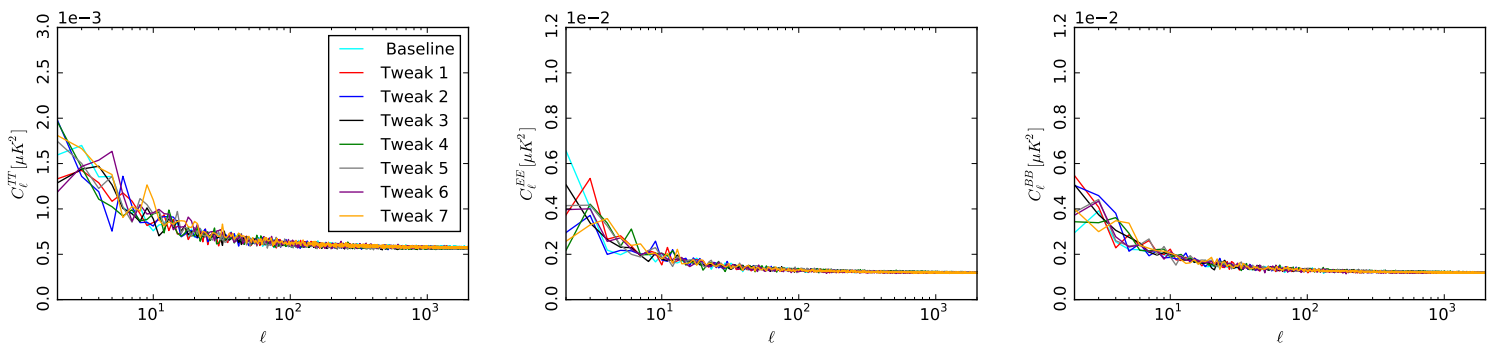

Figure 8. $T T$ (left), $E E$ (centre) and $B B$ (right) APS of the baseline simulations for the boresight detectors compared to the tweaked cases described in Table 2.

average RCN is slightly lower. Cases 6 and 7 show slightly improved RCN with respect to the baseline especially for the boresight detectors. The improvements are less evident when the high and low detectors are considered. The highest mean RCN is achieved by case 6 with a value of about 0.42 for the boresight detector which, given the dispersion of the RCN values shown in Fig. 7, is not significantly different from the 0.41 achieved by the baseline, in view of the generous spread of RCN values. This is a small improvement that would require significant changes in spin and precession angles, and would have negative impacts on other subsystems of the spacecraft (for example, a lower power supply due to the change in solar aspect angle).

In Fig. 8 we show the APS of the noise maps for the boresight, high and low detectors. All the APS here are the result of the average over 10 noise realizations. The APS of the tweaked cases are compared to the baseline and its $1 \sigma$ dispersion delimited by the cyan shaded region. At small scales the APS are all almost identical. Larger differences are evident at large scales, but they are well inside the $1 \sigma$ dispersion.

Our conclusion from this exercise is that any gain in tweaking the scanning strategy parameters is modest and probably is not worth attempting, at least for the figures of merit considered above, but does leave some flexibility to optimize others. 

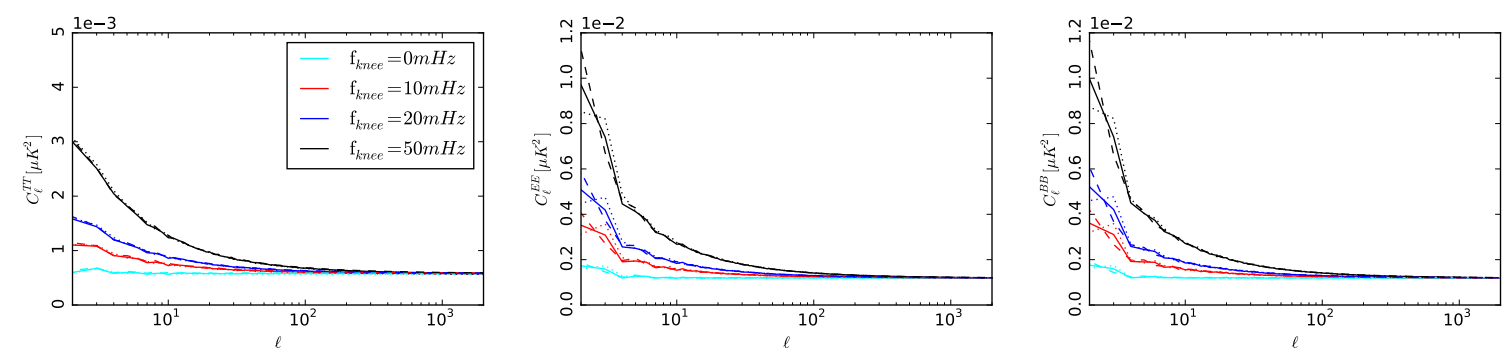

Figure 9. $T T$ (left), $E E$ (centre) and $B B$ (right) APS of the baseline simulations for the boresight detectors considering several knee frequencies $f_{k}$. We show the APS from the boresight detectors (solid lines), high detectors (dotted lines) and low detectors (dashed lines).

\section{3 $1 / f$ noise performance}

In this Section we investigate the effect of the low frequency noise properties. We simulate a year of observations for a pair of boresight, high or low detectors considering several knee frequencies $f_{k}$ in the range between $0-50 \mathrm{mHz}$, a range that appears reasonable in view of CORE's planned detectors. As mentioned above, we make use of a noise prior in MADAM, which requires as input an estimate of the noise power spectral density. We provide here the true underlying power spectrum of the noise. Even if this may be considered an optimistic choice, in practice the impact on the results of a mismatch between the true and estimated noise properties is weak, as noted in Sect. 3 above. We always use $1 \mathrm{~s}$ as the MADAM offset length. We generate 1000 Monte Carlo (MC) realizations (see Appendix A) and apply MADAM to produce noise-only maps. The amplitude of residuals can be turned in a requirement on the maximum acceptable knee frequency.

In Fig. 9 we show the average APS from $1000 \mathrm{MC}$ realizations for the knee frequencies $f_{k}$ of $10 \mathrm{mHz}$ (red line), $20 \mathrm{mHz}$ (blue line), $50 \mathrm{mHz}$ (black line). They are compared with the pure white noise case $f_{k}=0 \mathrm{mHz}$ and its $1 \sigma$ dispersion (cyan line and shaded region). In the same Figure we show the results for a pair of low detectors (dashed lines) and high detectors (dotted lines). As expected, we do not observe any difference between the position of the detectors in the focal plane. The effect of the destriping residuals is a larger amplitude of the noise spectrum at large scales $(\ell<100)$ and, as expected, the residuals increase with increasing $f_{k}$. The $10 \mathrm{mHz}$ case lies at the edge of the $1 \sigma$ dispersion of the white noise MC. Knee frequencies lower than this value will generate noise maps that cannot be practically distinguished from pure white noise both in temperature and polarization. Therefore low frequency noise drifts have negligible effects if $f_{k}<10 \mathrm{mHz}$. We notice that a knee frequency of $20 \mathrm{mHz}$ is still an acceptable compromise showing an increase in the noise APS mostly confined to $\ell<10$.

We now turn to comparing the amplitude of $1 / f$ noise residuals with primordial polarization signals. In the above analysis we considered a pair of detectors at $145 \mathrm{GHz}$. The proposed configuration of CORE has 2100 detectors in the frequency range from 60 to $600 \mathrm{GHz}$ (de Bernardis et al. 2017). We can use the above results to infer the impact in the APS of a noise map obtained from the entire focal plane. We consider the six cosmological channels between 130 and $220 \mathrm{GHz}$ which have the lowest noise, and produce a noise power spectrum from the combination of these channels by inverse noise weighting. We then rescale the amplitude of the noise APS derived from a pair of detectors (shown in Fig. 9) to match this noise spectrum at $\ell=300$. This approach does not fold in contributions to the final error budget arising from sources other than instrumental noise (e.g. foreground separation residuals) and for this reason we avoid using channels below $130 \mathrm{GHz}$ and above $220 \mathrm{GHz}$, which still contain useful CMB signal. The same combination of channels has been con- 


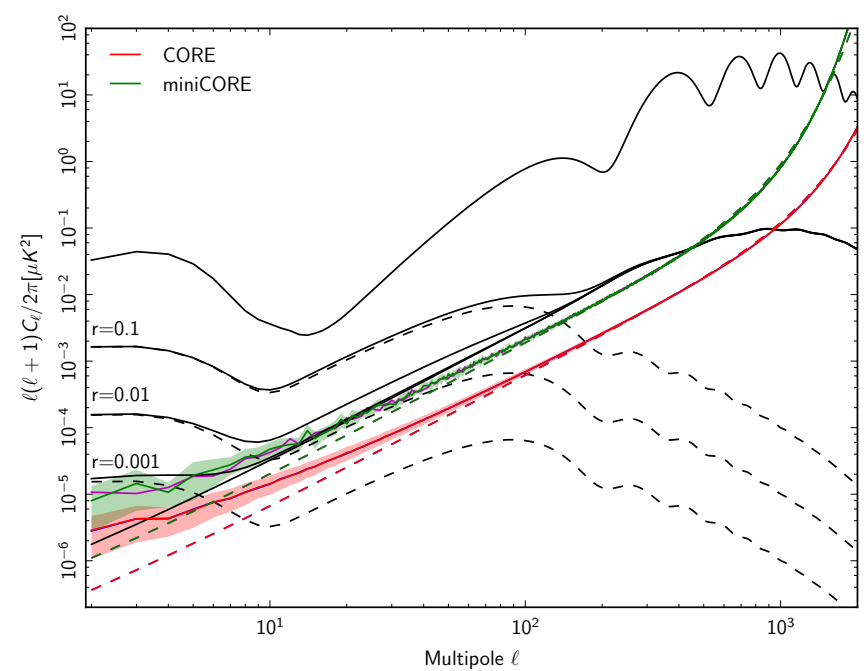

Figure 10. Polarized noise angular power spectra (coloured lines) in $E E$ and $B B$ for $C O R E$ (red and blue lines respectively) and miniCORE (magenta and cyan respectively), considering all channels between 130 and $220 \mathrm{GHz}$, compared to $E E$ and $B B C M B$ theoretical spectra for several values of the tensor-to-scalar ratio $r$ (the solid curve includes lensing B modes). The shading corresponds to the $1 \sigma$ uncertainty region. See text for details.

sidered in the CORE cosmological parameters and inflationary forecasts discussed in Di Valentino et al. (2016) and CORE Collaboration et al. (2016).

Results are shown in Fig. 10, where they are compared to $E E$ and $B B$ CMB spectra, considering for the latter several values of the tensor to scalar ratio $r$, while the other cosmological parameters are kept to the Planck 2015 updated best fit values (Planck Collaboration et al. 2016g). The red dashed line shows the noise spectrum from the inverse noise weighting of the six CMB channels as described above. The solid line shows noise APS of a pair of detectors as result of the average of 1000 noise realizations (assuming $f_{k}=50 \mathrm{mHz}$ and a beam of $5 \mathrm{arcmin}$ ), rescaled to match the dashed line at $\ell=300$. We show both $E E$ (blue line) and $B B$ (red line) spectra although they are almost indistinguishable. We also show the $1 \sigma$ dispersion of the realizations as the shaded region. The effect of $1 / f$ noise is noticeable at $\ell \lesssim 100$. However, even in this pessimistic assumption of $f_{k}=50 \mathrm{mHz}$, the noise spectrum is well below the $B B$ spectrum for $r=10^{-3}$ for $\ell \lesssim 10$. We also show in Fig. 10 the forecasted noise spectra (magenta for $E E$, cyan for $B B$ ) for the so-called miniCORE design, a downscoped configuration of CORE (see de Bernardis et al. (2017) for more detail). We follow the same approach described above, but consider the miniCORE parameters for the beam FHWM (11.9 arcmin at $145 \mathrm{GHz}$, corresponding to a sampling rate of $54.8 \mathrm{~Hz}$ ) and averaging over 10 noise realizations instead of 1000. All the other parameters, except of course the number of detectors per channel, are identical to CORE (see table 1). Despite the noise level for miniCORE is now significantly closer to the $B B$ spectrum for $r=10^{-3}$, this design still allows plenty of margin for an accurate measurement of tensor modes in view of $1 / f$ residual contamination, especially considering that $f_{k}=50 \mathrm{mHz}$ is taken here as a worst case scenario.

\section{Cross-correlated noise}

Cross-correlated noise contributions between different detectors have been reported by several CMB experiments (see e.g. Masi et al. 2006; Patanchon et al. 2008; Planck Collaboration et al. 2016g). They are a source of potential concern for the densely packed focal planes of the current and forth- 
coming generation of CMB experiments. The presence of these common modes is usually neglected during analysis, though optimal treatment can easily be included in the GLS framework discussed in Sect. 2, at the cost of increasing the computational burden (de Gasperis et al. 2016; Patanchon et al. 2008), as detailed in Appendix B. In short, a solution formally identical to Eq. 2.3 can be obtained for the the estimated map $\widetilde{\mathbf{m}}$ :

$$
\widetilde{\mathbf{m}}=\left(\mathbf{A}^{T} \mathbf{N}^{-1} \mathbf{A}\right)^{-1} \mathbf{A}^{T} \mathbf{N}^{-1} \mathbf{d},
$$

where $\mathbf{d}$ and $\mathbf{A}$ are respectively a multi-detector timeline and pointing matrix and $\mathbf{N}$ a generalized noise matrix:

$$
\mathbf{N} \equiv\left\langle\mathbf{n}_{t^{\prime}} \mathbf{n}_{t^{\prime}}\right\rangle=\left(\begin{array}{ccc}
\left\langle n_{t}^{(1)} n_{t^{\prime}}^{(1)}\right\rangle & \cdots & \left\langle n_{t}^{(1)} n_{t^{\prime}}^{(k)}\right\rangle \\
\vdots & \ddots & \vdots \\
\left\langle n_{t}^{(k)} n_{t^{\prime}}^{(1)}\right\rangle & \cdots & \left\langle n_{t}^{(k)} n_{t^{\prime}}^{(k)}\right.
\end{array}\right),
$$

where $\langle\cdot\rangle$ denotes the expectation value and $(\cdot)$ labels different channels. As is customary, we assume stationary noise, implying that the statistical properties of the noise do not change over the mission lifetime. To be specific, when considering the $j$-th and $\ell$-th detectors and the noise elements $n_{t}^{(j)}$ and $n_{t^{\prime}}^{(\ell)}$ at time $t$ and $t^{\prime}$, respectively, we assume that $\left\langle n_{t}^{(j)} n_{t^{\prime}}^{(\ell)}\right\rangle$ only depends on $\left|t-t^{\prime}\right|$. Moreover, we consider that the noise statistical properties are known and completely described by the noise covariance matrix. We assume that the noise auto- and cross-spectra can be estimated either by on-ground instrument calibration or directly from observational data by dark-sky measurements or iterative procedures (see e.g. Prunet et al. 2001).

To find $\widetilde{\mathbf{m}}$, the optimal GLS formula can be solved iteratively using a Fourier based, preconditioned conjugate gradient method as outlined in Sect. 2. Note however that, given the full noise covariance matrix (whose effective size is proportional to $N+N_{c} \times\left(N_{c}-1\right) / 2$, where $N$ is the total number of detectors and $N_{c}$ is the number of with cross-correlated noise), a single iteration of the preconditioned conjugate gradient solver scales linearly with the total number of samples, but quadratically with $N_{c}$.

We set up a CORE test case by generating a noise timeline with the TOAST software assuming the CORE scanning strategy and detector parameter baseline (see Tab. 1), considering the cases of 2 and 4 detectors for 1 year of operations. Following the behaviour of Planck HFI (Planck Collaboration et al. $2016 \mathrm{~g}$ ), we assume that the noise of the $i$-th detector has the following properties:

$$
\begin{aligned}
n_{i} & =\tilde{n}_{i}+n_{c} \\
P_{i}(f) \equiv\left\langle\tilde{n}_{i} \tilde{n}_{i}\right\rangle & =A\left[\left(\frac{f_{k}}{f}\right)+1\right] \\
P_{i j}(f) \equiv\left\langle n_{i} n_{j}\right\rangle=\left\langle n_{c} n_{c}\right\rangle & =A\left[\left(\frac{f_{1}}{f}\right)^{2}+C\right] \text { for } i \neq j
\end{aligned}
$$

where $\tilde{n}_{i}$ and $n_{c}$ refer to the self- and cross-correlated noise component, with knee frequencies $f_{k}=$ $f_{1}=20 \mathrm{mHz}$. We assume $C=0$ and use the NET from Table 1 for the amplitude $A$. We then generate noise maps with:

1. a GLS map-making algorithm that considers only the diagonal terms of the noise covariance matrix that correspond to the auto-correlated $1 / f$ noise (the ROMA MPI-parallel code, see Natoli et al. 2001 and de Gasperis et al. 2005);

2. an optimal GLS map-making algorithm extended to account for all the off-diagonal terms of the noise covariance matrix that correspond to the correlations between detectors (the extended ROMA MPI-parallel code, see de Gasperis et al. 2016). 
For each case above, we generate the noise-only maps of the Stokes parameters $Q$ and $U$. In Fig.11 we show the difference of the noise-only $Q$ and $U$ maps between the cases with and without crosscorrelated noise in the map-making code (for the 2 detector case). These difference maps show stripy structures, suggesting that the inclusion of the cross-correlation properties in the noise model mitigates the residual correlation along the scan. We then compute the r.m.s. values of the maps, noting very slight differences between cases 1 and 2 above (of few parts out of $10^{4}$ ). However, it should be emphasized that the map r.m.s. is not the most appropriate figure of merit here, since the effect of accounting for the noise cross-correlation is limited only to the very large angular scales. An APS analysis is better suited inasmuch as it separates the contribution of different angular scales. We therefore produce 20 Monte Carlo noise-only maps and generate the corresponding APS. We then compare the average spectra for cases 1 and 2 above by calculating their ratio (see Fig.12). We notice that neglecting the noise cross-correlation results in a larger noise amplitude at very large angular scales. This excess is suppressed by accounting for the detector cross-correlation in the map-making: in fact, we have a decrease of the noise spectrum amplitude up to 5-10\% at very low multipoles. Finally, we compare the APS standard errors as computed from the the dispersion of the simulated Monte Carlo maps (see Fig.12). We find that the inclusion of the cross-correlated noise provides smaller error-bars at the largest angular scales (again, up to 5-10\%), while, as expected, we do not find any relevant difference at smaller scales.

Results shown in Fig. 12 correspond to the 2 detector case. Tests on the 4 detector case have shown the same qualitative behaviour: we still find a decrease in both noise spectrum amplitude and standard deviations of 5-10\% after including the noise cross-correlation information in the mapmaking process. We emphasize that the common-mode noise contribution does not easily integrate down with increasing the number of detectors, as opposite to the auto noise component. For this reason, the noise cross-correlation should be carefully accounted for in any polarization experiment with a large number of detectors, and the map-making process is the natural place to deal with it.

We emphasize that the amplitude of this effect is not straightforward to forecast accurately (as it will crucially depend of the instrumental set-up), but we highlight the importance of having a pipeline able to handle it. Indeed, our analysis shows that the effect of the noise cross-correlation is not negligible. Due to the faintness of the B-mode polarization signal, the improvement provided by the extended map-making algorithm may prove crucial for accurate characterization of such contribution at the largest angular scales: accurate modelling of noise at low resolution is an important task to reliably measure the B mode reionization bump, since for some highly efficient estimators devoted to this task noise misestimation may induce bias (Mangilli et al. 2015), contrary to what happens for GLS map-making.

\section{Bandpass mismatch}

Difference in detector bandpasses induces leakage from intensity to polarization for any astrophysical component with a non-CMB spectrum after calibrating the data on the CMB. This arises after differencing measurement from detectors, with slightly different sensitivity to component signals, to estimate the polarization signal. This effect is studied in Hoang et al. (2017), and it has been shown that the amplitude of the leakage is strongly coupled to the single detector cross-linking parameters measuring the uniformity of angle coverage in each pixel. This systematic effect was observed in Planck data (Planck Collaboration et al. 2016g) at the percent level and required correction to avoid biasing the estimation of CMB polarization (Planck Collaboration et al. 2015a). Given the sensitivity of the CORE mission, this effect has to be studied carefully and correction methods must be designed. In this section, we study this effect in the specific case of CORE baseline scanning strat- 
NOISE ONLY DIFFERENCE MAP - Q STOKES

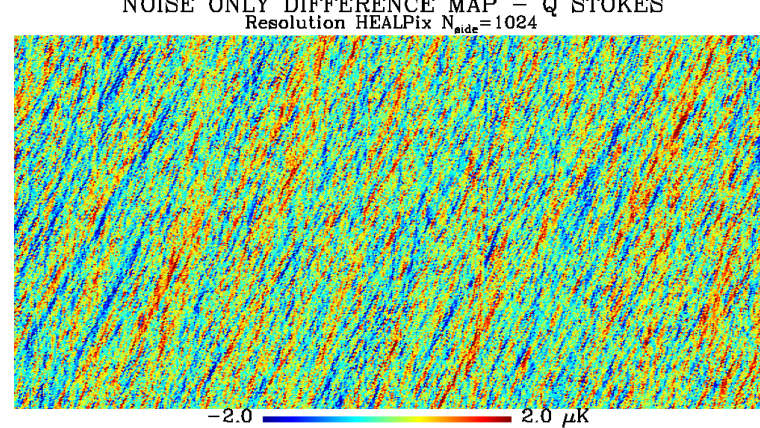

NOISE ONLY DIFFERENCE MAP - U STOKES

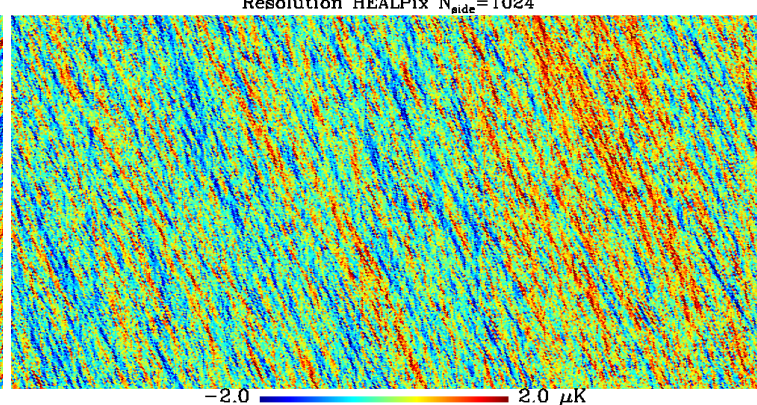

Figure 11. Difference of the noise-only maps of the Stokes parameters $Q$ (left) and $U$ (right) between the cases accounting for and ignoring cross-correlated noise in the map-making (Cartesian projection). Maps have been estimated assuming 2 detectors and one year of operation. Maps are in Ecliptic coordinates, in units of $\mu K$ and at resolution HEALPix $N_{\text {side }}=1024$ (3.435 arcmin/pixel). The stripes are due to the cross-correlated noise, which is mitigated when it is taken into account in the map-making algorithm.

$\mathrm{EE}$

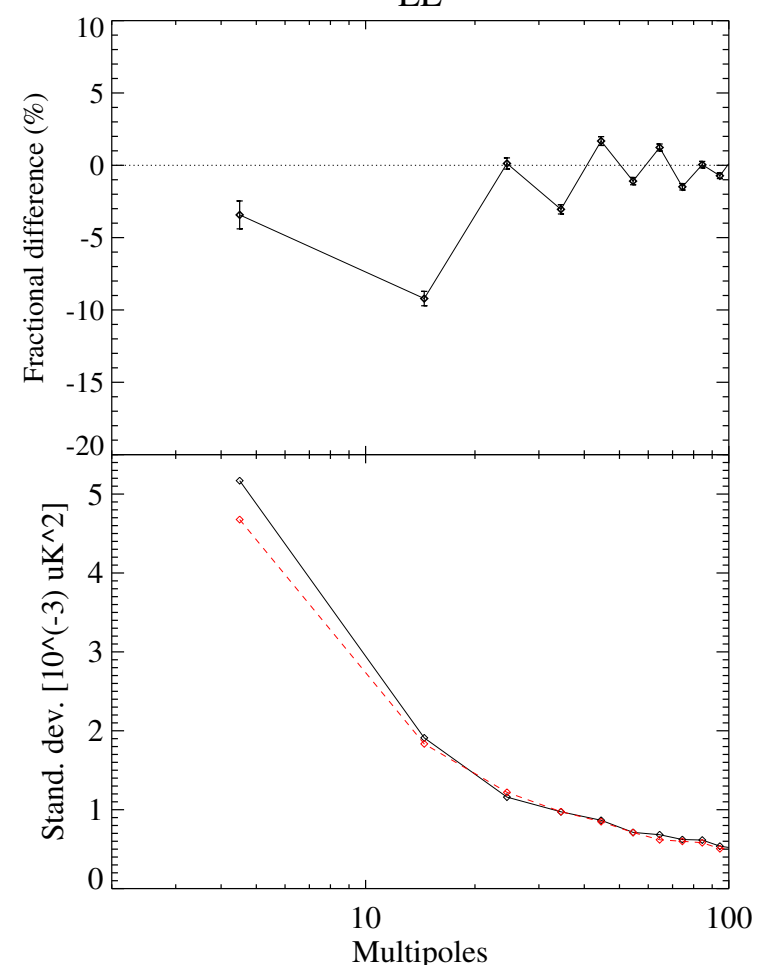

BB

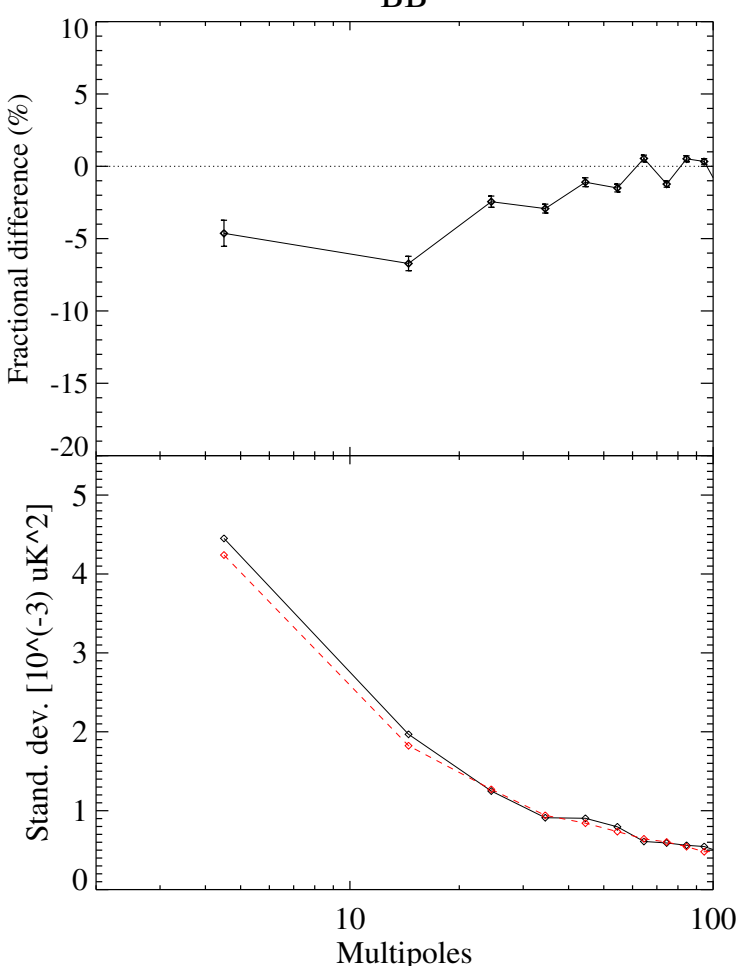

Figure 12. Comparison of $E E$ and $B B$ angular power spectra between the cases accounting for and ignoring the crosscorrelated noise in the map-making algorithm. Spectra have been estimated from 20 noise-only Monte Carlo realizations, assuming 2 detectors and one year of operation. On the top: fractional difference of $E E$ (left) and $B B$ (right) average spectra between the two cases. On the bottom: $E E$ (left) and $B B$ (right) spectrum standard deviations of the cases accounting for (dashed red line) and ignoring (solid black line) cross-correlation. Standard deviations correspond to the dispersion of the simulations.

egy. We first describe our model of the effect and the simulations performed. We then introduce a correction approach and show to which accuracy the effect could be reduced. 


\subsection{Model of the bandpass mismatch effect}

As discussed in Sect. 2 (see in particular Eq. 2.2), a detector observing the sky at the frequency $v_{0}$ with a polarizer oriented at an angle $\psi_{t}$ at time $t$ measures the quantity:

$$
d_{t}\left(v_{0}\right)=I_{p(t)}\left(v_{0}\right)+Q_{p(t)}\left(v_{0}\right) \cos \left(2 \psi_{t}\right)+U_{p(t)}\left(v_{0}\right) \sin \left(2 \psi_{t}\right)+n_{t},
$$

where $I_{p(t)}\left(v_{0}\right), Q_{p(t)}\left(v_{0}\right)$ and $U_{p(t)}\left(v_{0}\right)$ are the Stokes parameters at the position $p(t)$ on the sky measured in the local reference frame and $n_{t}$ is the random instrumental and photon noise.

For a given sky pointing, each of the Stokes parameters $(I, Q, U)$ receives contributions due to the emission of different components $c$. For simplicity, let us model the intensity in terms of these components. After integrating over the detector bandpass, the total flux par unit steradian received by a detector from the sky can be written as (see Hoang et al. (2017) for details on this model):

$$
\frac{d F}{d \Omega}=\int \sum_{c} g(v) f_{c}(v, p) d v
$$

where $g(v)$ is the detector bandpass, $f_{c}(v, p)$ the emission spectrum of the $c$ component, which can depend on the pixel. After calibrating on the $\mathrm{CMB}$, the intensity reads:

$$
I\left(v_{0}\right)=I_{\mathrm{CMB}}\left(v_{0}\right)+\sum_{c \neq \mathrm{CMB}} \gamma_{c}(p) I_{c}\left(v_{0}\right),
$$

where $I_{c}\left(v_{0}\right)$ is the mean intensity of component $c$ at a reference frequency $v_{0}$ and $\gamma_{c}(p)$ the relative amplitude of component $c$ in CMB temperature units, which is slightly pixel dependent if the component spectra depend on the sky region considered. Since the signal has been calibrated on the CMB, the factor $\gamma_{\mathrm{CMB}}$ is normalized to unity. The component amplitude coefficient, defined for the reference frequency $v_{0}$, can be related to the transmission of the band and the spectrum of the component by:

$$
\gamma_{c}=\left.\frac{\int g(v) f_{c}(v) f_{c}\left(v_{0}\right)^{-1} d v}{\left.\int g(v) \frac{d B\left(v, T_{0}\right)}{d v}\right|_{\nu_{0}} d v} \frac{d B\left(v, T_{0}\right)}{d v}\right|_{\nu_{0}},
$$

where $T_{0}$ is the mean temperature of the $\mathrm{CMB}$ and $B\left(v, T_{0}\right)$ is the blackbody spectrum of the CMB. The quantity $\gamma_{c}$ is close to 1 for a chosen $v_{0}$ near the center of the band. A similar relationship applies for the $Q$ and $U$ Stokes parameters.

The expression above describes an ideal situation and does not include real-world complications such as beam asymmetries and miscalibration. We follow this approach in order to isolate the bandpass mismatch effect. Potential couplings with other systematic effects are ignored at this stage, in the spirit of the discussion in Sect. 1.

The problem of bandpass mismatch can be understood by calculating the data model for the sky signal for a set of detectors $\{(i)\}$. Each detector $(i)$ in the set will have its own $\gamma_{c}^{(i)}$ which can be written as

$$
\gamma_{c}^{(i)}=\bar{\gamma}_{c}+\delta \gamma_{c}^{(i)}
$$

where $\bar{\gamma}_{c}$ is the mean of scaling parameter $\gamma$ for the set $\{(i)\}$ and the component $c$ and $\delta \gamma_{c}^{(i)}$ is its deviation from this mean. The data model for the sky signal can now be written, using a vector notation in boldface, as

$$
\mathbf{d}^{(i)}=\sum_{c} \bar{\gamma}_{c}\left[\mathbf{I}_{c}+\mathbf{Q}_{c} \cos (2 \psi)+\mathbf{U}_{c} \sin (2 \psi)\right]+\sum_{c \neq \mathrm{CMB}} \delta \gamma_{c}^{(i)}\left[\mathbf{I}_{c}+\mathbf{Q}_{c} \cos (2 \psi)+\mathbf{U}_{c} \sin (2 \psi)\right]+\mathbf{n} .
$$


The first term on the right hand side is the 'ideal' sky signal with all components including CMB, while the second term is the leakage term of non-CMB components due to their different bandpasses. Since the signal for each detector has been calibrated on the CMB, we expect $\delta \gamma_{C M B}$ to be zero, so it is absent from the second term.

\subsection{Simulations of the bandpass mismatch effect}

We evaluate the impact of the mismatch on a set of simulations of the data using a simplified version of the Planck sky model (PSM, see Delabrouille et al. (2013)), which comprises only two foreground components (thermal dust and synchrotron) and CMB at $145 \mathrm{GHz}$, at HEALPix resolution $N_{\text {side }}=$ 1024, and symmetric Gaussian beams of FWHM 7.6'. We use four detectors, with polarization angles evenly spaced at intervals of $45^{\circ}$, at the boresight, and with varying square bandpasses with a $1 \%$ random error on the edge of the bands. This gives similar variations of the $\gamma$ parameters than for the most pessimistic cases of Planck filters. Nevertheless, we expect less variations between future mission detector filters. The sky component is integrated over the corresponding spectrum following Eq. 6.4, using a top hat instrumental bandpass. In simulations, we have included the complexity of component spectra as modelled in the PSM, and consequently the resulting $\gamma$ parameters are pixel dependent. We have run simulations of pure signal and of signal plus white noise separately, using the nominal noise level expected for CORE, observing for one year using the baseline CORE scanning strategy as discussed in Sect. 4 above.

As described in Sect. 2, we produce intensity and polarization maps using the maximum likelihood estimator for the Stokes parameters, Eq. (2.3). In the first step we ignore the correction for bandpass leakage and use the same pixelization as the input maps to avoid introducing additional pixelization effects. Maps of the timeline noise are made separately and subtracted from total maps. This is justified since the map-making method which has been used is linear, and so instrumental noise will be purely additive.

In the absence of bandpass leakage, that is, if all $\gamma_{c}^{(i)}$, for each detector $(i)$ are identical, and there is sufficient modulation of the polarization angle $\psi$, we expect $\widehat{Q_{p}}=\bar{Q}_{p}$ and $\widehat{U_{p}}=\bar{U}_{p}$, the actual signal on the sky with no additional bias, for all pixels given our noiseless simulations.

By comparing the resulting polarization maps with the input, we estimate the impact of bandpass leakage on the polarization measurement for the chosen detector set. We compute the $\widehat{Q}$ and $\widehat{U}$ maps and compute the $E E$ and $B B$ power spectra of the difference with the input CMB $Q$ and $U$ maps after masking $25 \%$ of the sky where the Galactic dust emission is the brightest. The resulting power spectrum of the residual signal (see Fig. 13) is above the primordial $B$-mode signal even for $r=0.1$, and is thus completely unacceptable for measuring $r=0.001$. It is also above the lensing signal for $\ell<100$ (see, again, Fig 13). The prediction for any number of detectors $N_{\text {det }}$ can be obtained by rescaling the power spectrum of a factor $4 / N_{\text {det }}$ (since the result described here is for four detectors), assuming independent filter variations between detectors. The scaling of the spectrum with the inverse of the number of detectors is demonstrated in Hoang et al. (2017).

\subsection{Correction algorithm}

We now describe an approach to correct the data for bandpass mismatch that consists in estimating jointly the map $Q$ and $U$ Stokes parameters as well as the leakage component using as input data timelines built from the individual detectors at $145 \mathrm{GHz}$ bands and the templates built from $350 \mathrm{GHz}$ and $90 \mathrm{GHz}$ recovered intensity maps for thermal dust and synchrotron respectively. Correction methods specific to the Planck mission were implemented at the map as well as timeline level and are detailed in Planck Collaboration et al. (2015a) and Planck Collaboration et al. (2016g). We now develop a 
more generalized correction algorithm based on the model of the data introduced in the last section and describe the simulation employed to validate it.

The baseline focal plane design for the CORE mission uses detectors with dual polarization sensitivity in one single focal plane pixel, as well as pairs of single polarization detectors with orthogonal polarization sensitivity scanning the sky along the same trajectory. The former can directly be differenced to cancel intensity and get a polarization signal, while the latter can also be differenced but after correcting for the appropriate time-shift. Differencing in this way the timelines of two orthogonal detectors, we obtain

$$
\begin{aligned}
\mathbf{d} & =\frac{1}{2}\left(\mathbf{d}^{(a)}-\mathbf{d}^{(b)}\right) \\
& =\sum_{c} \bar{\gamma}_{c}\left[\mathbf{Q}_{c} \cos (2 \psi)+\mathbf{U}_{c} \sin (2 \psi)\right]+\frac{1}{2}\left[\sum_{c \neq \mathrm{CMB}}\left(\delta \gamma_{c}^{(a)}-\delta \gamma_{c}^{(b)}\right) \mathbf{I}_{c}\right]+\left(\mathbf{n}^{(a)}-\mathbf{n}^{(b)}\right) \\
& =\mathbf{Q} \cos (2 \psi)+\mathbf{U} \sin (2 \psi)+\sum_{c \neq \mathrm{CMB}} y_{c} \mathbf{I}_{c}+\mathbf{n},
\end{aligned}
$$

and we are left with a reduced set of Stokes parameters $[\mathbf{Q}, \mathbf{U}]$ from the CMB and Galactic sources, $\mathbf{I}_{c}$ are the timelines from reference foreground intensity maps with $y_{c}$ their corresponding amplitude, each of them given by $\frac{1}{2}\left(\delta \gamma_{c}^{(a)}-\delta \gamma_{c}^{(b)}\right)$, and $\mathbf{n}$ is the noise term.

In equation 6.7, the first term on the right hand side is the term of interest (a linear combination of the polarization Stokes parameters), while the other two are nuisance terms, a bandpass leakage term proportional to a sum of foreground components, and a noise term $\mathbf{n}$.

By recasting our data set in the form of equation 6.7, we have isolated a leakage term which is a sum of bandpass mismatch coefficients times foreground intensity templates. If the bandpass mismatch coefficients $y_{c}$ are perfectly known by calibration, our measurements can be considered as linear combinations of $\sum \mathbf{Q}_{c}$ (sky Stokes $Q$ in channel $c$ ), $\sum \mathbf{U}_{c}$ (sky Stokes $U$ in channel $c$ ), and additional foreground intensity maps $\mathbf{I}_{c}$. The system can be inverted in the usual way. If however the calibration of the bandpasses is not perfect, we want to solve also for $y_{c}$.

We propose to correct for bandpass mismatch terms with the following approximation. Assume that we have at hand measured templates for the foregrounds. Such measurements can be obtained directly for CORE intensity data, either at other frequency (i.e. higher frequency for a dust template, or lower for a synchrotron template), or at the reference frequency of the channel of interest after component separation in intensity. Such templates are not perfect, i.e.

$$
\mathbf{I}_{c}=k \widetilde{\mathbf{I}}_{c}+\delta \mathbf{I}_{c},
$$

where $k$ is a global scaling factor, and $\delta \mathbf{I}_{c}$ the difference between the scaled template and the real foreground map. By replacing the (unknown) $\mathbf{I}_{c}$ by its expression in terms of the (known) template $\widehat{\mathbf{I}}_{c}$ in Eq. 6.7, neglecting the second-order term proportional to $y_{c} \delta \mathbf{I}_{c}$, and absorbing the global scaling factor $k$ in $y_{c}$, we get, in matrix-vector notation

$$
\mathbf{d}=\mathbf{A m}+\mathbf{T y}+\mathbf{n},
$$

where $\mathrm{A}$ is a reduced pointing matrix with has two non-zero elements in each row

$$
\mathbf{A}_{t p(t)}=\left[\cos \left(2 \psi_{t}\right) \sin \left(2 \psi_{t}\right)\right],
$$

$\mathbf{m}$ is the reduced set of Stokes parameters, containing only $[\mathbf{Q}, \mathbf{U}]$ from both the CMB and the Galactic sources, $\mathbf{T}$ is built from the known foreground template maps $\widetilde{\mathbf{I}}_{c}, \mathbf{y}$ is the set of amplitudes of the leakage of the corresponding templates in that difference timeline, and $\mathbf{n}$ is the noise term. 
We find an unbiased estimator $\widetilde{\mathbf{m}}$ free (to first order) from the leakage term with a standard generalized least square estimator of the form

$$
\begin{aligned}
& \widetilde{\mathbf{m}}=\left(\mathbf{A}^{T} \mathbf{N}^{-1} \mathbf{F}_{\mathbf{T}} \mathbf{A}\right)^{-1} \mathbf{A} \mathbf{N}^{-1} \mathbf{F}_{\mathbf{T}} \mathbf{d}, \\
& \widetilde{\mathbf{y}}=\left(\mathbf{T}^{T} \mathbf{N}^{-1} \mathbf{F}_{\mathbf{A}} \mathbf{T}\right)^{-1} \mathbf{T} \mathbf{N}^{-1} \mathbf{F}_{\mathbf{A}} \mathbf{d},
\end{aligned}
$$

and

$$
\begin{aligned}
& \mathbf{F}_{\mathbf{A}}=\left\{\mathbf{1}-\mathbf{A}\left(\mathbf{A}^{T} \mathbf{N}^{-1} \mathbf{A}\right)^{-1} \mathbf{A}^{T} \mathbf{N}^{-1}\right\}, \\
& \mathbf{F}_{\mathbf{T}}=\left\{\mathbf{1}-\mathbf{T}\left(\mathbf{T}^{T} \mathbf{N}^{-1} \mathbf{T}\right)^{-1} \mathbf{T}^{T} \mathbf{N}^{-1}\right\} .
\end{aligned}
$$

We identify the terms $\mathbf{F}_{\mathbf{A}}$ and $\mathbf{F}_{\mathbf{T}}$ as operators that filter out the component of the signal in the respective space of $\mathbf{A}$ and $\mathbf{T}$. Also, the noise covariance term $\mathbf{N}$ for the case of white noise is diagonal given by $\sigma^{2} \mathbf{1}$, where $\sigma$ is the standard deviation of the white noise, and it cancels out in the previous equations. We thus freely drop the noise covariance term $\mathbf{N}$. It is to be noted that this algorithm can be suited for any number of systematic effects whose leakage signal can be modelled as a nuisance term proportional to a temporal template as shown in equation (6.7). An analogous approach to subtract temporal templates was implemented in Poletti, D. et al. (2017) for the Polarbear experiment using the direct estimation of maps as in equation (6.11).

To make matters computationally feasible we perform our correction iteratively by first estimating the amplitudes $\mathbf{y}$ of the templates using Eq. (6.12), then use these to perform a simple GLS map-making by subtracting the leakage term from the timeline by

$$
\widetilde{\mathbf{m}}=\left(\mathbf{A}^{T} \mathbf{A}\right)^{-1} \mathbf{A}^{T}(\mathbf{d}-\mathbf{T} \widetilde{\mathbf{y}})
$$

We test here this correction algorithm on the simulations described in the previous subsection. For our templates we simulate intensity maps for thermal dust at at $350 \mathrm{GHz}$ and synchrotron at $90 \mathrm{GHz}$ using the PSM. The $E E$ and $B B$ power spectra of the leakage maps are shown in Fig. 13. The algorithm reduces the leakage by more than two orders of magnitude in power. The residual after correction for a set of 4 detectors is now comparable to the primordial B-modes for a level of $r$ in the 0.001-0.01 range, and below the lensing signal for $\ell \geq 10$.

As already emphasized, the power spectrum of the leakage after averaging $N$ pairs of detectors will be reduced by $N$. The CORE $145 \mathrm{GHz}$ channel comprises 144 detectors (72 pairs). Hence we estimate that the residual leakage after correction and averaging all detectors of the channel will be one order of magnitude or more below the target sensitivity at all angular scales. If necessary, this approach can be extended to second order to further reduce the residuals.

\section{Asymmetric beam}

The convolution of the CMB signal with an asymmetric beam will cause leakage between intensity and and $E$ - and $B$-mode polarization when $I, Q$ and $U$ maps are reconstructed using the generalized least squares solution of equation 2.3 without proper measures to take into account this beam asymmetry. Given that the primordial intensity signal is much larger than the polarization signal, the most important effect is the temperature-to-polarization leakage.

In this section, we both investigate the amplitude of this effect for representative CORE beams, and propose methods to correct this effect by data processing. To this effect, we first generate simulations of the CORE beams at $145 \mathrm{GHz}$ for the cross-Dragonian design of the telescope. Beam maps 


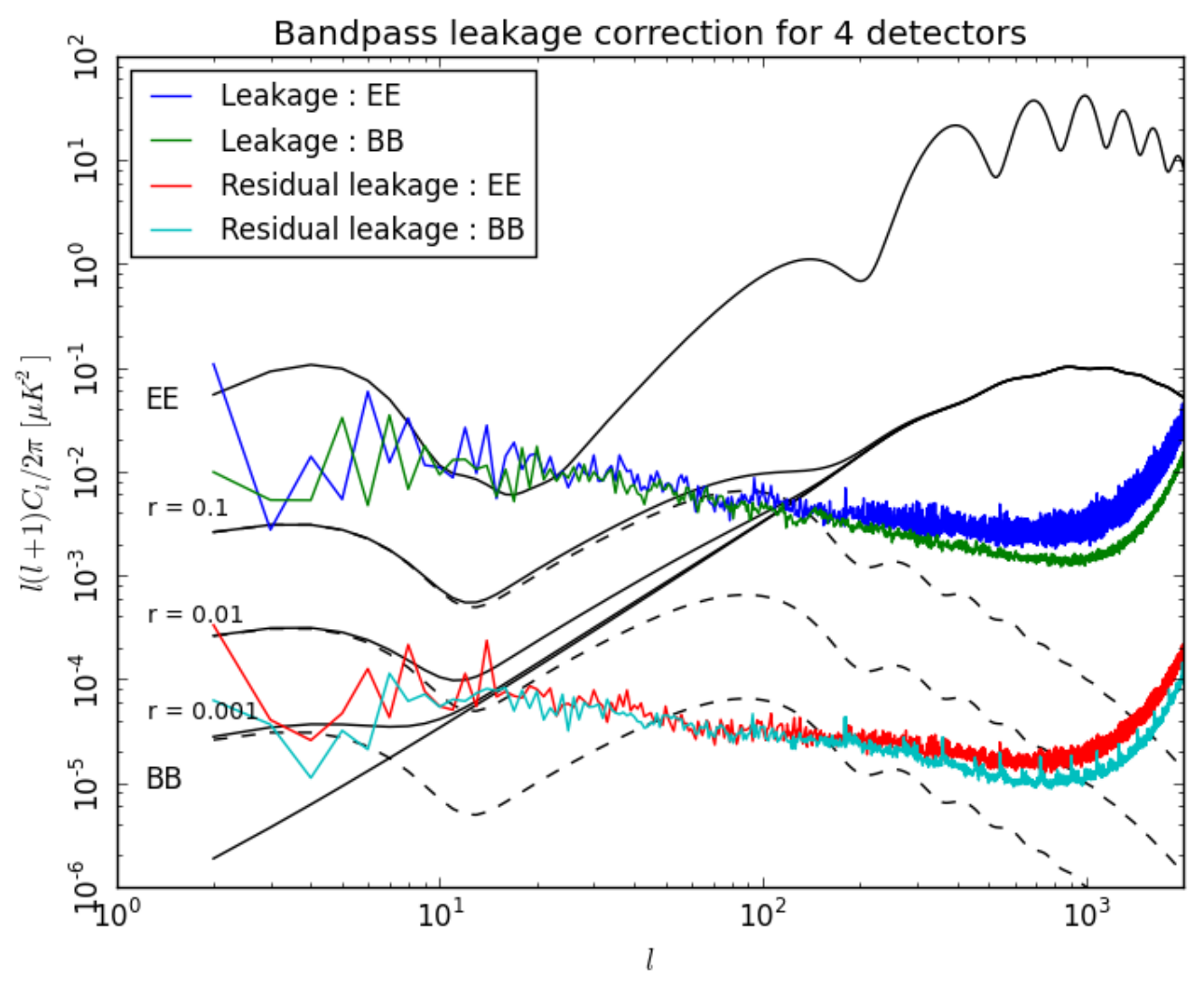

Figure 13. $E E$ and $B B$ power spectra of bandpass mismatch leakage for a set of 4 detectors at $145 \mathrm{GHz}$ before and after implementing the correction algorithm described in the text. After subtracting the leakage induced by thermal dust and synchrotron to first order, the power is reduced by more than two orders of magnitude (from blue to red for $E E$ and from green to light blue for $B B$ ).

are computed using GRASP for three locations in the focal plane, the boresight, a 'high' detector 4 degrees from the boresight towards the spin axis, and a 'low' detector 4 degrees from the boresight in the opposite direction (note the locations of the high and low detectors considered here differ from those assumed in Sect. 4). In each location all components of the beams (Stokes parameters $I, Q$, $U$ and $V$ ) are simulated for two orthogonal polarizations, $x$ and $y$, where $x$ is aligned with the scan direction. This arrangement of detectors does not reflect the layout of a real focal plane, which would naturally have detectors of the same frequency grouped together. Instead it is designed to explore the variation of the beam shapes with position in the focal plane using a representative CMB-dominated frequency channel as a test case. Figure 14 shows the Stokes parameters of the $x$-polarized beams for the three locations. The corresponding $y$-polarized beams are almost identical in shape, and only differ in the sign of the $Q$ and $U$ Stokes parameters.

These beams are then used to simulate the CMB signal observed by the CORE instrument. Two complementary paths have been followed in this investigation. The first starts with simulated CORE timelines using a high-resolution implementation of direct-space convolution. Maps of $I, Q$, and $U$ are reconstructed using equation 2.3, and beam asymmetry effects are corrected using a reobservation of the reconstructed map of $I$. In the second, we directly use a power-spectrum approach, the QuickPol formalism (Hivon et al. 2017), to estimate and correct for biases in the power spectra. 

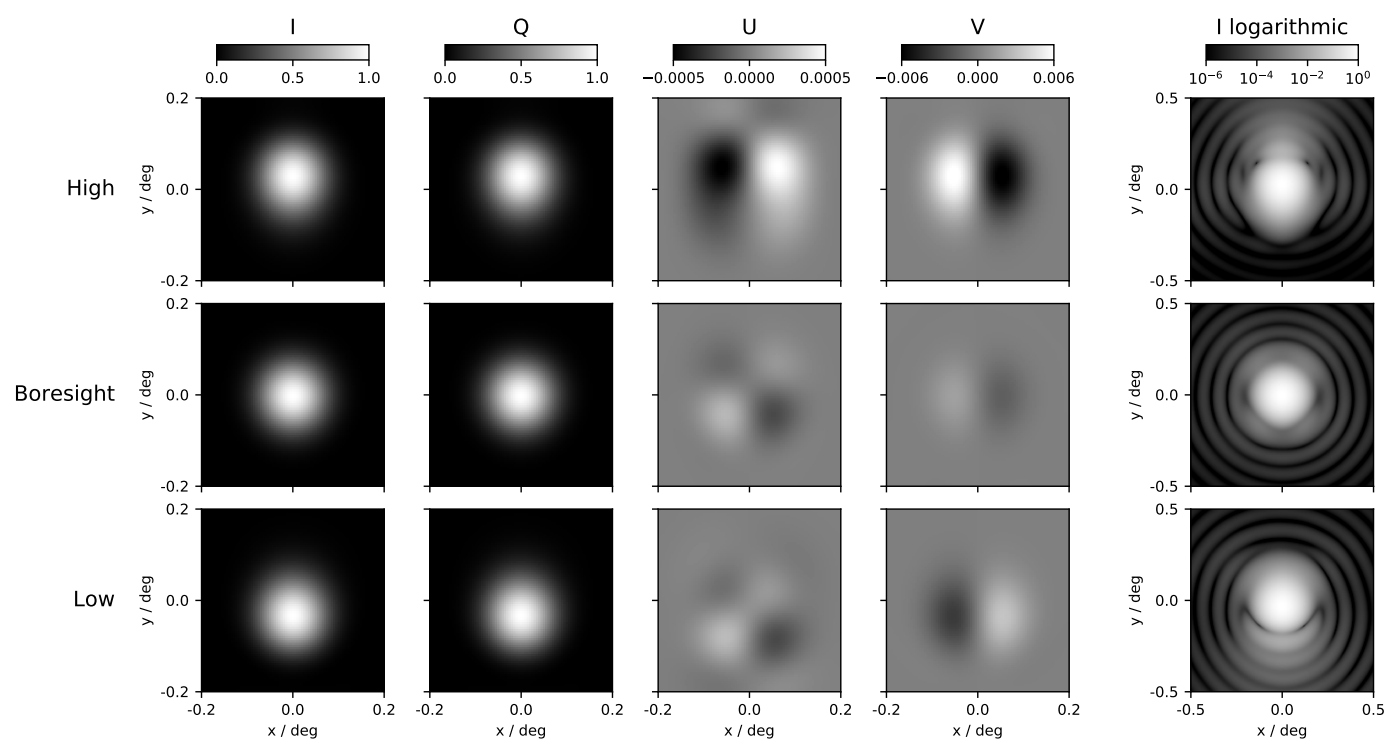

Figure 14. Simulated $145 \mathrm{GHz}$ beams for the cross-Dragonian design of the CORE telecope. The three rows show the beams for the high, boresight, and low detectors (see text for details). The first 4 columns show the $I, Q, U$, and $V$ Stokes parameters of the beams on a linear scale. The last column shows a wider view of the $I$ Stokes parameter on a logarithmic scale. The Stokes parameters have been normalized to unity at the peak in intensity.

We now describe simulations and results for these two approaches.

\subsection{Real space convolution and first-order de-projection}

The beams of the proposed CORE mission are relatively small. The impact of beam asymmetry must be calculated with sufficient numerical precision. To this effect, we implement a real-space convolution of the CMB sky by a pixelized beam map, which allows us to simulate a realistic timeline signal for any beam shape. Deviations of the beam from a symmetric Gaussian shape project onto the time ordered signal in a manner that reflects the orientation of the beam along the scan.

The real space beam convolution technique uses a method specifically designed for a spinning experiment, which breaks up a $N \times N$ pixelized beam map into $N$ rows that are aligned with the scanning direction. In each row, every beam pixel observes the same signal, up to a time shift and a global scaling term, that is, the contribution of the row to the total signal is obtained by onedimensional convolution of a signal timeline by the corresponding beam cut (a row of beam pixels). The data timeline is cut in smaller periods with some overlap to perform the convolution in practice. After each timeline is convolved in Fourier space by the corresponding beam row, the sum of all the individual rows gives us the full convolved timeline. This method scales as $N \times \log (N)$ instead of $N^{2}$ as would be the case if the contribution of all $N^{2}$ pixels was computed independently for each data point.

The real space convolution has the added advantage of reducing sub-pixel effects, for a more accurate timeline signal that avoids part of the sky pixelization effects. This is illustrated in Fig. 15, which compares the timeline obtained by real-space convolution with an $8^{\prime}$ Gaussian symmetric beam, with that obtained by scanning with a pencil beam the map after convolution with the same beam in harmonic space. The real-space convolution, which uses beam pixels of size $\simeq 1^{\prime}$, produces a smoother signal, without the numerical artifacts due to the discontinuities between the pixels of 
the HEALPix map. All simulations performed here use high resolution maps at $N_{\text {side }}=4096$ for adequate sampling of the underlying CMB signal, and hence good numerical precision.

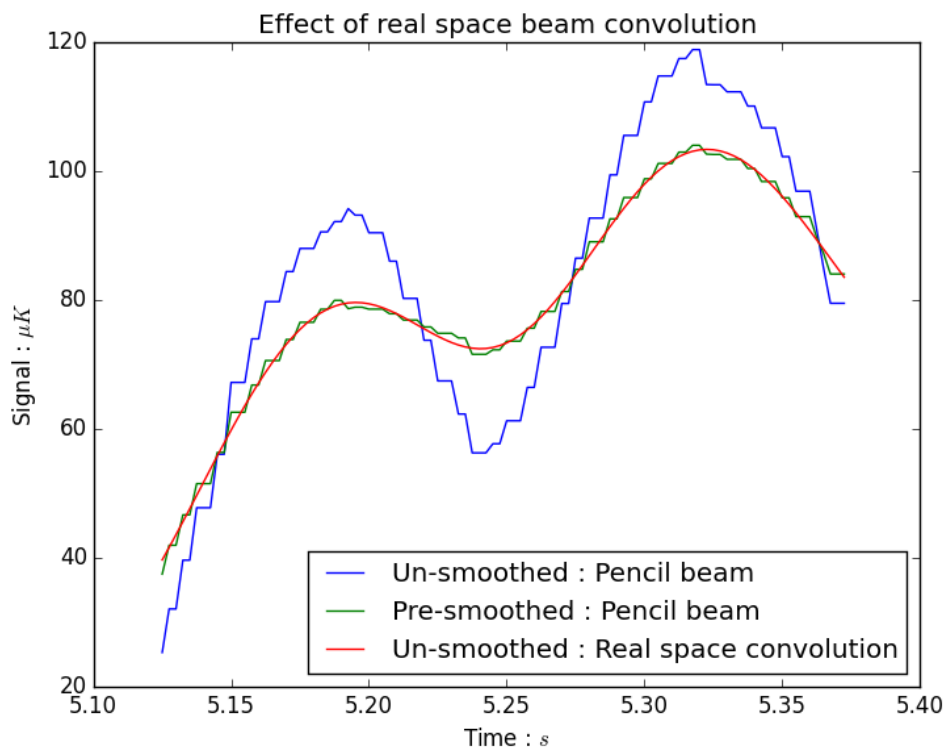

Figure 15. Comparison of signals obtained from various beam convolution methods. The signal obtained using a pencil beam to scan a map pre-smoothed in harmonic space with an $8^{\prime}$ beam (green) is compared to the signal obtained using the real-space convolution technique to scan an un-smoothed map with the same pixel size (red). All calculations are performed using HEALPix maps at $N_{\text {side }}=4096$, corresponding to a pixel size of about $52^{\prime \prime}$. The green and the red signals signals follow each other closely, although we distinguish small discontinuities in the former due to pixelization, which are absent in the latter.

Simulations for CORE are performed by scanning an unsmoothed CMB map using the pixelized beam maps of Fig. 14 with the real space convolution described above. Maps made from the resulting timelines suffer from the temperature-to-polarization leakage due to the asymmetric beams and from sub-dominant cross-polarization terms, projected onto the reconstructed 'observed' map as a function of the scanning strategy. Reconstructed maps of $Q$ and $U$ (and hence $E$ and $B$ ), are thus contaminated with leakage from temperature to polarization. These effects can be quite large, as illustrated in Fig. 16, in particular for detectors near the edge of the focal plane, which have more asymmetric beams than those of detector located at the boresight, for which even the $E$ modes would be significantly contaminated. We note however that the temperature-to-polarization leakage is reduced for maps obtained with two detectors instead of one, when these two detectors are in the same location, but are sensitive to orthogonal polarizations. This happens because the $I$ beams of two such detectors are very similar, which leads to much of the leakage being cancelled in the map-making stage.

To correct for the temperature-to-polarization leakage in the map due to the beams, we consider an intensity map that has been observed by our given set of detectors as a template. This template, however, is not perfect because it contains noise and errors due to the beam asymmetry itself. Still, we can use this template to estimate the intensity-to-polarization leakage to first order. To do this, the template is deconvolved in harmonic space using an effective average symmetric beam that best fits the angular resolution of the channel. In practice, this deconvolution is performed only up to a certain limit of $\ell$ (see Banerji and Delabrouille (2017) for details). This deconvolved map is then 
re-scanned using the same scanning strategy and the intensity beam of the detector of interest. The resulting timeline will therefore contain the intensity signal, along with higher order leakage terms due to the asymmetric beams, and noise, as well as the initial leakage terms being observed again. When projected onto $(Q, U)$ maps, this timeline propagates an estimate of the intensity leakage terms to the polarization map for that particular detector (or detector set when several timelines are combined). Thus to first-order, we can use it to estimate the temperature-to-polarization leakage due to asymmetric beams. The leakage in the observed polarization maps is then cleaned by subtracting the estimated leakage maps. At first order, this method does not correct for cross-polar leakage that mixes $E$ and $B$ modes, which would require additional modelling terms (see discussion about systematics in Delabrouille et al. (2017) for a general introduction).

After such first-order correction, as seen in Fig. 17, the leakage for a single high/low detector is significantly reduced, below the $B B$ lensing signal for $l \leq 300$. For one boresight detector the residual leakage is at the level of the primordial BB spectra for $r=0.001$ for the first few 10's of multipoles. For the pair of boresight detectors, the residual leakage is dominated by cross-polar leakage (which is not corrected for in this analysis) and is well below the primordial $B B$ spectrum $\mathrm{f}$ for all $l \leq 200$, even for $r=0.001$.

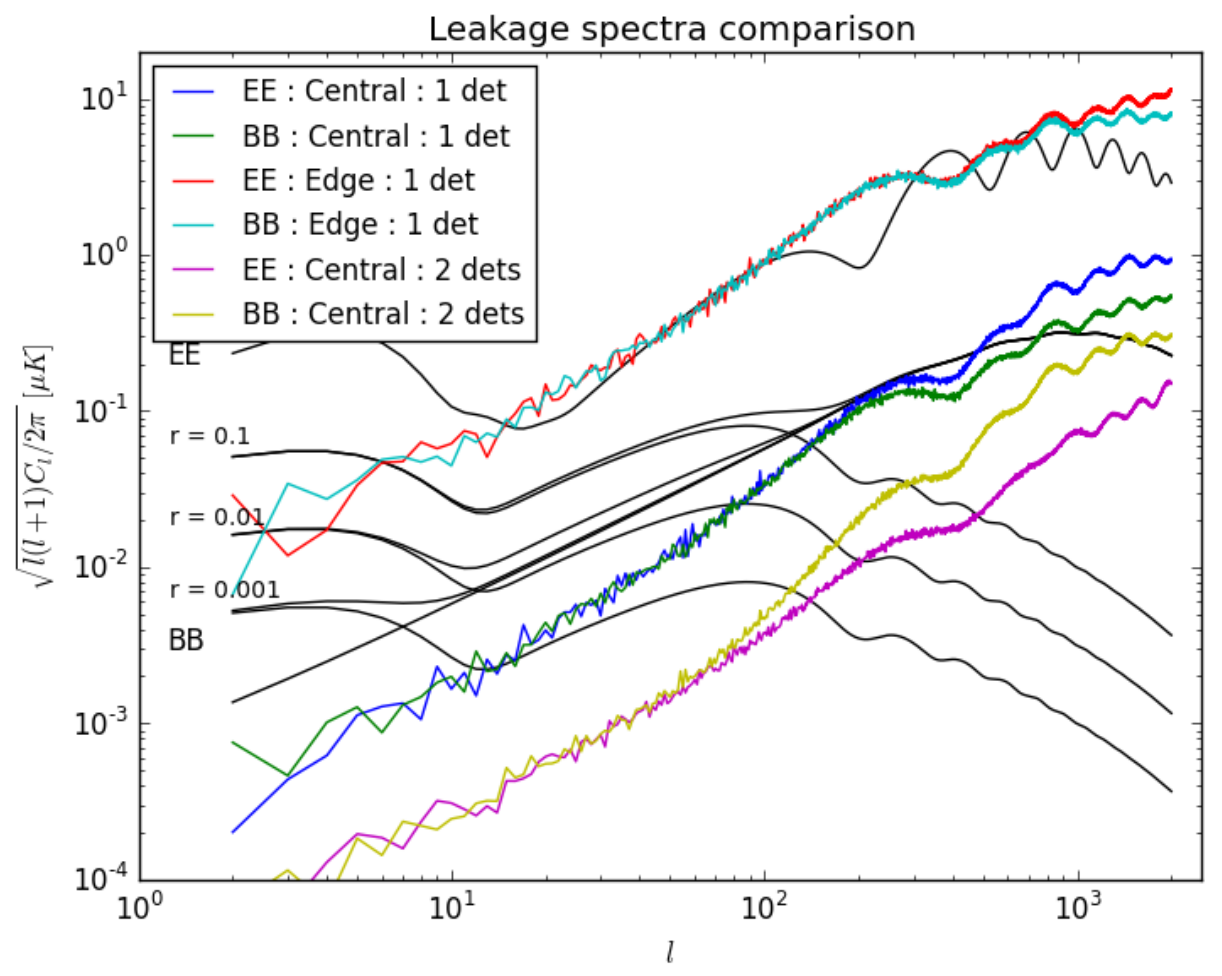

Figure 16. $E E$ and $B B$ spectra of leakage due to beam asymmetry and cross-polarization. The spectra are computed from the difference of the output CMB map with the input map. The effect of the additional asymmetry of the edge detectors is reflected in the increase in the leakage spectra as compared to the boresight detector. The leakage is decreased when using a pair of orthogonally polarized detectors due to better modulation of angles. 


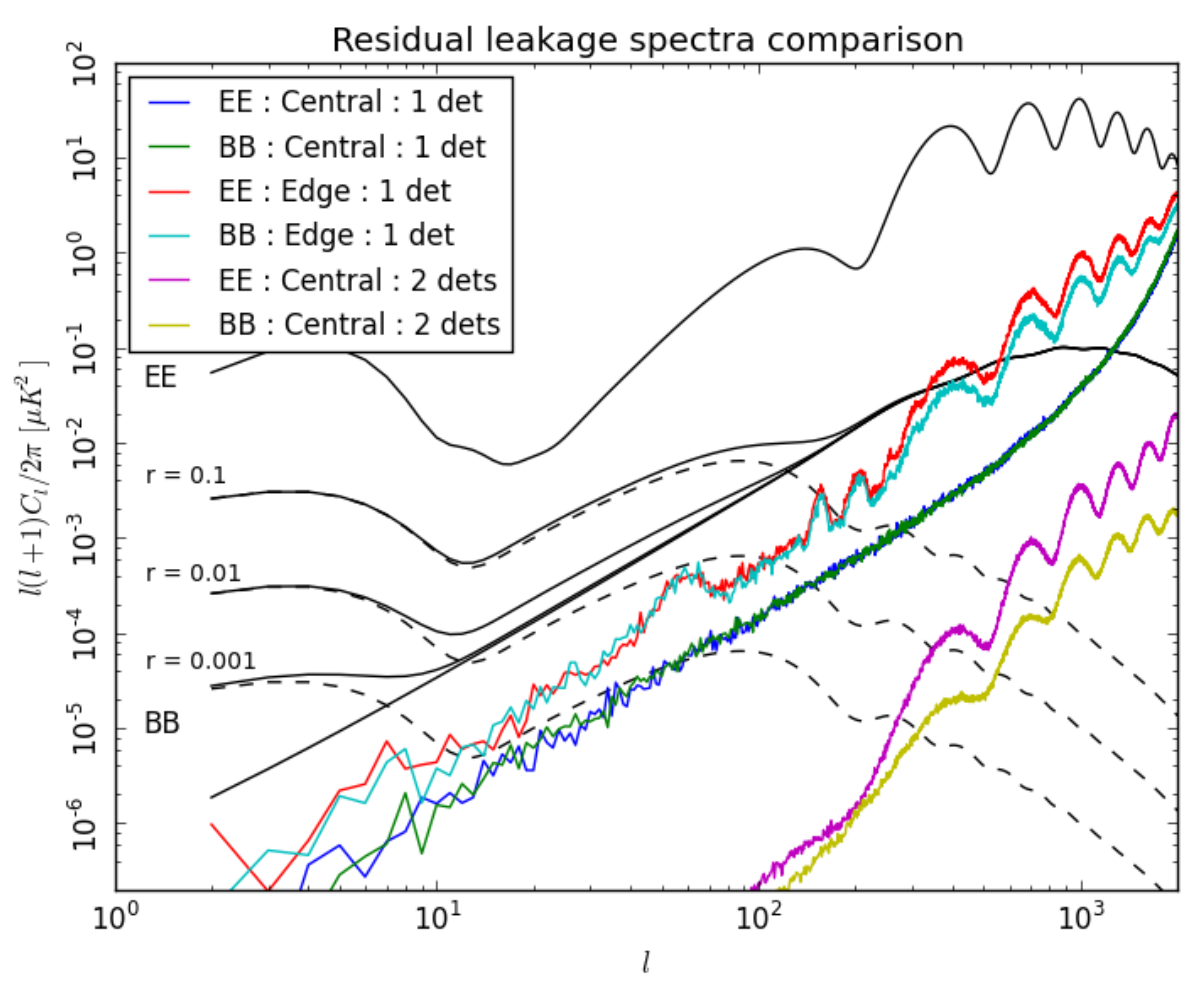

Figure 17. $E E$ and $B B$ spectra of the residual beam leakage after correction by the real-space method. The residual spectra are dominated by cross-polar leakage and by pixelization noise due to the process of deconvolution and re-observation of the template map.

\subsection{Harmonic space}

An alternative way to account and correct for the effect of the beam leakage is to work at the angular power spectrum level. The QuickPol formalism (Hivon et al. 2017), allows such a description, as illustrated in Fig. 18 where its predictions are compared to simulations of observations of either a single detector or the pair of detectors at the boresight. The agreement with the simulations is very good, including at very large multipoles, where the sub-pixel effects, discussed in Sec. 9, dominate.

The formalism only needs a description of the detector scanning strategy, in the form of the statistics of the orientation of the detectors on the sky in a subset of sky pixels, the spherical harmonic representation of the beams, and some extra description of the detectors: orientation in the focal plane, gain calibration, polarization efficiency. It returns a beam matrix describing how, at each multipole $\ell$, the input $T T, T E, E E$, and $B B C_{\ell}$ power spectra are related to the measured $\widetilde{C}_{\ell}$ spectra, that is

$$
\widetilde{C}_{\ell}^{X Y}=\sum_{X^{\prime} Y^{\prime}} W_{\ell}^{X Y, X^{\prime} Y^{\prime}} C_{\ell}^{X^{\prime} Y^{\prime}}
$$

with $X, Y, X^{\prime}, Y^{\prime} \in\{T, E, B\}$. Such a $W_{\ell}^{X Y, X^{\prime} Y^{\prime}}$ beam matrix can be used in the cosmological analysis of the measured power spectra, in order to determine the model that best fits the data. Since the algorithm is very fast, it can be used for a Monte-Carlo determination of the systematics-related error bars on the final $C_{\ell}$. It simply has to be run multiple times while varying the values of the instrumental parameters known with limited accuracy, such as the polarization efficiency or even the beam shape. 

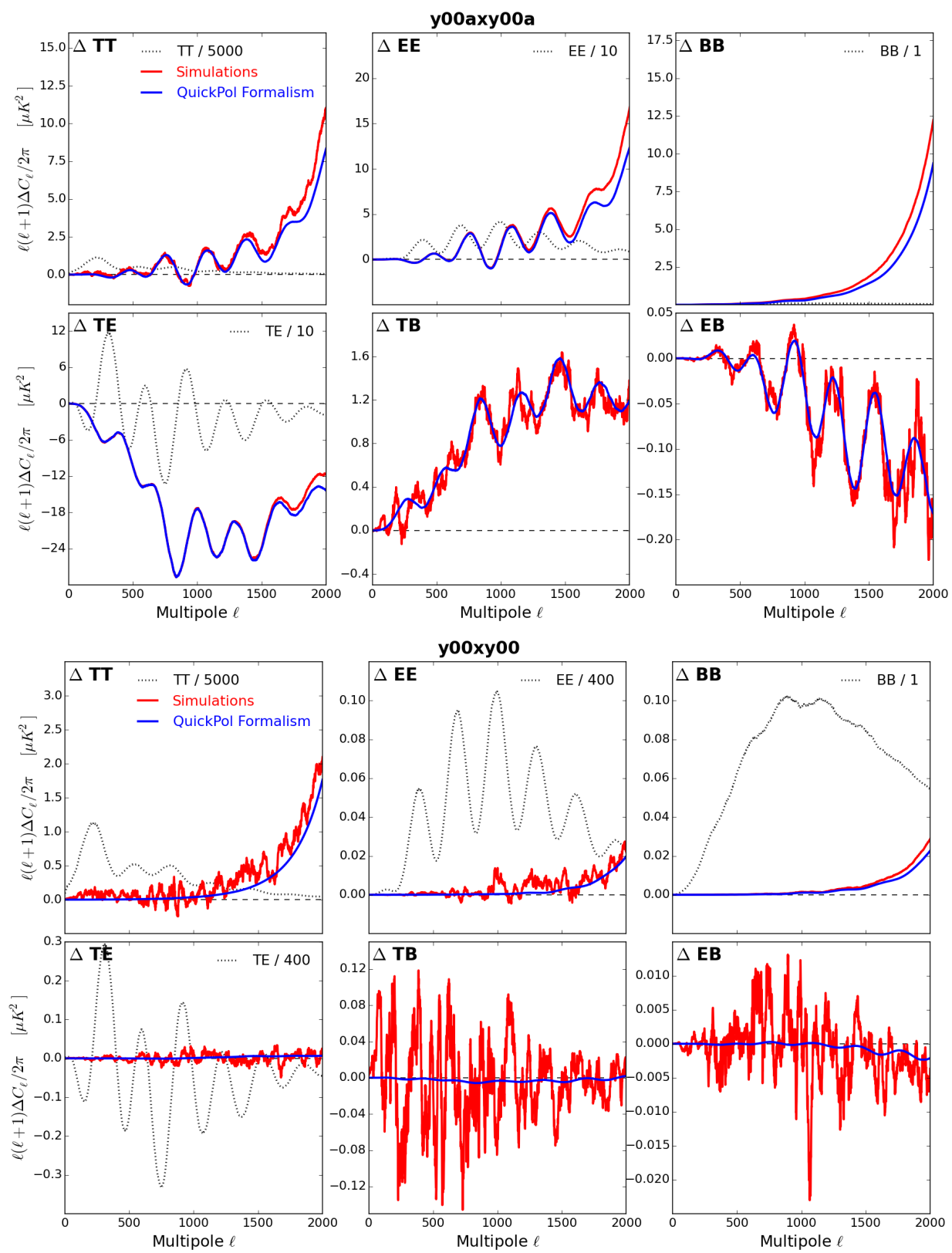

Figure 18. Discrepancy between the measured $\ell(\ell+1) C_{\ell}$ and the input, smoothed with $\Delta \ell=31$, for a single CORE detector (6 upper panels) and for a pair of orthogonally polarized detectors in the same location (6 lower panels) at the boresight. Results obtained in noiseless simulations of CORE observations (red lines) are to be compared with those of the QuickPol formalism (blue lines). In panels where it does not vanish, a fraction of the input power spectrum is also shown in black dots for comparison.

\subsection{Beam asymmetry conclusions}

In the absence of fast modulation of polarization such as with a rotating half-wave plate, the nonidealities of the beams could hinder the precise measurements of primordial $B B$ spectrum by CORE. However, as we have shown, we have at our disposal two different and complementary approaches to deal with such systematics, one map based, and the other one power spectrum based. They both 
allow us to simulate rapidly and precisely the impact of CORE beam non-idealities on the maps or the power spectra, and can therefore be used to respectively clean the maps of most of these systematic effects, and account precisely for these systematics in the cosmological analysis of the measured power spectra, even with an imperfect knowledge of the instrument features.

While these investigations must be pursued in more detail, no technical limitation has been identified from beam asymmetry effects for the CORE space mission. Further investigations must also address the simultaneous deprojection of all potential systematics, and are postponed to further study.

\section{Calibration}

In this section we discuss the systematic effects that can potentially affect the quality of the photometric calibration of CORE's detectors. By 'photometric calibration', we mean the process of converting the output produced by a detector into the amplitude of the signal entering CORE's optical system. Assuming that the output of the detectors depends linearly on the input signal entering the optical system, the result of a photometric calibration is a timeline of multiplicative calibration factors that convert the output of a detector into thermodynamic temperature. In the case of Planck HFI, the stability of the detectors was good enough to allow the use of a constant calibration factor over the 2.5 years of the nominal mission (Planck Collaboration et al. 2014b, 2015a). In this work, we aim to determine how well we can detect changes in the calibration factors of CORE detectors that unfold over timescales of the order of a few hours to a few days.

The ability to track changes in the value of the calibration factors is affected by the strength of the signal used for the calibration. As was the case with WMAP and Planck, we plan to use the dipolar signal induced by the motion of the Sun with respect to the CMB rest frame as a calibration signal. We call this signal the 'dipole' for brevity. For simplicity, in this analysis we neglect the contribution of the time-varying orbital dipole, caused by the yearly motion of the CORE spacecraft with respect to the Solar System. This component, called the orbital dipole, is usually $\sim 10 \%$ of the solar dipole, the component due to the motion of the Sun with respect to the CMB rest frame (Planck Collaboration et al. 2015b). Including this component would have improved the estimation of the calibration factors at the expense of a more complex analysis pipeline.

We have developed an improved version of the calibration code used for Planck LFI (Planck Collaboration et al. 2015b), because code has the ability to track changes of the calibration with time $^{6}$. Using an approach similar to the one described by Tristram et al. (2011), the LFI calibration algorithm, named DaCapo, is an hybrid calibration and map-making tool which is able to estimate the calibration factors, the $1 / f$ noise timelines, and the sky map simultaneously, by means of a modified destriping algorithm. We have rewritten the code from scratch in Python 3 and have incorporated it in TOAST. DaCapo uses a maximum-likelihood approach which is similar to destriping techniques employed to solve map-making problems, and it shares with them a number of concepts (See Sect. 2 above). We have modified the original algorithm in order to allow the timescale on which the calibration is assumed to be constant to vary. In the original version for the Planck LFI, this timescale was assumed to be one pointing period of $40-60 \mathrm{~min}$.

We present a brief overview of the algorithm here; for additional details, the reader can refer to the source code of the programs we have used in this analysis (Tomasi 2016).

\footnotetext{
${ }^{6}$ Unlike HFI, which used stable bolometric detectors based on transition edge sensors (TES), LFI implemented squarelaw detectors based on high electron mobility transistors (HEMT), which have shown non-negligible changes (of the order of several percent) in the gain constants over the 4 years of the extended mission.
} 
The DaCapo algorithm models the output of a detector scanning the sky using the following approximation:

$$
s\left(t_{i}\right)=G_{k}\left(T_{i}+D_{i}\right)+b_{n}+n_{i},
$$

where $s\left(t_{i}\right)$ is the signal measured by the detector at time $t_{i}, T_{i}$ is the temperature of the sky (including Galactic signals, CMB, point sources, etc.) ${ }^{7}, D_{i}$ is the amplitude of the solar and orbital components of the Doppler dipole (due to the motion of the spacecraft on its orbit in the solar system, and of the solar system with respect to the CMB: as previously mentioned, we neglect the orbital contribution in the following analysis), $n_{i}$ is a white-noise term, $G_{k}$ is the gain, and $b_{n}$ is an offset which keeps track of slow fluctuations due to $1 / f$ noise. The DaCapo algorithm obtains a maximum-likelihood estimate of $G_{k}, T_{i}$, and $b_{n}$ given $s$ and $D$. The last quantity is given by:

$$
D(\mathbf{x}, t)=T_{\mathrm{CMB}}\left(\frac{1}{\gamma(1-\beta \cdot \mathbf{x})}-1\right),
$$

where $T_{\mathrm{CMB}}$ is the mean temperature of the CMB, $\beta=\mathbf{v} / c$ is the velocity of the spacecraft with respect to the CMB rest frame, $\gamma=(1-\beta)^{1 / 2}$, and $\mathbf{x}$ is the direction of the detector's main beam axis at time $t$. The values for $T_{\mathrm{CMB}}$ and $\mathbf{v}$ have been taken from Mather et al. (1999) and Planck Collaboration et al. (2016a), respectively.

The quantities $G$ and $b$ in Eq. (8.1) are indexed by $k$ and $n$ instead of $i$. This relates to the fact that the sampling rate of these two quantities must be smaller than the sampling rate of $s$ : in this way, the number of unknowns of the calibration problem is smaller than the number of variables. We call the inverse of the sampling rate of $G_{k}$ the 'calibration period'; its fluctuations unfold on periods of the order of several hours; the inverse of the sampling rate of $b_{n}$ is the 'offset period', and it must be shorter than the knee frequency of the $1 / f$ noise component. Unlike the code used in Planck Collaboration et al. (2015b), we allow the calibration period to be different from the offset period; in this way, we have an additional free parameter that can be tuned to optimize the quality of the calibration. Specifically, this change allows us to make the calibration period longer than the offset period, thus potentially improving the detection of the dipole signal thanks to a better $\mathrm{S} / \mathrm{N}$ without degrading too much the estimation of the $1 / f$ component. This change is important in the context of the CORE proposal, as we expect CORE detectors to be more stable than those of Planck LFI.

We solve for the unknowns in Eq. (8.1) by minimizing the quantity

$$
\chi^{2}=\sum_{i} \frac{\left(s_{i}-s\left(t_{i}\right)\right)^{2}}{\sigma_{i}^{2}},
$$

where $s_{i}$ is the sample acquired by the detector, and $s\left(t_{i}\right)$ is the model in Eq. (8.1). To make the problem well-posed, Planck Collaboration et al. (2015b) adds the constraint that the map $\mathbf{m}$ of the sky temperature $T$ be orthogonal to the dipole map $D$, and that the monopole of map $T$ be zero. Using Lagrange multipliers, the coupling between the minimization of Eq. (8.3) and these additional constraints leads to the following solution ${ }^{8}$ :

$$
\mathbf{F}^{T} \mathbf{N}_{w}^{-1} \mathbf{Z F a}=\mathbf{F}^{T} \mathbf{N}_{w}^{-1} \mathbf{Z s},
$$

\footnotetext{
${ }^{7}$ We do not attempt to model polarized signals in Eq. (8.1), as we have found that the systematic effect induced by this approximation is far smaller than other systematics discussed later.

${ }^{8}$ In the following equations, we adopt the convention of using bold to indicate vectors and matrices, and italic to indicate scalar values. The $i j$ coefficient of matrix $\mathbf{m}$ is therefore indicated as $m_{i j}$.
} 

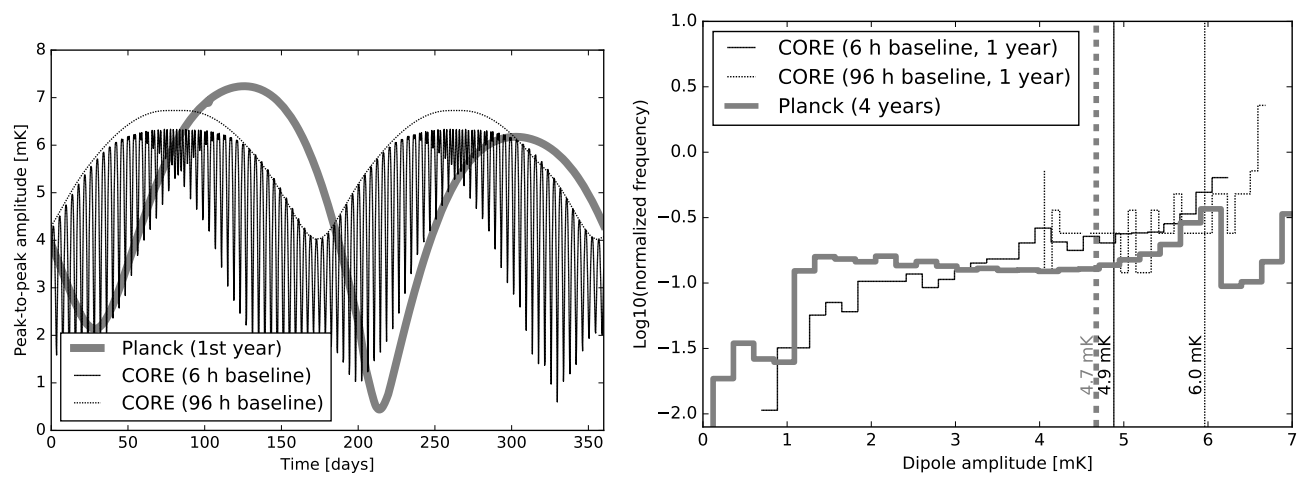

Figure 19. Left: Peak-to-peak amplitude of the hourly dipole signal as seen by CORE (thin black line for 6 hour baseline and thin dotted line for 96 hour baseline) and Planck (thick grey line). CORE's faster variations will allow to pinpoint the calibration constant more accurately than was possible with Planck data. Right: Distribution of the amplitudes shown in the left plot. Although the maximum amplitude of the hourly dipole signal seen by CORE is smaller than those seen by Planck, the tail at low amplitudes is considerably smaller. Thus, the median amplitude seen during each hour of CORE's sky observation is $5.8 \mathrm{mK}$, instead of Planck's value of $4.7 \mathrm{mK}(\sim 20 \%$ larger $)$.

where $\mathbf{N}_{w}$ is the covariance matrix of the white noise component $n_{i}$ in Eq. (8.1), a is a vector containing all the values of $b_{n}$ and $G_{k}, \mathbf{F}$ is defined by the following relation

$$
\sum_{j} F_{i j} a_{j} \equiv G_{k}\left(D_{i}+\sum_{p} P_{i p} m_{p}\right)+b_{n}
$$

and

$$
\begin{aligned}
\mathbf{Z} & =\mathbf{I}-\tilde{\mathbf{P}}\left(\mathbf{M}+\mathbf{N}_{w}^{-1}\right)^{-1} \tilde{\mathbf{P}}^{T} \mathbf{N}_{w}^{-1}, \\
\mathbf{M} & =\tilde{\mathbf{P}}^{T} \mathbf{N}_{w}^{-1} \tilde{\mathbf{P}} \\
\left(\mathbf{M}+\mathbf{N}_{w}^{-1}\right) & =\mathbf{M}^{-1}-\mathbf{M}^{-1} \mathbf{m}_{c}\left(\mathbf{m}_{c}^{T} \mathbf{M}^{-1} \mathbf{m}_{c}\right)^{-1} \mathbf{m}_{c}^{-1} \mathbf{M}^{-1},
\end{aligned}
$$

where $\mathbf{I}$ is the identity matrix, $\mathbf{m}_{c}$ is a two-column matrix containing the map of the dipole signal $D$ and a constant map with each pixel set to 1 , and $\tilde{\mathbf{P}}$ is the pointing matrix $\mathbf{P}$ where each non-zero element has been multiplied by the corresponding gain $G_{k}$.

In the next paragraphs, we discuss how this model can be applied to timelines of signals acquired by detectors scanning the sky using CORE's scanning strategy, the kind of statistical and systematics errors we should expect using this approach, and the estimated quality of the results.

\subsection{Time dependence of the dipole signal}

The quality of the estimates for $G_{k}$ (Eq. 8.1) depends on the peak-to-peak variation in the amplitude of the dipole $D$ during each calibration period. We have therefore studied the variation in time of the dipole signal $D$ as observed by a typical CORE detector.

Figure 19 shows the expected peak-to-peak variation of $D$ within one calibration period, for different values of this period. We have considered periods going from 6 hours to 4 days; the latter case is interesting, since this is the precession period used in CORE's baseline scanning strategy. Therefore, using calibration periods of this length produces large coverages within each period. We compare our estimates with the peak-to-peak variation of $D$ as seen by a Planck detector LFI27M-00), 

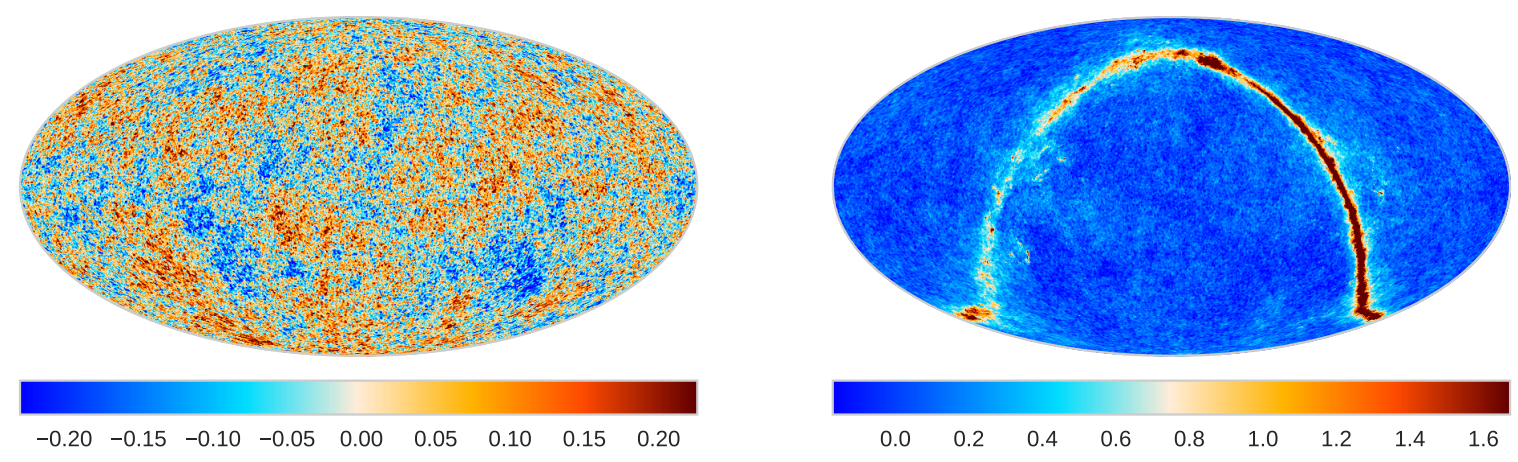

Figure 20. Foreground maps used to estimate the quality of the calibration of CORE detectors. Temperatures are expressed in $\mathrm{mK}$. Left: The CMB map produced by Planckin the Ecliptic coordinates. Right: The Planck HFI $143 \mathrm{GHz}$ temperature map in the Ecliptic coordinates.

whose calibration periods lasted 1 hour: CORE's scanning strategy will lead to larger peak-to-peak variations in the calibration signal $D$, thus potentially improving the quality of the calibration.

In the next sections we show the results of a number of simulations run under different assumptions, and quantify the quality of CORE's calibration more precisely.

\subsection{Systematics}

We run a number of simulations to estimate the impact of different systematics on the calibration of the CORE detectors. Our simulations are generated using the following approach:

1. Created timelines by scanning sky maps under the assumption of CORE's proposed scanning strategy ${ }^{9}$. The signal in the timelines is

$$
s\left(t_{i}\right)=G \times\left(I_{i}+Q_{i} \cos 2 \psi+U_{i} \sin 2 \psi\right)+\tilde{n}_{i},
$$

where $G$ is the gain, constant throughout the whole simulation, $I_{i}, Q_{i}$, and $U_{i}$ are the Stokes parameters of the pixel being observed at time $t_{i}$, and $\tilde{n}_{i}$ is a $1 / f$ plus white noise realization. The knee frequency of the $1 / f$ noise has been set to $20 \mathrm{mHz}$. We produce two sets of timelines using two sky maps: the first one being the Planck 2015 CMB map, the second one being Planck $2015143 \mathrm{GHz}$ map.

2. We run DaCapo on the simulated timelines using different values of the calibration period, and compare the estimates of the gains $G_{k}$ with the input gain $G$ used in Eq. (8.9).

We consider 5 values for the calibration period $\Delta t_{G}$ : 6 hours, 12 hours, 1 day, 2 days, and 4 days. The value actually used in the analysis of the data acquired by CORE will depend on the overall stability of the detectors; the experience acquired with HFI shows that bolometers are extremely stable, and intrinsic variations are usually smaller than gain uncertainties (Planck Collaboration et al. 2015a).

The results of our simulations are shown in Fig. 21. The amplitude of the fluctuations is reduced for large values of the calibration period, as expected. For the best case $\left(\Delta t_{G}=4\right.$ days), the estimated gain is $G=34.996_{-0.008}^{+0.010} \mathrm{~K} / \mathrm{V}$ (95\% C.L.), in agreement with the input value $G=35 \mathrm{~K} / \mathrm{V}$ within $0.01 \%$.

\footnotetext{
${ }^{9}$ The code used to produce the timelines is based on TOAST. It is freely available at https://github.com/ ziotom78/create_timelines.
} 

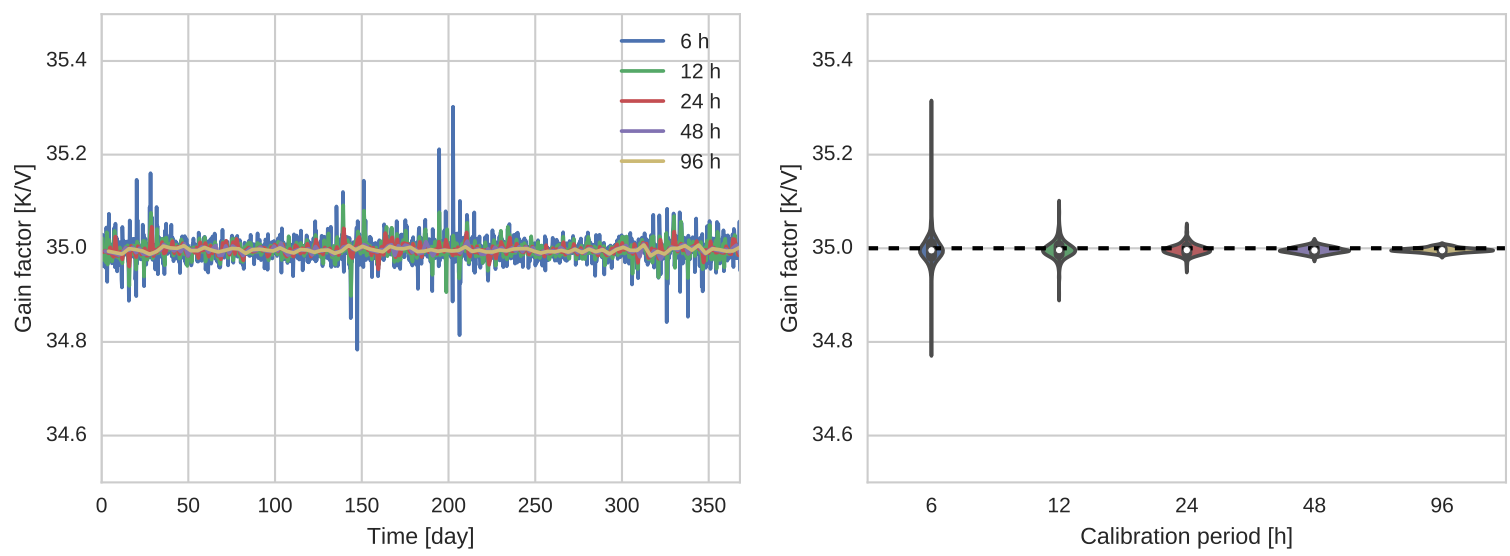

Figure 21. Left: Variation of the gains estimated in the case of a CMB-only sky. The gain $G$ used in Eq. (8.9) to produce the simulated timelines was $35.0 \mathrm{~K} / \mathrm{V}$. Right: Violin plots of the gain estimates shown on the left. Increasing the length of a calibration period reduces the dispersion of the gain estimates.

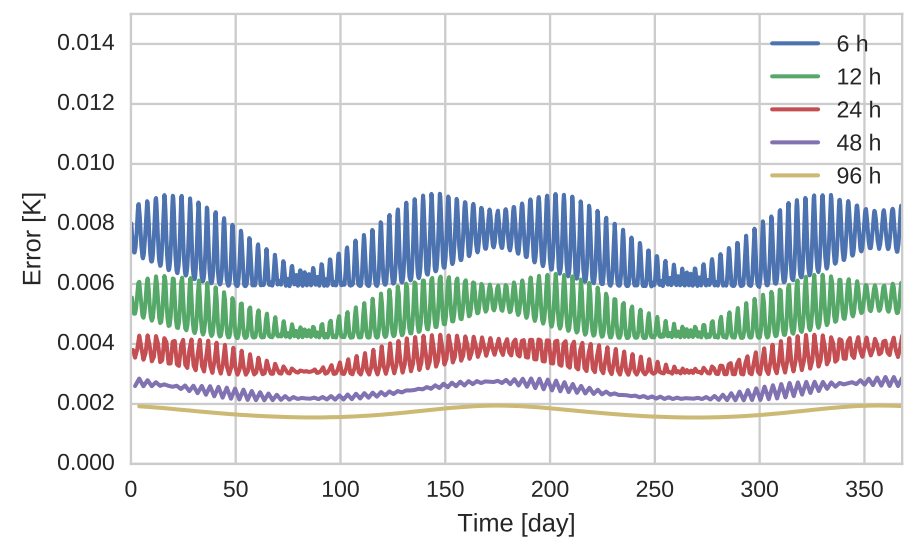

Figure 22. The uncorrelated statistical errors for the gains shown in the plot on the left of Fig. 21 in the case of a CMB-only sky for different lengths of the calibration period. The errors have been computed assuming diagonality for the gain covariance matrix, and they are therefore a lower bound. Both the short- and longperiod fluctuations in the error are anticorrelated with the amplitude of the dipole signal; see the plot on the left of Fig. 19.

The algorithm is able to estimate the error of each gain estimate, under the following assumptions:

1. Only the statistical noise is considered (that is, due to the presence of the $n_{i}$ term in Eq. (8.1));

2. No correlation is assumed between the gains of the same timeline.

This estimate is a lower bound on the true statistical error, since the latter will also include the effect of imperfect $1 / f$ cleaning and the correlation between pairs of gains. The behaviour of the errors is shown in Fig. 22: their time dependence is clearly anticorrelated with the amplitude of the dipole variations shown in Fig. 19. 


\subsection{Systematics due to the Galaxy}

The presence of a Galactic signal in the temperature measured by CORE detectors is more problematic than the presence of the CMB signal considered in Sect. 8.2, because the Galaxy shows large scale features that can be easily mistaken for a component of the dipole signal, thus causing leakage from $T$ to $D$ in Eq. (8.1). There are two effectsthat are caused by this leakage ${ }^{10}$; they depend on the relative orientation of the Galactic dipole axis with respect to the CMB dipole axis:

1. The axis of the total dipole is tilted with respect to the CMB dipole axis; this induces a timedependent bias whose exact shape depends on the scanning strategy, but in any case it is likely to produce large-scale stripes in the sky maps;

2. The amplitude of the dipole is either increased or decreased, depending on whether the angular separation between the two axes is smaller or larger than $90^{\circ}$, respectively; this effect leads to a constant offset of the gain estimates $G_{k}$, as it does not depend on the scanning strategy. The effect of this systematic effect is to change the amplitude of the CMB signal.

Both effects are potentially dangerous and should be properly characterized. One possible approach to reducing the impact of Galactic contamination would be to use foreground map templates of the form $\epsilon_{k} \mathbf{F}_{k}$ in Eq. (8.1), where $k$ indexes different foregrounds (synchrotron, CO lines, dust, etc.), and solve for the unknown scalar factors $\epsilon_{k}$; this is the approach used by Planck to characterize bandpass mismatches among HFI bolometers (Planck Collaboration et al. 2015a). A possibly better approach would be to do the calibration in tandem with a component separation algorithm, using the following iterative procedure:

1. Estimate the gains $G_{k}$ ignoring the presence of the Galaxy, and produce maps of the sky signal at different frequencies;

2. Run a component-separation algorithm on the maps and estimate the Galactic signal;

3. Scan the map of the Galactic signal into a timeline, and use the gains estimated in step 1 to decalibrate this timeline;

4. Clean the data timeline using the one estimated in step 3 and repeat the process from the beginning.

For the purpose of this work, we adopt the simpler approach of applying a mask which removes the brightest parts of the Galaxy from the computation, as was done in Planck Collaboration et al. (2015b). To produce the timelines, we simulate the observation using the $143 \mathrm{GHz}$ map from the Planck 2015 data release. The masks have been created using the $353 \mathrm{GHz}$ map from the same data release to remove those sky regions where the dust signal is strongest. In Fig. 23 we show the three sky masks we use and the angular separation between the Galactic dipole axis and the CMB dipole axis as a function of the sky mask. Since the axis of the Galactic dipole in the Planck sky maps is nearly perpendicular to the $\mathrm{CMB}$ dipole axis ${ }^{11}$, slightly varying the masked region can make the separation between the two axes smaller or greater than $90^{\circ}$, thus changing the sign of the overall bias induced by the Galaxy. As the right-hand side of Fig. 23 shows, in the case of the $143 \mathrm{GHz}$ map, we expect the bias to be positive for the $90 \%$ mask, and negative in the other cases.

\footnotetext{
${ }^{10}$ Obviously, the sum of two dipolar signal on the sky is still a dipolar signal, as it can be readily proven by adding their harmonic coefficients.

${ }^{11}$ This is true for the sky maps produced by WMAP and Planck LFI and HFI, as the angle between the two dipole axes always differs from $90^{\circ}$ by only a few arcseconds.
} 

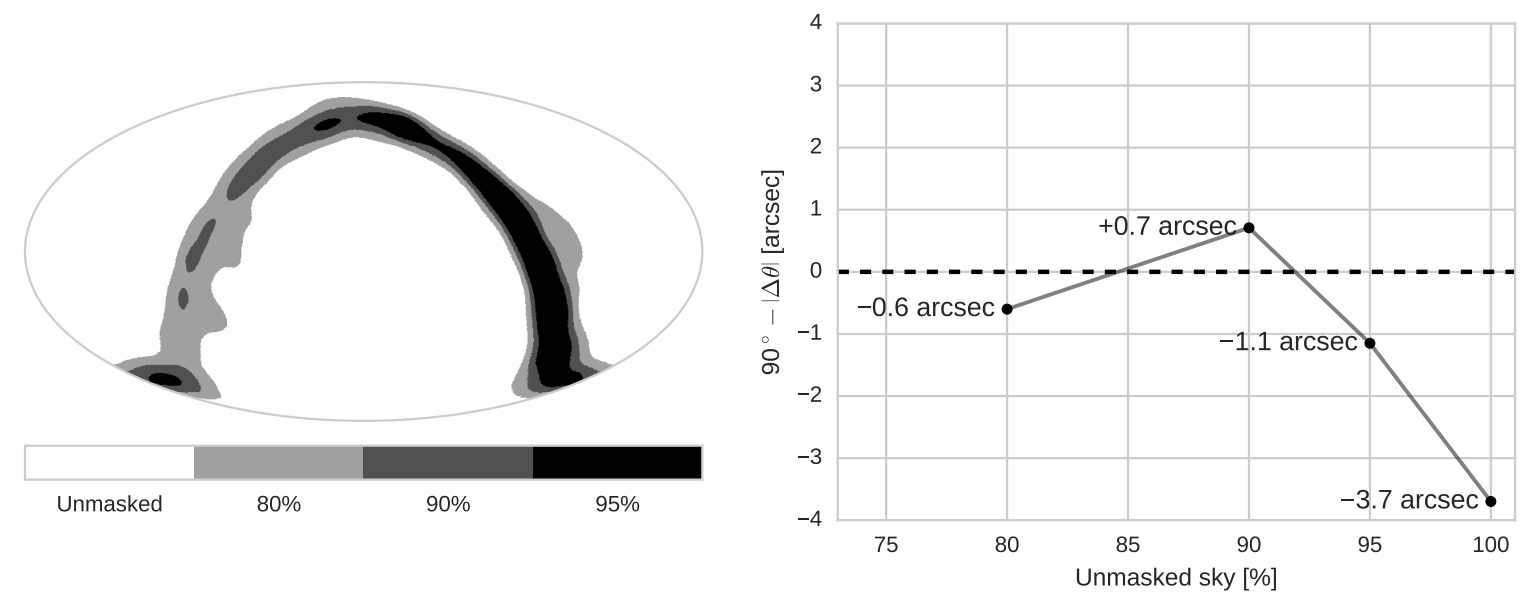

Figure 23. Left: Mollweide projection of the three masks used to remove the contribution of the Galactic dust from the data used in the calibration simulations. The masks have been created using the Planck $353 \mathrm{GHz}$ temperature maps. They are shown here in Ecliptic coordinates. Right: Angular separation between the axis of the Galactic dipole in the Planck HFI $143 \mathrm{GHz}$ temperature map and the axis of the CMB solar dipole, as a function of the Galactic mask. For every mask, the two axes are nearly perpendicular; however, in the case of the $90 \%$ mask the separation is slightly less than $90^{\circ}$. This has implications for the calibration, as shown in Fig. 24.
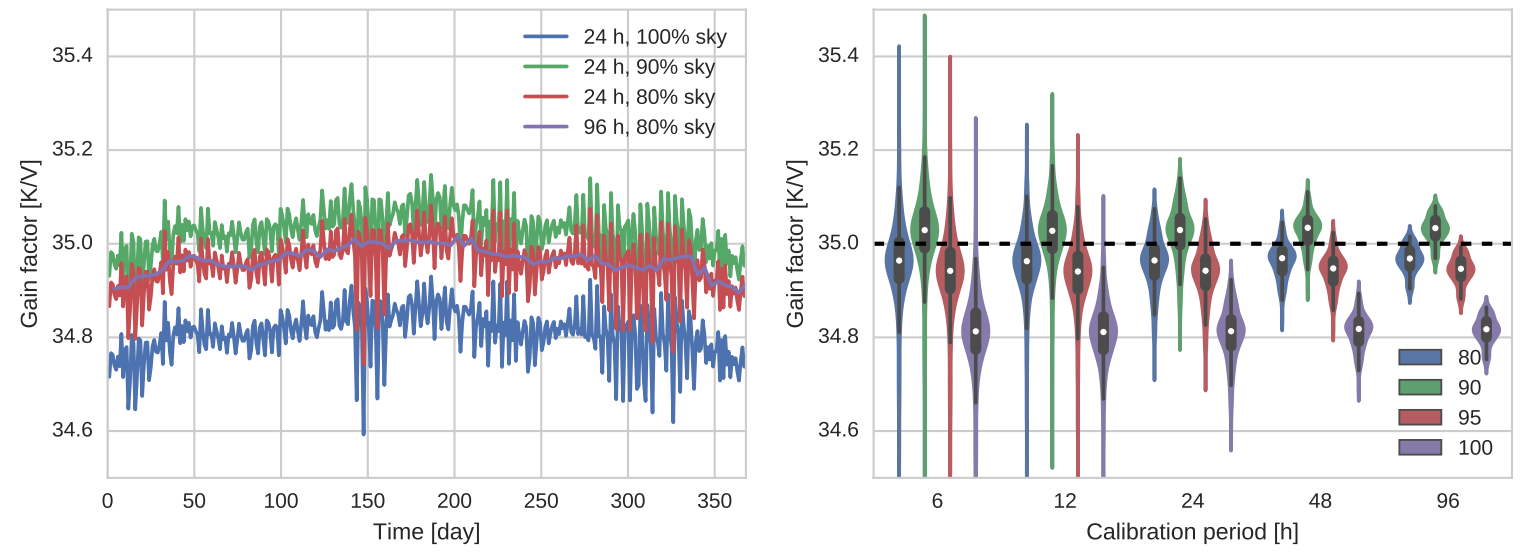

Figure 24. Variation of the estimated gains with time in the case of a realistic sky (Planck HFI $143 \mathrm{GHz}$ map). As in Fig. 21, $G=35.0 \mathrm{~K} / \mathrm{V}$. The presence of the Galaxy induces a systematic offset, mainly due to the additional dipolar component which biases the calibration. Applying masks helps in reducing this effect, as the cases with a $80 \%$ mask show. The sign of the bias is always negative except in the case of the $90 \%$ mask: this depends on the relative orientation of the Galactic dipole axis with respect to the CMB solar dipole, as explained in the text. (See also Fig. 23.)

Figure 24 shows the results of the simulations. Unlike Fig. 21, the two systematic effects we expected are now clearly evident: (1) a systematic bias in the overall level of the gains, which is positive when the $90 \%$ mask is used and negative otherwise, and (2) time-dependent fluctuations which are not reduced if longer calibration periods are used. If a calibration period of 4 days is used, and $80 \%$ of the sky is used, then the gain estimate is $G=34.97_{-0.06}^{+0.04} \mathrm{~K} / \mathrm{V}$, which should again be compared to the reference value $G=35 \mathrm{~K} / \mathrm{V}$ used in Eq. (8.9). The results are still consistent with 

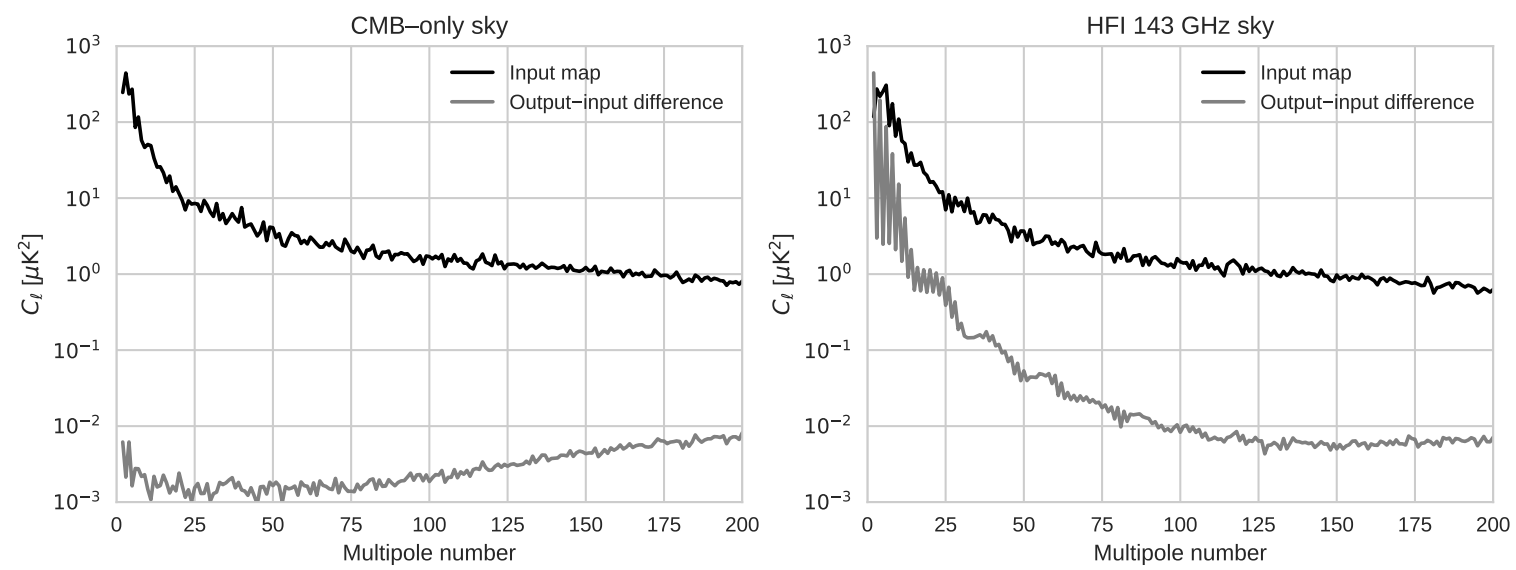

Figure 25. Left: Comparison of the power spectrum of the CMB map with the spectrum of the difference between the same CMB map and the output map produced by DaCapo. Right: The same comparison for the HFI $143 \mathrm{GHz}$ map.

the reference value, with a $0.1 \%$ error.

Our results show that residual Galactic emission outside the mask produces a systematic effect in the determination of $G$. We have tested this and found that this effect is larger than the systematic error due to neglecting polarization in the map (see Eq. 8.1), as the latter is at least one order of magnitude smaller. To assess the impact of this kind of systematic error on the scientific outcome of CORE, a more detailed set of simulations need to be carried out. However, we expect that the impact of such errors on polarization measurements will be smaller than $0.1 \%$, as the error on $G$ is highly correlated between detectors. Therefore, it should cancel when differencing data from detector pairs like the one used in our simulations (that is, two detectors oriented at $-22.5^{\circ}$ and $67.5^{\circ}$ with respect to the scan direction of motion). In fact, for a given detector pair the systematic contamination due to the Galaxy will cancel out to first order when differencing the data. When averaging $N$ such pairs, oriented the same way, residual noise will be dominated by uncorrelated contributions, thus scaling down as $1 / \sqrt{N}$.

Figure 25 shows a comparison between the power spectrum of the input map and the that of the residual map between the input and the output map produced by DaCapo. In the case of a CMB sky, the residuals are more than two orders of magnitude smaller than the map itself. In the case of a realistic sky with Galactic dust emission, the residuals become of the same order of magnitude as the signal itself at large angular scales $(\ell<10)$. To address these discrepancies, we expect to use the calibration pipeline in tandem with high quality models of the sky and component-separation methods. As a result, the overall calibration accuracy should fall between the optimistic case presented in Sect. $8.2(0.01 \%)$ and the pessimistic case discussed in Sect. $8.3(0.1 \%)$.

\section{Pointing accuracy and reconstruction uncertainty}

The variation of the temperature signal at scales smaller than the beam size may induce spurious polarized signals in two different, but closely related, ways. (1) Sub-pixel effects due to the variation of the sky signal within a map pixel can generate artefacts, that depend mainly on the distance between the nominal pixel centre and the average position of the samples in the pixel, multiplied by the local signal gradient. If the pointing were perfectly known, and once a first estimate of the signal is available, they could be corrected iteratively at the map or at the power spectrum level (Hivon et al. 


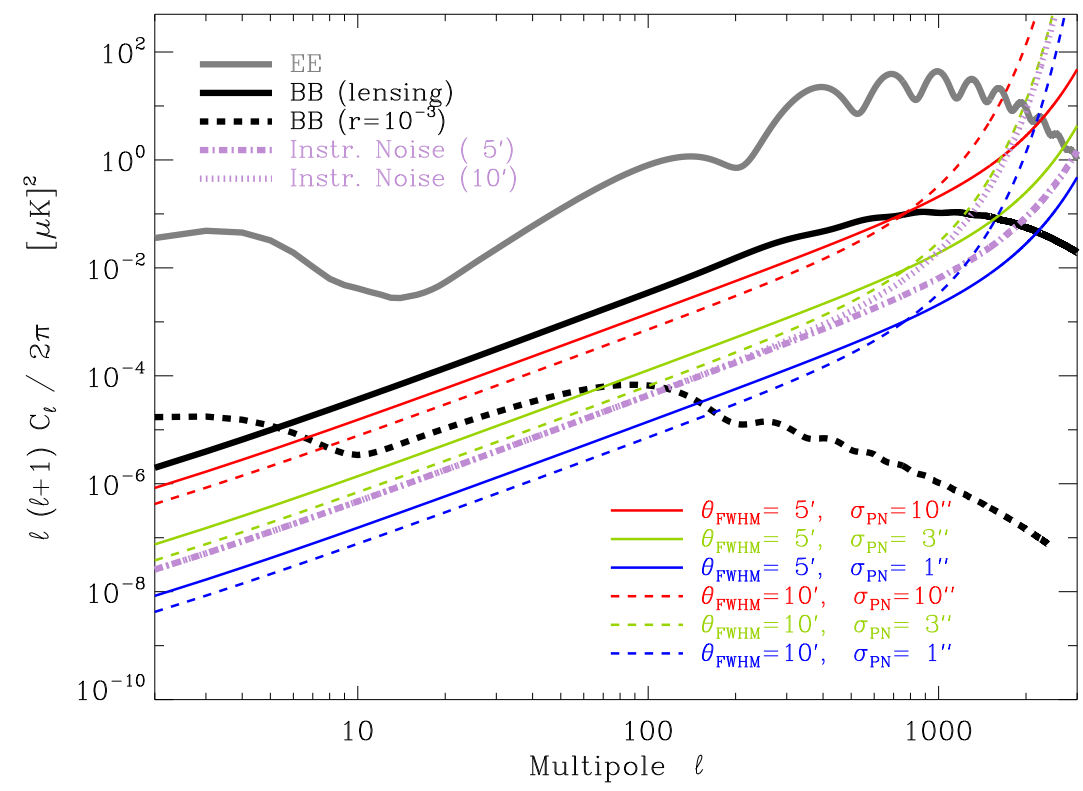

Figure 26. Angular power spectrum of the noise induced by pointing inaccuracy (red, green and blue curves), after correction of the beam window functions, compared to the $E E$ and $B B$ (either pure lensing or primordial with $r=10^{-3}$ ) spectra (grey and black curves). The instrumental noise (purple curves) is assumed to be $2 \mu \mathrm{K} \cdot$ arcmin, the expected CORE sensitivity to CMB polarization.

2017). This is, however, limited by (2) the accuracy of the pointing reconstruction (for example, a few arcsec in the case of Planck), which will create a pointing noise contaminating all power spectra at a comparable amplitude. If the pointing error in a pixel, integrated over all the samples and detectors contributing to the map, is assumed to be weakly correlated with the adjacent pixels, then the resulting pointing induced noise will also be white, with an amplitude determined by the pointing error variance and the variance of the temperature gradient. Figure 26 shows how this pointing-induced noise compares to the angular power spectra of the polarized signal and instrumental noise, for residual pointing error per pixel of 1,3, and $10 \operatorname{arcsec} r m s$ and beam FWHM of 5 and 10 arcmin. This shows that a pointing error of a few arcsec or less is enough to allow a good measurement of the $B B$ power spectrum. Furthermore, assuming that the pointing error variance is measured, correction schemes can be implemented, as proven by several CMB experiments, including Planck (Planck Collaboration et al. 2016c) and Spider.

If the pointing error is correlated between pixels, and presents some long term drifts or correlations with external factors, then specific tools would have to be developed to treat it.

\section{Conclusions}

In the context of the proposed CORE satellite mission to map CMB polarization, we have discussed the impact of the main systematic effects we expect to affect the observations, with emphasis on their projected impact on frequency maps. We have chosen to deal with one effect at a time, for the sake of carefully assessing its impact in isolation from the others. In a real world application, however, systematic effects will interact one another, implying that their combined effect cannot always be deduce by linear superposition. Their treatment will thus require a global processing pipeline. An 
accurate assessment by means of complete end-to-end simulations is needed, but will have to wait until we will possess detailed knowledge of the individual effects. We therefore defer this analysis to future work.

We have employed the TOAST simulation pipeline to generate timelines of realistic instrumental noise and beam-convolved sky signals. We have also employed the flexible generalized destriping code MADAM to produce maps with statistically optimal noise properties, having tuned the algorithm to the specific CORE design and scanning strategy.

We have explored in detail the properties of the CORE noise maps, focusing for the sake of simplicity on a pair of detectors at $145 \mathrm{GHz}$, at positions in the centre and at the edges of the focal plane, to produce a triplet of $(I, Q, U)$ maps. We find that for all cases this set-up achieves full sky coverage and is able to cleanly separate the Stokes parameters, in the sense that the map-making equations are well-conditioned as measured by the reciprocal condition number of the covariance matrix of Stokes parameters in each pixel. This is a reassuring test, since an ill-conditioned mapmaking system is vulnerable to several systematic effects. Its outcome was not obvious beforehand: in the absence of an active hardware modulator such as a half-wave plate, the CORE instrument only relies on the spacecraft's scanning strategy to modulate the polarization signal. We have also verified that the pixel covariance matrices exhibit low intensity-to-polarization couplings, a desirable property that helps to keep intensity-to-polarization leakage low either by design, or under control with proper analysis tools. At the same time, residual $Q U$ couplings are not negligible and need to be accounted for during analysis. We have also attempted to optimize the scanning strategy, in particular the telescope offset and precession angles, finding that any advantage over the baseline configuration is minimal within a range compatible with reasonable assumptions about the spacecraft's design and operational constraints.

As a consequence of the very well interconnected scanning strategy, the CORE map-making achieves excellent levels of suppression of the correlated (' $1 / f$ ' type) noise component. We find that the residual correlation in the noise maps are in fact negligible for a detector knee frequency of the order of $10 \mathrm{mHz}$ and could easily be handled even for values a few times higher. We hence do not expect $1 / f$ noise to be a driver in the design and performance of the mission. Foreground contamination, which may leave hard to minimize residuals, is more of a concern on the largest angular scales, although it appears that the frequency coverage of CORE is adequate to guarantee a detection of $r \simeq 0.001$ even in the presence of complex foregrounds (Remazeilles et al. 2017).

We have also taken in consideration the case of noise correlations between detectors, such as those generated by a 'common mode' of thermal or other origin. Assuming a realistic model for this component (derived from Planck results), we find that a dedicated map-making scheme that takes such cross-correlations into account within the noise correction model would be beneficial for CORE (at the cost, however, of significantly increasing the computational burden of the problem, whose cost grows quadratically with the number of correlated detectors).

Since the CORE analysis uses multi-detector map-making (using a minimal set of two or four detectors as explored in this paper), bandpass mismatch leakage is a potential source of concern. Since there is no reason for the bandpass leakage systematic to be correlated between detectors, the effect is predicted to average out when increasing the number of detectors. In addition, we have implemented and demonstrated a correction scheme, which reduces the amount of leakage to a level well below the bounds on primordial $B B$ spectrum allowed by the CORE error budget.

Leakage from intensity to polarization arising due to beam asymmetries is a potential concern for high-precision CMB polarization experiment. The case of CORE requires even greater attention, due to the lack of active modulation in the optical path to regularize the beam shape independently of the scan. We have demonstrated two complementary approaches, in real space and harmonic space, 
that both allow to model accurately and quickly the impact of CORE beam non-idealities on the maps or the power spectra. When these simulations are employed to clean the CORE data, the uncorrected level of residuals is well within the CORE science requirements, even when accounting for imperfect knowledge of the instrument.

In an effort to move away from ideal simulations at the stage before map-making, we have implemented a prototype calibration pipeline for CORE, based on that used for Planck LFI. We have discussed its robustness to several non-idealities, arising due to the instrument and the sky, concluding that the calibration requirements of CORE can be already met with existing knowledge and algorithms.

Without doubt, an all-sky experiment to map the CMB polarization to cosmic variance level is an ambitious effort. Systematic contributions are expected to dominate the error budget and, if the mission is selected, a considerable effort will be necessary in the years ahead to build an analysis pipeline that accurately deals with them. While such a task will only be completed after critical information about the instrument will become available (that is, during the study phase and later), it would make sense to start building the necessary infrastructure as soon as possible. At the same time, it is reassuring that simple yet realistic assumptions about the main contaminants, implemented within quick and robust simulation and correction algorithms - all of which owe much to the heritage of Planck - demonstrate that our requirement to keep CORE systematics under control rests on solid grounds.

\section{Acknowledgments}

This research used resources of the National Energy Research Scientific Computing Center, which is supported by the Office of Science of the U.S. Department of Energy under Contract No. DE-AC0205CH11231. We also thank CSC - IT Center for Science (Finland) for computational resources. We acknowledge financial support by ASI Grant 2016-24-H.0 and Academy of Finland grant 295113. This work has received funding from the European Union's Horizon 2020 research and innovation programme under grant agreement number 687312. CJM is supported by an FCT Research Professorship, contract reference IF/00064/2012, funded by FCT/MCTES (Portugal) and POPH/FSE (EC). JGN acknowledges financial support from the Spanish MINECO for a 'Ramon y Cajal' fellowship (RYC-2013-13256) and the I+D 2015 project AYA2015-65887-P (MINECO/FEDER). GR acknowledges support from the National Research Foundation of Korea (NRF) through NRF-SGER 2014055950 funded by the Korean Ministry of Education, Science and Technology (MoEST), and from the faculty research fund of Sejong University in 2016. We thank Jean Kaplan for useful comments on the manuscript.

\section{Appendix A: Monte Carlo simulations}

In order to run TOAST to simulate one year of observations, we need a supercomputer. During the initial development of the CORE pipeline, making single runs and small sets of Monte Carlo realizations (10 at maximum), we used the high performance computing (HPC) resources at the National Energy Research Scientific Computing Center ${ }^{12}$ (NERSC) in the USA. In particular we used the Edison machine, a Cray XC30 supercomputer with 134,064 compute cores for a peak performance of 2.57 petaflop/s.

\footnotetext{
${ }^{12}$ http://www.nersc.gov
} 


\begin{tabular}{|c|c|c|}
\hline$f_{k}[\mathrm{mHz}]$ & CPU time [CPUh] & Memory footprint [GB] \\
\hline \multicolumn{3}{|c|}{ Boresight } \\
\hline 0 & 18320 & 586 \\
10 & 11580 & 586 \\
20 & 12280 & 586 \\
50 & 11730 & 586 \\
\hline \multicolumn{3}{|c|}{ High } \\
\hline 0 & 18270 & 570 \\
10 & 11480 & 570 \\
20 & 11260 & 570 \\
50 & 11950 & 570 \\
\hline \multicolumn{3}{|c|}{ Low } \\
\hline 0 & 18610 & 600 \\
10 & 11650 & 600 \\
50 & 11690 & 600 \\
\hline
\end{tabular}

Table 3. Resource consumption of the noise Monte Carlo cases

For the larger MC runs needed to derive the results shown in Sect. 4 and in particular the ones for the destriping tests in Sect. 4.3, we used the Centre for Scientific Computing (CSC) Sisu supercomputer ${ }^{13}$ in Finland. It is a Cray XC40 supercomputer with a total theoretical peak performance of 1.69 petaflop/s.

We considered 12 cases, generating 1000 realizations for each case. For each run we used 960 cores (40 nodes). We used four values of the knee frequency, $f_{k}=(0,10,20,50) \mathrm{mHz}$, and for each value of $f_{k}$ we generated maps for the 'boresight', 'high', and 'low' detector positions (see Table 1). In Table 10 we report the CPU and memory resources needed for each case. We saved the simulated noise, destriped and not, for all 1000 realizations, and the hit-map, the white noise covariance matrix and its inverse from the first realization (these are identical for all the realizations). The total size of these files is $3.4 \mathrm{~TB}$. The total computational cost of these simulations was $162100 \mathrm{CPU}$-hours.

\section{Appendix B: Algebra for cross correlation map-making}

In this Appendix we briefly review the algebra of the map-making procedure and derive the formalism adopted in Section 5. We suggest the interested reader refers to the references given in the text for a detailed discussion.

We model the observed data as follows:

$$
\mathbf{d}=\mathbf{A} \mathbf{m}+\mathbf{n},
$$

where the timelines of the $k$ detectors are combined:

$$
\mathbf{d} \equiv\left(\begin{array}{c}
\mathbf{d}^{(1)} \\
\vdots \\
\mathbf{d}^{(k)}
\end{array}\right),
$$

\footnotetext{
${ }^{13} \mathrm{http}: / /$ www.csc.fi
} 
and the generalized $k N_{d} \times 3 N_{p}$ pointing matrix becomes:

$$
\mathbf{A} \equiv\left(\begin{array}{ccc}
A_{t p}^{(1)} & A_{t p}^{(1)} \cos 2 \psi_{t}^{(1)} & A_{t p}^{(1)} \sin 2 \psi_{t}^{(1)} \\
\vdots & \vdots & \vdots \\
A_{t p}^{(k)} & A_{t p}^{(k)} \cos 2 \psi_{t}^{(k)} & A_{t p}^{(k)} \sin 2 \psi_{t}^{(k)}
\end{array}\right) .
$$

Here the $N_{d} \times N_{p}$ matrix $A_{t p}^{(j)}$ is the pointing matrix of the $j$-th detector, with elements equal to unity if the pixel $p$ is observed at time $t$ and zero otherwise. Each row of the pointing matrix is multiplied either by the cosine or the sine of $2 \psi_{t}$, where $\psi_{t}$ is the angle defining the polarimeter orientation at time $t$ with respect to the chosen reference frame. Similarly, the sky signal can be expressed as a $3 N_{p}$ vector:

$$
\mathbf{m} \equiv\left(\begin{array}{l}
\mathbf{I} \\
\mathbf{Q} \\
\mathbf{U}
\end{array}\right)
$$

where $\mathbf{I}, \mathbf{Q}$ and $\mathbf{U}$ are $N_{p}$ Stokes parameter vectors of the pixelized CMB sky. The noise timeline is:

$$
\mathbf{n} \equiv\left(\begin{array}{c}
\mathbf{n}^{(1)} \\
\vdots \\
\mathbf{n}^{(k)}
\end{array}\right) \text {. }
$$

where $\mathbf{n}^{(j)}$ is the $N_{d}$ element noise vector of the $j$-th detector, accounting for instrumental noise, atmospheric and temperature fluctuations, cosmic-ray hits and any other random systematic effect.

In the case of white noise, the generalized least squared (GLS) approach yields the following optimal estimator $\widetilde{\mathbf{m}}$ of $\mathbf{m}$ :

$$
\widetilde{\mathbf{m}}=\left(\mathbf{A}^{T} \mathbf{A}\right)^{-1} \mathbf{A}^{T} \mathbf{d} .
$$

This simply means to bin the samples in the map pixels. In the presence of correlated $1 / f$ noise, this approach, usually known as 'naive' map-making, leaves stripy structures in the map. Thus, the above formula is extended like follows:

$$
\widetilde{\mathbf{m}}=\left(\mathbf{A}^{T} \mathbf{N}^{-1} \mathbf{A}\right)^{-1} \mathbf{A}^{T} \mathbf{N}^{-1} \mathbf{d},
$$

where $\mathbf{N}$ is the noise covariance matrix $\left\langle\mathbf{n n}^{T}\right\rangle$. The matrix $\mathbf{N}$ is block-diagonal with respect to the detector index and each block can be inverted independently. Including also the cross-correlated noise among different detectors (i.e. the off-diagonal terms), the most general matrix $\mathbf{N}$ is given by:

$$
\mathbf{N} \equiv\left\langle\mathbf{n}_{t^{\prime}} \mathbf{n}_{t^{\prime}}\right\rangle=\left(\begin{array}{ccc}
\left\langle n_{t}^{(1)} n_{t^{\prime}}^{(1)}\right\rangle & \cdots & \left\langle n_{t}^{(1)} n_{t^{\prime}}^{(k)}\right\rangle \\
\vdots & \ddots & \vdots \\
\left\langle n_{t}^{(k)} n_{t^{\prime}}^{(1)}\right\rangle & \cdots & \left\langle n_{t}^{(k)} n_{t^{\prime}}^{(k)}\right\rangle
\end{array}\right),
$$

where $\langle\cdot\rangle$ denotes the expectation value. Some assumptions are made on the noise, in particular we assume that its statistical properties do not change over the mission life time (stationarity).

The stationarity property implies that $\mathbf{N}$ is a block-circulant matrix ${ }^{14}$ In this way, the inversion of $\mathbf{N}$ is much easier, since the Fourier counterpart of a block-circulant matrix is block-diagonal. Let us define the multichannel Fourier operator $\bar{F}$ such that:

$$
\overline{\mathbf{n}}=\bar{F} \mathbf{n},
$$

\footnotetext{
${ }^{14}$ Strictly speaking, stationarity implies that this matrix is block-Toeplitz, not block-circulant. Assuming it is circulant produces undesirable correlations between the end and the beginning of each block. This effect can be avoided by carefully zero padding the blocks before they are Fourier transformed.
} 
where:

$$
\overline{\mathbf{n}} \equiv\left(\begin{array}{c}
F \mathbf{n}^{(1)} \\
\vdots \\
F \mathbf{n}^{(k)}
\end{array}\right)
$$

contains end to end the Fourier transforms, $F \mathbf{n}^{(j)}$, of each segment of $\mathbf{n}$. Thus, in the case of a number of cross-correlated detectors, the inverse of $\mathbf{N}$ is given by:

$$
\mathbf{N}^{-1}=\bar{F}^{T} \mathbf{R}^{-1} \bar{F},
$$

where, under the assumption that the $N_{d} \times N_{d}$ matrix $\left\langle n_{t}^{(i)} n_{t^{\prime}}^{(j)}\right\rangle$ is circulant, $R^{(i j)}$ is a diagonal matrix with elements given by the noise cross-power spectrum between detectors $i$ and $j$ at frequency $f$ :

$$
R_{f f^{\prime}}^{(i j)}=P^{(i j)}(f) \delta_{f}^{f^{\prime}},
$$

To find $\widetilde{\mathbf{m}}$, the optimal GLS formula can be solved iteratively by the use of a Fourier-based, preconditioned conjugate gradient method.

To achieve a good convergence speed it is of paramount importance to provide a good preconditioner for the matrix $\left(\mathbf{A}^{T} \mathbf{N}^{-1} \mathbf{A}\right)$. Our choice is to approximate the matrix $\mathbf{N}^{-1}$ with its diagonal part and our preconditioner, $\mathbf{H}$, will be:

$$
\mathbf{H}=\left[\mathbf{A}^{T} \operatorname{diag}\left(\mathbf{N}^{-1}\right) \mathbf{A}\right]^{-1} .
$$

It can be shown that the preconditioning operator $\mathbf{H}$ is a $3 \times 3$ block diagonal matrix, where each block is the linear operator that solves for the three Stokes parameters of the given pixel (assuming white noise).

We define the inverse pixel condition number $\mathrm{R}_{\text {cond }}$ as the ratio of the absolute values of the smallest and largest eigenvalue of each block of $\mathbf{H}$. The condition number is a useful tool to trace the errors in the Stokes parameter estimation due to an inadequate polarization angle coverage on the given pixel. In particular $0 \leq \mathrm{R}_{\text {cond }} \leq 0.5$, assuming its lower value in the worst case and $R_{\text {cond }}=0.5$ in the limit of uniform angle coverage.

\section{References}

R. Banerji and J. Delabrouille. Evaluation and correction of the beam mismatch effects for future satellite CMB missions. In preparation, 2017.

E. J. Baxter, R. Keisler, S. Dodelson, K. A. Aird, S. W. Allen, M. L. N. Ashby, M. Bautz, M. Bayliss, B. A. Benson, L. E. Bleem, S. Bocquet, M. Brodwin, J. E. Carlstrom, C. L. Chang, I. Chiu, H.-M. Cho, A. Clocchiatti, T. M. Crawford, A. T. Crites, S. Desai, J. P. Dietrich, T. de Haan, M. A. Dobbs, R. J. Foley, W. R. Forman, E. M. George, M. D. Gladders, A. H. Gonzalez, N. W. Halverson, N. L. Harrington, C. Hennig, H. Hoekstra, G. P. Holder, W. L. Holzapfel, Z. Hou, J. D. Hrubes, C. Jones, L. Knox, A. T. Lee, E. M. Leitch, J. Liu, M. Lueker, D. Luong-Van, A. Mantz, D. P. Marrone, M. McDonald, J. J. McMahon, S. S. Meyer, M. Millea, L. M. Mocanu, S. S. Murray, S. Padin, C. Pryke, C. L. Reichardt, A. Rest, J. E. Ruhl, B. R. Saliwanchik, A. Saro, J. T. Sayre, K. K. Schaffer, E. Shirokoff, J. Song, H. G. Spieler, B. Stalder, S. A. Stanford, Z. Staniszewski, A. A. Stark, K. T. Story, A. van Engelen, K. Vanderlinde, J. D. Vieira, A. Vikhlinin, R. Williamson, O. Zahn, and A. Zenteno. A Measurement of Gravitational Lensing of the Cosmic Microwave Background by Galaxy Clusters Using Data from the South Pole Telescope. ApJ, 806:247, June 2015. doi: 10.1088/0004-637X/806/2/247. 
C. L. Bennett, D. Larson, J. L. Weiland, N. Jarosik, G. Hinshaw, N. Odegard, K. M. Smith, R. S. Hill, B. Gold, M. Halpern, E. Komatsu, M. R. Nolta, L. Page, D. N. Spergel, E. Wollack, J. Dunkley, A. Kogut, M. Limon, S. S. Meyer, G. S. Tucker, and E. L. Wright. Nine-year Wilkinson Microwave Anisotropy Probe (WMAP) Observations: Final Maps and Results. ApJS, 208:20, October 2013. doi: 10.1088/0067-0049/208/2/20.

BICEP2 Collaboration, Keck Array Collaboration, P. A. R. Ade, Z. Ahmed, R. W. Aikin, K. D. Alexander, D. Barkats, S. J. Benton, C. A. Bischoff, J. J. Bock, R. Bowens-Rubin, J. A. Brevik, I. Buder, E. Bullock, V. Buza, J. Connors, B. P. Crill, L. Duband, C. Dvorkin, J. P. Filippini, S. Fliescher, J. Grayson, M. Halpern, S. Harrison, G. C. Hilton, H. Hui, K. D. Irwin, K. S. Karkare, E. Karpel, J. P. Kaufman, B. G. Keating, S. Kefeli, S. A. Kernasovskiy, J. M. Kovac, C. L. Kuo, E. M. Leitch, M. Lueker, K. G. Megerian, C. B. Netterfield, H. T. Nguyen, R. O'Brient, R. W. Ogburn, A. Orlando, C. Pryke, S. Richter, R. Schwarz, C. D. Sheehy, Z. K. Staniszewski, B. Steinbach, R. V. Sudiwala, G. P. Teply, K. L. Thompson, J. E. Tolan, C. Tucker, A. D. Turner, A. G. Vieregg, A. C. Weber, D. V. Wiebe, J. Willmert, C. L. Wong, W. L. K. Wu, and K. W. Yoon. Improved Constraints on Cosmology and Foregrounds from BICEP2 and Keck Array Cosmic Microwave Background Data with Inclusion of 95 GHz Band. Physical Review Letters, 116(3): 031302, January 2016. doi: 10.1103/PhysRevLett.116.031302.

C. Burigana, M. Malaspina, N. Mandolesi, L. Danse, D. Maino, M. Bersanelli, and M. Maltoni. A preliminary study on destriping techniques of planck/lfi measurements versus observational strategy. ArXiv Astrophysics e-prints, June 1999.

CORE Collaboration, F. Finelli, M. Bucher, A. Achúcarro, M. Ballardini, N. Bartolo, D. Baumann, S. Clesse, J. Errard, W. Handley, M. Hindmarsh, K. Kiiveri, M. Kunz, A. Lasenby, M. Liguori, D. Paoletti, C. Ringeval, J. Väliviita, B. van Tent, V. Vennin, F. Arroja, M. Ashdown, A. J. Banday, R. Banerji, J. Baselmans, J. G. Bartlett, P. de Bernardis, M. Bersanelli, A. Bonaldi, J. Borril, F. R. Bouchet, F. Boulanger, T. Brinckmann, Z.-Y. Cai, M. Calvo, A. Challinor, J. Chluba, G. D'Amico, J. Delabrouille, J. María Diego, G. De Zotti, V. Desjacques, E. Di Valentino, S. Feeney, J. R. Fergusson, S. Ferraro, F. Forastieri, S. Galli, J. García-Bellido, R. T. Génova-Santos, M. Gerbino, J. González-Nuevo, S. Grandis, J. Greenslade, S. Hagstotz, S. Hanany, D. K. Hazra, C. Hernández-Monteagudo, E. Hivon, B. Hu, E. D. Kovetz, H. Kurki-Suonio, M. Lattanzi, J. Lesgourgues, J. Lizarraga, M. López-Caniego, G. Luzzi, B. Maffei, C. J. A. P. Martins, E. Martínez-González, D. McCarthy, S. Matarrese, A. Melchiorri, J.-B. Melin, A. Monfardini, P. Natoli, M. Negrello, F. Oppizzi, E. Pajer, S. P. Patil, M. Piat, G. Pisano, V. Poulin, A. Ravenni, M. Remazeilles, A. Renzi, D. Roest, L. Salvati, A. Tartari, G. Tasinato, J. Torrado, N. Trappe, M. Tucci, J. Urrestilla, P. Vielva, and R. Van de Weygaert. Exploring Cosmic Origins with CORE: Inflation. ArXiv e-prints, December 2016.

P. de Bernardis, P. A. R. Ade, J. J. A. Baselmans, E. S. Battistelli, A. Benoit, M. Bersanelli, A. Bideaud, M. Calvo, F. J. Casas, G. Castellano, A. Catalano, I. Charles, I. Colantoni, F. Columbro, A. Coppolecchia, M. Crook, G. D’Alessandro, M. De Petris, J. Delabrouille, S. Doyle, C. Franceschet, A. Gomez, J. Goupy, S. Hanany, M. Hills, L. Lamagna, J. Macias-Perez, B. Maffei, S. Martin, E. Martinez-Gonzalez, S. Masi, D. McCarthy, A. Mennella, A. Monfardini, F. Noviello, A. Paiella, F. Piacentini, M. Piat, G. Pisano, G. Signorelli, C. Y. Tan, A. Tartari, N. Trappe, S. Triqueneaux, C. Tucker, G. Vermeulen, K. Young, M. Zannoni, A. Achúcarro, R. Allison, M. Ashdown, M. Ballardini, A. J. Banday, R. Banerji, J. Bartlett, N. Bartolo, S. Basak, A. Bonaldi, M. Bonato, J. Borrill, F. Bouchet, F. Boulanger, T. Brinckmann, M. Bucher, C. Burigana, A. Buzzelli, Z. Y. Cai, C. S. Carvalho, A. Challinor, J. Chluba, S. Clesse, G. De Gasperis, G. De Zotti, E. Di Valentino, J. M. Diego, J. Errard, S. Feeney, R. Fernandez-Cobos, F. Finelli, F. Forastieri, S. Galli, R. Génova-Santos, M. Gerbino, J. González-Nuevo, S. Hagstotz, J. Greenslade, W. Handley, C. Hernández-Monteagudo, C. Hervias-Caimapo, E. Hivon, K. Kiiveri, T. Kisner, T. Kitching, M. Kunz, H. Kurki-Suonio, A. Lasenby, M. Lattanzi, J. Lesgourgues, A. Lewis, M. Liguori, V. Lindholm, G. Luzzi, C. J. A. P. Martins, A. Melchiorri, J. B. Melin, D. Molinari, P. Natoli, M. Negrello, A. Notari, D. Paoletti, G. Patanchon, L. Polastri, G. Polenta, A. Pollo, V. Poulin, M. Quartin, M. Remazeilles, M. Roman, J. A. Rubiño-Martín, L. Salvati, M. Tomasi, D. Tramonte, T. Trombetti, J. Väliviita, R. Van de Weijgaert, B. van Tent, V. Vennin, P. Vielva, N. Vittorio, and for the CORE collaboration. Exploring Cosmic Origins with CORE: The Instrument. ArXiv e-prints, May 2017. 
G. de Gasperis, A. Balbi, P. Cabella, P. Natoli, and N. Vittorio. ROMA: A map-making algorithm for polarised CMB data sets. A\&A, 436:1159-1165, June 2005. doi: 10.1051/0004-6361:20042512.

G. de Gasperis, A. Buzzelli, P. Cabella, P. de Bernardis, and N. Vittorio. Optimal cosmic microwave background map-making in the presence of cross-correlated noise. A\&A, 593:A15, August 2016. doi: $10.1051 / 0004-6361 / 201628143$

J. Delabrouille. Analysis of the accuracy of a destriping method for future cosmic microwave background mapping with the planck surveyor satellite. Astronomy and Astrophysics Supplement Series, 127(3): 555567, Feb 1998. ISSN 1286-4846. doi: 10.1051/aas:1998119. URL http://dx.doi.org/10.1051/aas:1998119.

J. Delabrouille, M. Betoule, J.-B. Melin, M.-A. Miville-Deschênes, J. Gonzalez-Nuevo, M. Le Jeune, G. Castex, G. de Zotti, S. Basak, M. Ashdown, J. Aumont, C. Baccigalupi, A. J. Banday, J.-P. Bernard, F. R. Bouchet, D. L. Clements, A. da Silva, C. Dickinson, F. Dodu, K. Dolag, F. Elsner, L. Fauvet, G. Faÿ, G. Giardino, S. Leach, J. Lesgourgues, M. Liguori, J. F. Macías-Pérez, M. Massardi, S. Matarrese, P. Mazzotta, L. Montier, S. Mottet, R. Paladini, B. Partridge, R. Piffaretti, G. Prezeau, S. Prunet, S. Ricciardi, M. Roman, B. Schaefer, and L. Toffolatti. The pre-launch Planck Sky Model: a model of sky emission at submillimetre to centimetre wavelengths. A\&A, 553:A96, May 2013. doi: $10.1051 / 0004-6361 / 201220019$.

J. Delabrouille, P. de Bernardis, F. R. Bouchet, A. Achúcarro, P. A. R. Ade, R. Allison, F. Arroja, E. Artal, M. Ashdown, C. Baccigalupi, M. Ballardini, A. J. Banday, R. Banerji, D. Barbosa, J. Bartlett, N. Bartolo, S. Basak, J. J. A. Baselmans, K. Basu, E. S. Battistelli, R. Battye, D. Baumann, A. Benoît, M. Bersanelli, A. Bideaud, M. Biesiada, M. Bilicki, A. Bonaldi, M. Bonato, J. Borrill, F. Boulanger, T. Brinckmann, M. L. Brown, M. Bucher, C. Burigana, A. Buzzelli, G. Cabass, Z.-Y. Cai, M. Calvo, A. Caputo, C.-S. Carvalho, F. J. Casas, G. Castellano, A. Catalano, A. Challinor, I. Charles, J. Chluba, D. L. Clements, S. Clesse, S. Colafrancesco, I. Colantoni, D. Contreras, A. Coppolecchia, M. Crook, G. D’Alessandro, G. D’Amico, A. da Silva, M. de Avillez, G. de Gasperis, M. De Petris, G. de Zotti, L. Danese, F.-X. Désert, V. Desjacques, E. Di Valentino, C. Dickinson, J. M. Diego, S. Doyle, R. Durrer, C. Dvorkin, H.-K. Eriksen, J. Errard, S. Feeney, R. Fernández-Cobos, F. Finelli, F. Forastieri, C. Franceschet, U. Fuskeland, S. Galli, R. T. Génova-Santos, M. Gerbino, E. Giusarma, A. Gomez, J. González-Nuevo, S. Grandis, J. Greenslade, J. Goupy, S. Hagstotz, S. Hanany, W. Handley, S. Henrot-Versillé, C. Hernández-Monteagudo, C. Hervias-Caimapo, M. Hills, M. Hindmarsh, E. Hivon, D. T. Hoang, D. C. Hooper, B. Hu, E. Keihänen, R. Keskitalo, K. Kiiveri, T. Kisner, T. Kitching, M. Kunz, H. Kurki-Suonio, G. Lagache, L. Lamagna, A. Lapi, A. Lasenby, M. Lattanzi, A. M. C. Le Brun, J. Lesgourgues, M. Liguori, V. Lindholm, J. Lizarraga, G. Luzzi, J. F. Macìas-Pérez, B. Maffei, N. Mandolesi, S. Martin, E. Martinez-Gonzalez, C. J. A. P. Martins, S. Masi, M. Massardi, S. Matarrese, P. Mazzotta, D. McCarthy, A. Melchiorri, J.-B. Melin, A. Mennella, J. Mohr, D. Molinari, A. Monfardini, L. Montier, P. Natoli, M. Negrello, A. Notari, F. Noviello, F. Oppizzi, C. O'Sullivan, L. Pagano, A. Paiella, E. Pajer, D. Paoletti, S. Paradiso, R. B. Partridge, G. Patanchon, S. P. Patil, O. Perdereau, F. Piacentini, M. Piat, G. Pisano, L. Polastri, G. Polenta, A. Pollo, N. Ponthieu, V. Poulin, D. Prêle, M. Quartin, A. Ravenni, M. Remazeilles, A. Renzi, C. Ringeval, D. Roest, M. Roman, B. F. Roukema, J.-A. Rubino-Martin, L. Salvati, D. Scott, S. Serjeant, G. Signorelli, A. A. Starobinsky, R. Sunyaev, C. Y. Tan, A. Tartari, G. Tasinato, L. Toffolatti, M. Tomasi, J. Torrado, D. Tramonte, N. Trappe, S. Triqueneaux, M. Tristram, T. Trombetti, M. Tucci, C. Tucker, J. Urrestilla, J. Väliviita, R. Van de Weygaert, B. Van Tent, V. Vennin, L. Verde, G. Vermeulen, P. Vielva, N. Vittorio, F. Voisin, C. Wallis, B. Wandelt, I. Wehus, J. Weller, K. Young, M. Zannoni, and for the CORE collaboration. Exploring Cosmic Origins with CORE: Survey requirements and mission design. ArXiv e-prints, June 2017.

E. Di Valentino, T. Brinckmann, M. Gerbino, V. Poulin, F. R. Bouchet, J. Lesgourgues, A. Melchiorri, J. Chluba, S. Clesse, J. Delabrouille, C. Dvorkin, F. Forastieri, S. Galli, D. C. Hooper, M. Lattanzi, C. J. A. P. Martins, L. Salvati, G. Cabass, A. Caputo, E. Giusarma, E. Hivon, P. Natoli, L. Pagano, S. Paradiso, J. A. Rubino-Martin, A. Achucarro, P. Ade, R. Allison, F. Arroja, M. Ashdown, M. Ballardini, A. J. Banday, R. Banerji, N. Bartolo, J. G. Bartlett, S. Basak, J. Baselmans, D. Baumann, P. de Bernardis, M. Bersanelli, A. Bonaldi, M. Bonato, J. Borrill, F. Boulanger, M. Bucher, C. Burigana, A. Buzzelli, Z.-Y. 
Cai, M. Calvo, C. S. Carvalho, G. Castellano, A. Challinor, I. Charles, I. Colantoni, A. Coppolecchia, M. Crook, G. D’Alessandro, M. De Petris, G. De Zotti, J. M. Diego, J. Errard, S. Feeney,

R. Fernandez-Cobos, S. Ferraro, F. Finelli, G. de Gasperis, R. T. Génova-Santos, J. González-Nuevo,

S. Grandis, J. Greenslade, S. Hagstotz, S. Hanany, W. Handley, D. K. Hazra, C. Hernández-Monteagudo,

C. Hervias-Caimapo, M. Hills, K. Kiiveri, T. Kisner, T. Kitching, M. Kunz, H. Kurki-Suonio, L. Lamagna, A. Lasenby, A. Lewis, M. Liguori, V. Lindholm, M. Lopez-Caniego, G. Luzzi, B. Maffei, S. Martin, E. Martinez-Gonzalez, S. Masi, D. McCarthy, J.-B. Melin, J. J. Mohr, D. Molinari, A. Monfardini, M. Negrello, A. Notari, A. Paiella, D. Paoletti, G. Patanchon, F. Piacentini, M. Piat, G. Pisano, L. Polastri, G. Polenta, A. Pollo, M. Quartin, M. Remazeilles, M. Roman, C. Ringeval, A. Tartari, M. Tomasi, D. Tramonte, N. Trappe, T. Trombetti, C. Tucker, J. Väliviita, R. van de Weygaert, B. Van Tent, V. Vennin, G. Vermeulen, P. Vielva, N. Vittorio, K. Young, M. Zannoni, and for the CORE collaboration. Exploring Cosmic Origins with CORE: Cosmological Parameters. ArXiv e-prints, November 2016.

O. Doré, R. Teyssier, F. R. Bouchet, D. Vibert, and S. Prunet. MAPCUMBA: A fast iterative multi-grid map-making algorithm for CMB experiments. A\&A, 374:358-370, July 2001. doi: 10.1051/0004-6361:20010692.

K. M. Górski, E. Hivon, A. J. Banday, B. D. Wandelt, F. K. Hansen, M. Reinecke, and M. Bartelmann. HEALPix: A Framework for High-Resolution Discretization and Fast Analysis of Data Distributed on the Sphere. ApJ, 622:759-771, April 2005. doi: 10.1086/427976.

L. M. Griffiths and C. H. Lineweaver. Testing the Cosmic Microwave Background Data for Systematic Effects. ApJ, 603:371-382, March 2004. doi: 10.1086/381493.

E. Hivon, S. Mottet, and N. Ponthieu. Quickpol: Fast calculation of effective beam matrices for CMB polarization. A\&A, 598:A25, February 2017. URL http://dx.doi.org/10.1051/0004-6361/201629626.

D.T. Hoang, G. Patanchon, M. Bucher, T. Matsumura, R. Banerji, H. Ishino, M. Hazumi, and J. Delabrouille. Evaluation of the effect of bandpass mismatch for future satellite CMB missions. In preparation, 2017.

J. Kaplan and J. Delabrouille. Some sources of systematic errors on CMB polarized measurements with bolometers. In S. Cecchini, S. Cortiglioni, R. Sault, and C. Sbarra, editors, Astrophysical Polarized Backgrounds, volume 609 of American Institute of Physics Conference Series, pages 209-214, March 2002. doi: $10.1063 / 1.1471849$.

A. Karakci, L. Zhang, P. M. Sutter, E. F. Bunn, A. Korotkov, P. Timbie, G. S. Tucker, and B. D. Wandelt. Systematic Effects in Interferometric Observations of the Cosmic Microwave Background Polarization. ApJS, 207:14, July 2013. doi: 10.1088/0067-0049/207/1/14.

E. Keihänen, H. Kurki-Suonio, T. Poutanen, D. Maino, and C. Burigana. A maximum likelihood approach to the destriping technique. AEFA, 428:287-298, December 2004. doi: 10.1051/0004-6361:200400060.

E. Keihänen, H. Kurki-Suonio, and T. Poutanen. MADAM- a map-making method for CMB experiments. MNRAS, 360:390-400, June 2005. doi: 10.1111/j.1365-2966.2005.09055.x.

E. Keihänen, R. Keskitalo, H. Kurki-Suonio, T. Poutanen, and A.-S. Sirviö. Making cosmic microwave background temperature and polarization maps with MADAM. A\&A, 510:A57, February 2010. doi: 10.1051/0004-6361/200912813.

A. Kosowsky. Introduction to microwave background polarization. New Astr. Rev., 43:157-168, July 1999. doi: 10.1016/S1387-6473(99)00009-3.

H. Kurki-Suonio, E. Keihänen, R. Keskitalo, T. Poutanen, A.-S. Sirviö, D. Maino, and C. Burigana. Destriping CMB temperature and polarization maps. AEAA, 506:1511-1539, November 2009. doi: 10.1051/0004-6361/200912361.

C. H. Lineweaver, G. F. Smoot, C. L. Bennett, E. L. Wright, L. Tenorio, A. Kogut, P. B. Keegstra, G. Hinshaw, and A. J. Banday. Correlated noise in the COBE DMR sky maps. ApJ, 436:452-455, December 1994. doi: 10.1086/174920. 
T. Louis, E. Grace, M. Hasselfield, M. Lungu, L. Maurin, G. E. Addison, P. A. R. Ade, S. Aiola, R. Allison, M. Amiri, E. Angile, N. Battaglia, J. A. Beall, F. de Bernardis, J. R. Bond, J. Britton, E. Calabrese, H.-m. Cho, S. K. Choi, K. Coughlin, D. Crichton, K. Crowley, R. Datta, M. J. Devlin, S. R. Dicker, J. Dunkley, R. Dünner, S. Ferraro, A. E. Fox, P. Gallardo, M. Gralla, M. Halpern, S. Henderson, J. C. Hill, G. C. Hilton, M. Hilton, A. D. Hincks, R. Hlozek, S. P. P. Ho, Z. Huang, J. Hubmayr, K. M. Huffenberger, J. P. Hughes, L. Infante, K. Irwin, S. Muya Kasanda, J. Klein, B. Koopman, A. Kosowsky, D. Li,

M. Madhavacheril, T. A. Marriage, J. McMahon, F. Menanteau, K. Moodley, C. Munson, S. Naess, F. Nati, L. Newburgh, J. Nibarger, M. D. Niemack, M. R. Nolta, C. Nuñez, L. A. Page, C. Pappas, B. Partridge, F. Rojas, E. Schaan, B. L. Schmitt, N. Sehgal, B. D. Sherwin, J. Sievers, S. Simon, D. N. Spergel, S. T. Staggs, E. R. Switzer, R. Thornton, H. Trac, J. Treu, C. Tucker, A. Van Engelen, J. T. Ward, and E. J. Wollack. The Atacama Cosmology Telescope: Two-Season ACTPol Spectra and Parameters. ArXiv e-prints, October 2016.

D. Maino, C. Burigana, M. Maltoni, B. D. Wandelt, K. M. Grski, M. Malaspina, M. Bersanelli, N. Mandolesi, A. J. Banday, and E. Hivon. The Planck-lfi instrument: Analysis of the 1/f noise and implications for the scanning strategy. Astron. Astrophys. Suppl. Ser., 140(3):383391, Dec 1999. ISSN 1286-4846. doi: 10.1051/aas:1999429. URL http://dx. doi . org/10.1051/aas : 1999429.

A. Mangilli, S. Plaszczynski, and M. Tristram. Large-scale cosmic microwave background temperature and polarization cross-spectra likelihoods. MNRAS, 453:3174-3189, November 2015. doi: 10.1093/mnras/stv1733.

S. Masi, P. A. R. Ade, J. J. Bock, J. R. Bond, J. Borrill, A. Boscaleri, P. Cabella, C. R. Contaldi, B. P. Crill, P. de Bernardis, G. de Gasperis, A. de Oliveira-Costa, G. de Troia, G. di Stefano, P. Ehlers, E. Hivon, V. Hristov, A. Iacoangeli, A. H. Jaffe, W. C. Jones, T. S. Kisner, A. E. Lange, C. J. MacTavish, C. Marini Bettolo, P. Mason, P. D. Mauskopf, T. E. Montroy, F. Nati, L. Nati, P. Natoli, C. B. Netterfield, E. Pascale, F. Piacentini, D. Pogosyan, G. Polenta, S. Prunet, S. Ricciardi, G. Romeo, J. E. Ruhl, P. Santini, M. Tegmark, E. Torbet, M. Veneziani, and N. Vittorio. Instrument, method, brightness, and polarization maps from the 2003 flight of BOOMERanG. A\&A, 458:687-716, November 2006. doi: 10.1051/0004-6361:20053891.

J. C. Mather, D. J. Fixsen, R. A. Shafer, C. Mosier, and D. T. Wilkinson. Calibrator design for the cobe far infrared absolute spectrophotometer (firas). ApJ, 512(2):511, 1999. URL http: //stacks . iop.org/0004-637X/512/i=2/a=511.

N. J. Miller, M. Shimon, and B. G. Keating. CMB beam systematics: Impact on lensing parameter estimation. Phys.Rev.D, 79(6):063008, March 2009a. doi: 10.1103/PhysRevD.79.063008.

N. J. Miller, M. Shimon, and B. G. Keating. CMB polarization systematics due to beam asymmetry: Impact on cosmological birefringence. Phys.Rev.D, 79(10):103002, May 2009b. doi: 10.1103/PhysRevD.79.103002.

P. Natoli, G. de Gasperis, C. Gheller, and N. Vittorio. A Map-Making algorithm for the Planck Surveyor. A\&A, 372:346-356, June 2001. doi: 10.1051/0004-6361:20010393.

P. Natoli, D. Marinucci, P. Cabella, G. de Gasperis, and N. Vittorio. Non-iterative methods to estimate the in-flight noise properties of CMB detectors. A\&A, 383:1100-1112, March 2002. doi: 10.1051/0004-6361:20011788.

L. Pagano, P. de Bernardis, G. de Troia, G. Gubitosi, S. Masi, A. Melchiorri, P. Natoli, F. Piacentini, and G. Polenta. CMB polarization systematics, cosmological birefringence, and the gravitational waves background. Phys.Rev.D, 80(4):043522, August 2009. doi: 10.1103/PhysRevD.80.043522.

G. Patanchon, P. A. R. Ade, J. J. Bock, E. L. Chapin, M. J. Devlin, S. Dicker, M. Griffin, J. O. Gundersen, M. Halpern, P. C. Hargrave, D. H. Hughes, J. Klein, G. Marsden, P. G. Martin, P. Mauskopf, C. B. Netterfield, L. Olmi, E. Pascale, M. Rex, D. Scott, C. Semisch, M. D. P. Truch, C. Tucker, G. S. Tucker, M. P. Viero, and D. V. Wiebe. SANEPIC: A Mapmaking Method for Time Stream Data from Large Arrays. ApJ, 681:708-725, July 2008. doi: 10.1086/588543. 
Planck Collaboration, Ade, P. A. R., Aghanim, N., Armitage-Caplan, C., Arnaud, M., Ashdown, M., Atrio-Barandela, F., Aumont, J., Baccigalupi, C., Banday, A. J., Barreiro, R. B., Bartlett, J. G., Battaner, E., Benabed, K., Benot, A., Benoit-Lvy, A., Bernard, J.-P., Bersanelli, M., Bielewicz, P., Bobin, J., Bock, J. J., Bonaldi, A., Bonavera, L., Bond, J. R., Borrill, J., Bouchet, F. R., Boulanger, F., Bridges, M., Bucher, M., Burigana, C., Butler, R. C., Calabrese, E., Cardoso, J.-F., Catalano, A., Challinor, A., Chamballu, A., Chiang, H. C., Chiang, L.-Y, Christensen, P. R., Church, S., Clements, D. L., Colombi, S., Colombo, L. P. L., Combet, C., Couchot, F., Coulais, A., Crill, B. P., Curto, A., Cuttaia, F., Danese, L., Davies, R. D., Davis, R. J., de Bernardis, P., de Rosa, A., de Zotti, G., Delabrouille, J., Delouis, J.-M., Dsert, F.-X., Dickinson, C., Diego, J. M., Dole, H., Donzelli, S., Dor, O., Douspis, M., Dunkley, J., Dupac, X., Efstathiou, G., Elsner, F., Enlin, T. A., Eriksen, H. K., Finelli, F., Forni, O., Frailis, M., Fraisse, A. A., Franceschi, E., Gaier, T. C., Galeotta, S., Galli, S., Ganga, K., Giard, M., Giardino, G., Giraud-Hraud, Y., Gjerlw, E., Gonzlez-Nuevo, J., Grski, K. M., Gratton, S., Gregorio, A., Gruppuso, A., Gudmundsson, J. E., Hansen, F. K., Hanson, D., Harrison, D., Helou, G., Henrot-Versill, S., Hernndez-Monteagudo, C., Herranz, D., Hildebrandt, S. R., Hivon, E., Hobson, M., Holmes, W. A., Hornstrup, A., Hovest, W., Huffenberger, K. M., Hurier, G., Jaffe, A. H., Jaffe, T. R., Jewell, J., Jones, W. C., Juvela, M., Keihnen, E., Keskitalo, R., Kiiveri, K., Kisner, T. S., Kneissl, R., Knoche, J., Knox, L., Kunz, M., Kurki-Suonio, H., Lagache, G., Lhteenmki, A., Lamarre, J.-M., Lasenby, A., Lattanzi, M., Laureijs, R. J., Lawrence, C. R., Le Jeune, M., Leach, S., Leahy, J. P., Leonardi, R., Len-Tavares, J., Lesgourgues, J., Liguori, M., Lilje, P. B., Linden-Vrnle, M., Lindholm, V., Lpez-Caniego, M., Lubin, P. M., Macas-Prez, J. F., Maffei, B., Maino, D., Mandolesi, N., Marinucci, D., Maris, M., Marshall, D. J., Martin, P. G., Martnez-Gonzlez, E., Masi, S., Massardi, M., Matarrese, S., Matthai, F., Mazzotta, P., Meinhold, P. R., Melchiorri, A., Mendes, L., Menegoni, E., Mennella, A., Migliaccio, M., Millea, M., Mitra, S., Miville-Deschłnes, M.-A., Molinari, D., Moneti, A., Montier, L., Morgante, G., Mortlock, D., Moss, A., Munshi, D., Murphy, J. A., Naselsky, P., Nati, F., Natoli, P., Netterfield, C. B., Nrgaard-Nielsen, H. U., Noviello, F., Novikov, D., Novikov, I., ODwyer, I. J., Orieux, F., Osborne, S., Oxborrow, C. A., Paci, F., Pagano, L., Pajot, F., Paladini, R., Paoletti, D., Partridge, B., Pasian, F., Patanchon, G., Paykari, P., Perdereau, O., Perotto, L., Perrotta, F., Piacentini, F., Piat, M., Pierpaoli, E., Pietrobon, D., Plaszczynski, S., Pointecouteau, E., Polenta, G., Ponthieu, N., Popa, L., Poutanen, T., Pratt, G. W., Przeau, G., Prunet, S., Puget, J.-L., Rachen, J. P., Rahlin, A., Rebolo, R., Reinecke, M., Remazeilles, M., Renault, C., Ricciardi, S., Riller, T., Ringeval, C., Ristorcelli, I., Rocha, G., Rosset, C., Roudier, G., Rowan-Robinson, M., Rubio-Martn, J. A., Rusholme, B., Sandri, M., Sanselme, L., Santos, D., Savini, G., Scott, D., Seiffert, M. D., Shellard, E. P. S., Spencer, L. D., Starck, J.-L., Stolyarov, V., Stompor, R., Sudiwala, R., Sureau, F., Sutton, D., Suur-Uski, A.-S., Sygnet, J.-F., Tauber, J. A., Tavagnacco, D., Terenzi, L., Toffolatti, L., Tomasi, M., Tristram, M., Tucci, M., Tuovinen, J., Trler, M., Valenziano, L., Valiviita, J., Van Tent, B., Varis, J., Vielva, P., Villa, F., Vittorio, N., Wade, L. A., Wandelt, B. D., Wehus, I. K., White, M., White, S. D. M., Yvon, D., Zacchei, A., and Zonca, A. Planck 2013 results. XV. CMB power spectra and likelihood. A\&A, 571:A15, November 2014a. doi: 10.1051/0004-6361/201321573.

Planck Collaboration, Ade, P. A. R., Aghanim, N., Armitage-Caplan, C., Arnaud, M., Ashdown, M., Atrio-Barandela, F., Aumont, J., Baccigalupi, C., Banday, A. J., Barreiro, R. B., Battaner, E., Benabed, K., Benot, A., Benoit-Lvy, A., Bernard, J.-P., Bersanelli, M., Bertincourt, B., Bielewicz, P., Bobin, J., Bock, J. J., Bond, J. R., Borrill, J., Bouchet, F. R., Boulanger, F., Bridges, M., Bucher, M., Burigana, C., Cardoso, J.-F., Catalano, A., Challinor, A., Chamballu, A., Chary, R.-R., Chen, X., Chiang, H. C., Chiang, L.-Y, Christensen, P. R., Church, S., Clements, D. L., Colombi, S., Colombo, L. P. L., Combet, C., Couchot, F., Coulais, A., Crill, B. P., Curto, A., Cuttaia, F., Danese, L., Davies, R. D., de Bernardis, P., de Rosa, A., de Zotti, G., Delabrouille, J., Delouis, J.-M., Dsert, F.-X., Dickinson, C., Diego, J. M., Dole, H., Donzelli, S., Dor, O., Douspis, M., Dupac, X., Efstathiou, G., Enlin, T. A., Eriksen, H. K., Filliard, C., Finelli, F., Forni, O., Frailis, M., Franceschi, E., Galeotta, S., Ganga, K., Giard, M., Giardino, G., Giraud-Hraud, Y., Gonzlez-Nuevo, J., Grski, K. M., Gratton, S., Gregorio, A., Gruppuso, A., Hansen, F. K., Hanson, D., Harrison, D., Helou, G., Henrot-Versill, S., Hernndez-Monteagudo, C., Herranz, D., Hildebrandt, S. R., Hivon, E., Hobson, M., Holmes, W. A., Hornstrup, A., Hovest, W., Huffenberger, K. M., Jaffe, A. H., Jaffe, T. R., Jones, W. C., Juvela, M., Keihnen, E., Keskitalo, R., Kisner, T. S., Kneissl, R., Knoche, J., Knox, L., Kunz, M., Kurki-Suonio, H., Lagache, G., Lamarre, J.-M., Lasenby, A., Laureijs, R. J., Lawrence, C. R., Le Jeune, M., Lellouch, E., Leonardi, R., Leroy, C., Lesgourgues, J., Liguori, M., Lilje, P. B., 
Linden-Vrnle, M., Lpez-Caniego, M., Lubin, P. M., Macas-Prez, J. F., Maffei, B., Mandolesi, N., Maris, M., Marshall, D. J., Martin, P. G., Martnez-Gonzlez, E., Masi, S., Massardi, M., Matarrese, S., Matthai, F., Maurin, L., Mazzotta, P., McGehee, P., Meinhold, P. R., Melchiorri, A., Mendes, L., Mennella, A., Migliaccio, M., Mitra, S., Miville-Deschłnes, M.-A., Moneti, A., Montier, L., Moreno, R., Morgante, G., Mortlock, D., Munshi, D., Murphy, J. A., Naselsky, P., Nati, F., Natoli, P., Netterfield, C. B., Nrgaard-Nielsen, H. U., Noviello, F., Novikov, D., Novikov, I., Osborne, S., Oxborrow, C. A., Paci, F., Pagano, L., Pajot, F., Paladini, R., Paoletti, D., Partridge, B., Pasian, F., Patanchon, G., Pearson, T. J., Perdereau, O., Perotto, L., Perrotta, F., Piacentini, F., Piat, M., Pierpaoli, E., Pietrobon, D., Plaszczynski, S., Pointecouteau, E., Polenta, G., Ponthieu, N., Popa, L., Poutanen, T., Pratt, G. W., Przeau, G., Prunet, S., Puget, J.-L., Rachen, J. P., Reinecke, M., Remazeilles, M., Renault, C., Ricciardi, S., Riller, T., Ristorcelli, I., Rocha, G., Rosset, C., Roudier, G., Rusholme, B., Santos, D., Savini, G., Scott, D., Shellard, E. P. S., Spencer, L. D., Starck, J.-L., Stolyarov, V., Stompor, R., Sudiwala, R., Sunyaev, R., Sureau, F., Sutton, D., Suur-Uski, A.-S., Sygnet, J.-F., Tauber, J. A., Tavagnacco, D., Techene, S., Terenzi, L., Tomasi, M., Tristram, M., Tucci, M., Umana, G., Valenziano, L., Valiviita, J., Van Tent, B., Vielva, P., Villa, F., Vittorio, N., Wade, L. A., Wandelt, B. D., Yvon, D., Zacchei, A., and Zonca, A. Planck 2013 results. viii. hfi photometric calibration and mapmaking. AEFA, 571:A8, 2014b. doi: 10.1051/0004-6361/201321538. URL http: //dx . doi .org/10.1051/0004-6361/201321538.

Planck Collaboration, Adam, R., Ade, P. A. R., Aghanim, N., Arnaud, M., Ashdown, M., Aumont, J., Baccigalupi, C., Banday, A. J., Barreiro, R. B., Bartolo, N., Battaner, E., Benabed, K., Benot, A., Benoit-Lvy, A., Bernard, J.-P., Bersanelli, M., Bertincourt, B., Bielewicz, P., Bock, J. J., Bonavera, L., Bond, J. R., Borrill, J., Bouchet, F. R., Boulanger, F., Bucher, M., Burigana, C., Calabrese, E., Cardoso, J.-F., Catalano, A., Challinor, A., Chamballu, A., Chiang, H. C., Christensen, P. R., Clements, D. L., Colombi, S., Colombo, L. P. L., Combet, C., Couchot, F., Coulais, A., Crill, B. P., Curto, A., Cuttaia, F., Danese, L., Davies, R. D., Davis, R. J., de Bernardis, P., de Rosa, A., de Zotti, G., Delabrouille, J., Delouis, J.-M., Dsert, F.-X., Diego, J. M., Dole, H., Donzelli, S., Dor, O., Douspis, M., Ducout, A., Dupac, X., Efstathiou, G., Elsner, F., Enlin, T. A., Eriksen, H. K., Falgarone, E., Fergusson, J., Finelli, F., Forni, O., Frailis, M., Fraisse, A. A., Franceschi, E., Frejsel, A., Galeotta, S., Galli, S., Ganga, K., Ghosh, T., Giard, M., Giraud-Hraud, Y., Gjerlw, E., Gonzlez-Nuevo, J., Grski, K. M., Gratton, S., Gruppuso, A., Gudmundsson, J. E., Hansen, F. K., Hanson, D., Harrison, D. L., Henrot-Versill, S., Herranz, D., Hildebrandt, S. R., Hivon, E., Hobson, M., Holmes, W. A., Hornstrup, A., Hovest, W., Huffenberger, K. M., Hurier, G., Jaffe, A. H., Jaffe, T. R., Jones, W. C., Juvela, M., Keihnen, E., Keskitalo, R., Kisner, T. S., Kneissl, R., Knoche, J., Kunz, M., Kurki-Suonio, H., Lagache, G., Lamarre, J.-M., Lasenby, A., Lattanzi, M., Lawrence, C. R., Le Jeune, M., Leahy, J. P., Lellouch, E., Leonardi, R., Lesgourgues, J., Levrier, F., Liguori, M., Lilje, P. B., Linden-Vrnle, M., Lpez-Caniego, M., Lubin, P. M., Macas-Prez, J. F., Maggio, G., Maino, D., Mandolesi, N., Mangilli, A., Maris, M., Martin, P. G., Martnez-Gonzlez, E., Masi, S., Matarrese, S., McGehee, P., Melchiorri, A., Mendes, L., Mennella, A., Migliaccio, M., Mitra, S., Miville-Deschłnes, M.-A., Moneti, A., Montier, L., Moreno, R., Morgante, G., Mortlock, D., Moss, A., Mottet, S., Munshi, D., Murphy, J. A., Naselsky, P., Nati, F., Natoli, P., Netterfield, C. B., Nrgaard-Nielsen, H. U., Noviello, F., Novikov, D., Novikov, I., Oxborrow, C. A., Paci, F., Pagano, L., Pajot, F., Paoletti, D., Pasian, F., Patanchon, G., Pearson, T. J., Perdereau, O., Perotto, L., Perrotta, F., Pettorino, V., Piacentini, F., Piat, M., Pierpaoli, E., Pietrobon, D., Plaszczynski, S., Pointecouteau, E., Polenta, G., Pratt, G. W., Przeau, G., Prunet, S., Puget, J.-L., Rachen, J. P., Reinecke, M., Remazeilles, M., Renault, C., Renzi, A.,

Ristorcelli, I., Rocha, G., Rosset, C., Rossetti, M., Roudier, G., Rusholme, B., Sandri, M., Santos, D., Sauv, A., Savelainen, M., Savini, G., Scott, D., Seiffert, M. D., Shellard, E. P. S., Spencer, L. D., Stolyarov, V., Stompor, R., Sudiwala, R., Sutton, D., Suur-Uski, A.-S., Sygnet, J.-F., Tauber, J. A., Terenzi, L., Toffolatti, L., Tomasi, M., Tristram, M., Tucci, M., Tuovinen, J., Valenziano, L., Valiviita, J., Van Tent, B., Vibert, L., Vielva, P., Villa, F., Wade, L. A., Wandelt, B. D., Watson, R., Wehus, I. K., Yvon, D., Zacchei, A., and Zonca, A. Planck 2015 results. viii. high frequency instrument data processing: Calibration and maps. AEFA, 594:A8, 2015a. doi: 10.1051/0004-6361/201525820. URL http: //dx . doi .org/10.1051/0004-6361/201525820.

Planck Collaboration, Ade, P. A. R., Aghanim, N., Ashdown, M., Aumont, J., Baccigalupi, C., Banday, A. J., Barreiro, R. B., Bartolo, N., Battaglia, P., Battaner, E., Benabed, K., Benot, A., Benoit-Lvy, A., Bernard, 
J.-P., Bersanelli, M., Bielewicz, P., Bock, J. J., Bonaldi, A., Bonavera, L., Bond, J. R., Borrill, J., Bouchet, F. R., Bucher, M., Burigana, C., Butler, R. C., Calabrese, E., Cardoso, J.-F., Catalano, A., Chamballu, A., Christensen, P. R., Colombi, S., Colombo, L. P. L., Crill, B. P., Curto, A., Cuttaia, F., Danese, L., Davies, R. D., Davis, R. J., de Bernardis, P., de Rosa, A., de Zotti, G., Delabrouille, J., Dickinson, C., Diego, J. M., Dole, H., Donzelli, S., Dor, O., Douspis, M., Ducout, A., Dupac, X., Efstathiou, G., Elsner, F., Enlin, T. A., Eriksen, H. K., Fergusson, J., Finelli, F., Forni, O., Frailis, M., Franceschi, E., Frejsel, A., Galeotta, S.,

Galli, S., Ganga, K., Giard, M., Giraud-Hraud, Y., Gjerlw, E., Gonzlez-Nuevo, J., Grski, K. M., Gratton, S., Gregorio, A., Gruppuso, A., Hansen, F. K., Hanson, D., Harrison, D. L., Henrot-Versill, S., Herranz, D., Hildebrandt, S. R., Hivon, E., Hobson, M., Holmes, W. A., Hornstrup, A., Hovest, W., Huffenberger, K. M., Hurier, G., Jaffe, A. H., Jaffe, T. R., Juvela, M., Keihnen, E., Keskitalo, R., Kisner, T. S., Knoche, J., Krachmalnicoff, N., Kunz, M., Kurki-Suonio, H., Lagache, G., Lhteenmki, A., Lamarre, J.-M., Lasenby, A., Lattanzi, M., Lawrence, C. R., Leahy, J. P., Leonardi, R., Lesgourgues, J., Levrier, F., Liguori, M., Lilje, P. B., Linden-Vrnle, M., Lpez-Caniego, M., Lubin, P. M., Macas-Prez, J. F., Maggio, G., Maino, D., Mandolesi, N., Mangilli, A., Maris, M., Martin, P. G., Martnez-Gonzlez, E., Masi, S., Matarrese, S., McGehee, P., Meinhold, P. R., Melchiorri, A., Mendes, L., Mennella, A., Migliaccio, M., Mitra, S., Montier, L., Morgante, G., Mortlock, D., Moss, A., Munshi, D., Murphy, J. A., Naselsky, P., Nati, F., Natoli, P., Netterfield, C. B., Nrgaard-Nielsen, H. U., Novikov, D., Novikov, I., Paci, F., Pagano, L., Pajot, F., Paoletti, D., Partridge, B., Pasian, F., Patanchon, G., Pearson, T. J., Peel, M., Perdereau, O., Perotto, L., Perrotta, F., Pettorino, V., Piacentini, F., Pierpaoli, E., Pietrobon, D., Pointecouteau, E., Polenta, G., Pratt, G. W., Przeau, G., Prunet, S., Puget, J.-L., Rachen, J. P., Rebolo, R., Reinecke, M., Remazeilles, M., Renzi, A., Rocha, G., Romelli, E., Rosset, C., Rossetti, M., Roudier, G., Rubio-Martn, J. A., Rusholme, B., Sandri, M., Santos, D., Savelainen, M., Scott, D., Seiffert, M. D., Shellard, E. P. S., Spencer, L. D., Stolyarov, V., Sutton, D., Suur-Uski, A.-S., Sygnet, J.-F., Tauber, J. A., Tavagnacco, D., Terenzi, L., Toffolatti, L., Tomasi, M., Tristram, M., Tucci, M., Tuovinen, J., Trler, M., Umana, G., Valenziano, L., Valiviita, J., Van Tent, B., Vassallo, T., Vielva, P., Villa, F., Wade, L. A., Wandelt, B. D., Watson, R., Wehus, I. K., Wilkinson, A., Yvon, D., Zacchei, A., and Zonca, A. Planck 2015 results. v. lfi calibration. AEEA, 594:A5, 2015b. doi: 10.1051/0004-6361/201526632. URL http://dx . doi .org/10. 1051/0004-6361/201526632.

Planck Collaboration, Adam, R., Ade, P. A. R., Aghanim, N., Akrami, Y., Alves, M. I. R., Argeso, F., Arnaud, M., Arroja, F., Ashdown, M., Aumont, J., Baccigalupi, C., Ballardini, M., Banday, A. J., Barreiro, R. B., Bartlett, J. G., Bartolo, N., Basak, S., Battaglia, P., Battaner, E., Battye, R., Benabed, K., Benot, A., Benoit-Lvy, A., Bernard, J.-P., Bersanelli, M., Bertincourt, B., Bielewicz, P., Bikmaev, I., Bock, J. J., Bhringer, H., Bonaldi, A., Bonavera, L., Bond, J. R., Borrill, J., Bouchet, F. R., Boulanger, F., Bucher, M., Burenin, R., Burigana, C., Butler, R. C., Calabrese, E., Cardoso, J.-F., Carvalho, P., Casaponsa, B., Castex, G., Catalano, A., Challinor, A., Chamballu, A., Chary, R.-R., Chiang, H. C., Chluba, J., Chon, G., Christensen, P. R., Church, S., Clemens, M., Clements, D. L., Colombi, S., Colombo, L. P. L., Combet, C., Comis, B., Contreras, D., Couchot, F., Coulais, A., Crill, B. P., Cruz, M., Curto, A., Cuttaia, F., Danese, L., Davies, R. D., Davis, R. J., de Bernardis, P., de Rosa, A., de Zotti, G., Delabrouille, J., Delouis, J.-M., Dsert, F.-X., Di Valentino, E., Dickinson, C., Diego, J. M., Dolag, K., Dole, H., Donzelli, S., Dor, O., Douspis, M., Ducout, A., Dunkley, J., Dupac, X., Efstathiou, G., Eisenhardt, P. R. M., Elsner, F., Enlin, T. A., Eriksen, H. K., Falgarone, E., Fantaye, Y., Farhang, M., Feeney, S., Fergusson, J., Fernandez-Cobos, R., Feroz, F., Finelli, F., Florido, E., Forni, O., Frailis, M., Fraisse, A. A., Franceschet, C., Franceschi, E., Frejsel, A., Frolov, A., Galeotta, S., Galli, S., Ganga, K., Gauthier, C., Gnova-Santos, R. T., Gerbino, M., Ghosh, T., Giard, M., Giraud-Hraud, Y., Giusarma, E., Gjerlw, E., Gonzlez-Nuevo, J., Grski, K. M., Grainge, K. J. B., Gratton, S., Gregorio, A., Gruppuso, A., Gudmundsson, J. E., Hamann, J., Handley, W., Hansen, F. K., Hanson, D., Harrison, D. L., Heavens, A., Helou, G., Henrot-Versill, S.,

Hernndez-Monteagudo, C., Herranz, D., Hildebrandt, S. R., Hivon, E., Hobson, M., Holmes, W. A., Hornstrup, A., Hovest, W., Huang, Z., Huffenberger, K. M., Hurier, G., Ili, S., Jaffe, A. H., Jaffe, T. R., Jin, T., Jones, W. C., Juvela, M., Karakci, A., Keihnen, E., Keskitalo, R., Khamitov, I., Kiiveri, K., Kim, J., Kisner, T. S., Kneissl, R., Knoche, J., Knox, L., Krachmalnicoff, N., Kunz, M., Kurki-Suonio, H., Lacasa, F., Lagache, G., Lhteenmki, A., Lamarre, J.-M., Langer, M., Lasenby, A., Lattanzi, M., Lawrence, C. R., Le Jeune, M., Leahy, J. P., Lellouch, E., Leonardi, R., Len-Tavares, J., Lesgourgues, J., Levrier, F., Lewis, A., Liguori, M., Lilje, P. B., Lilley, M., Linden-Vrnle, M., Lindholm, V., Liu, H., Lpez-Caniego, M., Lubin, P. M., Ma, Y.-Z., Macas-Prez, J. F., Maggio, G., Maino, D., Mak, D. S. Y., Mandolesi, N., Mangilli, A., 
Marchini, A., Marcos-Caballero, A., Marinucci, D., Maris, M., Marshall, D. J., Martin, P. G., Martinelli, M., Martnez-Gonzlez, E., Masi, S., Matarrese, S., Mazzotta, P., McEwen, J. D., McGehee, P., Mei, S., Meinhold, P. R., Melchiorri, A., Melin, J.-B., Mendes, L., Mennella, A., Migliaccio, M., Mikkelsen, K., Millea, M., Mitra, S., Miville-Deschłnes, M.-A., Molinari, D., Moneti, A., Montier, L., Moreno, R., Morgante, G., Mortlock, D., Moss, A., Mottet, S., Mnchmeyer, M., Munshi, D., Murphy, J. A., Narimani, A., Naselsky, P., Nastasi, A., Nati, F., Natoli, P., Negrello, M., Netterfield, C. B., Nrgaard-Nielsen, H. U., Noviello, F., Novikov, D., Novikov, I., Olamaie, M., Oppermann, N., Orlando, E., Oxborrow, C. A., Paci, F., Pagano, L., Pajot, F., Paladini, R., Pandolfi, S., Paoletti, D., Partridge, B., Pasian, F., Patanchon, G., Pearson, T. J., Peel, M., Peiris, H. V., Pelkonen, V.-M., Perdereau, O., Perotto, L., Perrott, Y. C., Perrotta, F., Pettorino, V., Piacentini, F., Piat, M., Pierpaoli, E., Pietrobon, D., Plaszczynski, S., Pogosyan, D., Pointecouteau, E., Polenta, G., Popa, L., Pratt, G. W., Przeau, G., Prunet, S., Puget, J.-L., Rachen, J. P., Racine, B., Reach, W. T., Rebolo, R., Reinecke, M., Remazeilles, M., Renault, C., Renzi, A., Ristorcelli, I., Rocha, G., Roman, M., Romelli, E., Rosset, C., Rossetti, M., Rotti, A., Roudier, G., Rouill dOrfeuil, B., Rowan-Robinson, M., Rubio-Martn, J. A., Ruiz-Granados, B., Rumsey, C., Rusholme, B., Said, N., Salvatelli, V., Salvati, L., Sandri, M., Sanghera, H. S., Santos, D., Saunders, R. D. E., Sauv, A., Savelainen, M., Savini, G., Schaefer, B. M., Schammel, M. P., Scott, D., Seiffert, M. D., Serra, P., Shellard, E. P. S., Shimwell, T. W., Shiraishi, M., Smith, K., Souradeep, T., Spencer, L. D., Spinelli, M., Stanford, S. A., Stern, D., Stolyarov, V., Stompor, R., Strong, A. W., Sudiwala, R., Sunyaev, R., Sutter, P., Sutton, D., Suur-Uski, A.-S., Sygnet, J.-F., Tauber, J. A., Tavagnacco, D., Terenzi, L., Texier, D., Toffolatti, L., Tomasi, M., Tornikoski, M., Tramonte, D., Tristram, M., Troja, A., Trombetti, T., Tucci, M., Tuovinen, J., Trler, M., Umana, G., Valenziano, L., Valiviita, J., Van Tent, F., Vassallo, T., Vibert, L., Vidal, M., Viel, M., Vielva, P., Villa, F., Wade, L. A., Walter, B., Wandelt, B. D., Watson, R., Wehus, I. K., Welikala, N., Weller, J., White, M., White, S. D. M., Wilkinson, A., Yvon, D., Zacchei, A., Zibin, J. P., and Zonca, A. Planck 2015 results - i. overview of products and scientific results. AEFA, 594:A1, 2016a. doi: 10.1051/0004-6361/201527101. URL http://dx . doi .org/10.1051/0004-6361/201527101.

Planck Collaboration, Adam, R., Ade, P. A. R., Aghanim, N., Alves, M. I. R., Arnaud, M., Ashdown, M., Aumont, J., Baccigalupi, C., Banday, A. J., Barreiro, R. B., Bartlett, J. G., Bartolo, N., Battaner, E., Benabed, K., Benot, A., Benoit-Lvy, A., Bernard, J.-P., Bersanelli, M., Bielewicz, P., Bock, J. J., Bonaldi, A., Bonavera, L., Bond, J. R., Borrill, J., Bouchet, F. R., Boulanger, F., Bucher, M., Burigana, C., Butler, R. C., Calabrese, E., Cardoso, J.-F., Catalano, A., Challinor, A., Chamballu, A., Chary, R.-R., Chiang, H. C., Christensen, P. R., Clements, D. L., Colombi, S., Colombo, L. P. L., Combet, C., Couchot, F., Coulais, A., Crill, B. P., Curto, A., Cuttaia, F., Danese, L., Davies, R. D., Davis, R. J., de Bernardis, P., de Rosa, A., de Zotti, G., Delabrouille, J., Dsert, F.-X., Dickinson, C., Diego, J. M., Dole, H., Donzelli, S., Dor, O., Douspis, M., Ducout, A., Dupac, X., Efstathiou, G., Elsner, F., Enlin, T. A., Eriksen, H. K., Falgarone, E., Fergusson, J., Finelli, F., Forni, O., Frailis, M., Fraisse, A. A., Franceschi, E., Frejsel, A., Galeotta, S., Galli, S., Ganga, K., Ghosh, T., Giard, M., Giraud-Hraud, Y., Gjerlw, E., Gonzlez-Nuevo, J., Grski, K. M., Gratton, S., Gregorio, A., Gruppuso, A., Gudmundsson, J. E., Hansen, F. K., Hanson, D., Harrison, D. L., Helou, G., Henrot-Versill, S., Hernndez-Monteagudo, C., Herranz, D., Hildebrandt, S. R., Hivon, E., Hobson, M., Holmes, W. A., Hornstrup, A., Hovest, W., Huffenberger, K. M., Hurier, G., Jaffe, A. H., Jaffe, T. R., Jones, W. C., Juvela, M., Keihnen, E., Keskitalo, R., Kisner, T. S., Kneissl, R., Knoche, J., Kunz, M., Kurki-Suonio, H., Lagache, G., Lhteenmki, A., Lamarre, J.-M., Lasenby, A., Lattanzi, M., Lawrence, C. R., Le Jeune, M., Leahy, J. P., Leonardi, R., Lesgourgues, J., Levrier, F., Liguori, M., Lilje, P. B., Linden-Vrnle, M., Lpez-Caniego, M., Lubin, P. M., Macas-Prez, J. F., Maggio, G., Maino, D., Mandolesi, N., Mangilli, A., Maris, M., Marshall, D. J., Martin, P. G., Martnez-Gonzlez, E., Masi, S., Matarrese, S., McGehee, P., Meinhold, P. R., Melchiorri, A., Mendes, L., Mennella, A., Migliaccio, M., Mitra, S., Miville-Deschłnes, M.-A., Moneti, A., Montier, L., Morgante, G., Mortlock, D., Moss, A., Munshi, D., Murphy, J. A., Naselsky, P., Nati, F., Natoli, P., Netterfield, C. B., Nrgaard-Nielsen, H. U., Noviello, F., Novikov, D., Novikov, I., Orlando, E., Oxborrow, C. A., Paci, F., Pagano, L., Pajot, F., Paladini, R., Paoletti, D., Partridge, B., Pasian, F., Patanchon, G., Pearson, T. J., Perdereau, O., Perotto, L., Perrotta, F., Pettorino, V., Piacentini, F., Piat, M., Pierpaoli, E., Pietrobon, D., Plaszczynski, S., Pointecouteau, E., Polenta, G., Pratt, G. W., Przeau, G., Prunet, S., Puget, J.-L., Rachen, J. P., Reach, W. T., Rebolo, R., Reinecke, M., Remazeilles, M., Renault, C., Renzi, A., Ristorcelli, I., Rocha, G., Rosset, C., Rossetti, M., Roudier, G., Rubio-Martn, J. A., Rusholme, B., Sandri, M., Santos, D., Savelainen, M., Savini, G., Scott, D., Seiffert, M. 
D., Shellard, E. P. S., Spencer, L. D., Stolyarov, V., Stompor, R., Strong, A. W., Sudiwala, R., Sunyaev, R., Sutton, D., Suur-Uski, A.-S., Sygnet, J.-F., Tauber, J. A., Terenzi, L., Toffolatti, L., Tomasi, M., Tristram, M., Tucci, M., Tuovinen, J., Umana, G., Valenziano, L., Valiviita, J., Van Tent, F., Vielva, P., Villa, F., Wade, L. A., Wandelt, B. D., Wehus, I. K., Wilkinson, A., Yvon, D., Zacchei, A., and Zonca, A. Planck 2015 results. X. Diffuse component separation: Foreground maps. A\&A, 594:A10, September 2016 b.

Planck Collaboration, Adam, R., Ade, P. A. R., Aghanim, N., Arnaud, M., Ashdown, M., Aumont, J., Baccigalupi, C., Banday, A. J., Barreiro, R. B., Bartolo, N., Battaner, E., Benabed, K., Benot, A., Benoit-Lvy, A., Bernard, J.-P., Bersanelli, M., Bertincourt, B., Bielewicz, P., Bock, J. J., Bonavera, L., Bond, J. R., Borrill, J., Bouchet, F. R., Boulanger, F., Bucher, M., Burigana, C., Calabrese, E., Cardoso, J.-F., Catalano, A., Challinor, A., Chamballu, A., Chary, R.-R., Chiang, H. C., Christensen, P. R., Clements, D. L., Colombi, S., Colombo, L. P. L., Combet, C., Couchot, F., Coulais, A., Crill, B. P., Curto, A., Cuttaia, F., Danese, L., Davies, R. D., Davis, R. J., de Bernardis, P., de Rosa, A., de Zotti, G., Delabrouille, J., Delouis, J.-M., Dsert, F.-X., Diego, J. M., Dole, H., Donzelli, S., Dor, O., Douspis, M., Ducout, A., Dupac, X., Efstathiou, G., Elsner, F., Enlin, T. A., Eriksen, H. K., Falgarone, E., Fergusson, J., Finelli, F., Forni, O., Frailis, M., Fraisse, A. A., Franceschi, E., Frejsel, A., Galeotta, S., Galli, S., Ganga, K., Ghosh, T., Giard, M., Giraud-Hraud, Y., Gjerlw, E., Gonzlez-Nuevo, J., Grski, K. M., Gratton, S., Gruppuso, A., Gudmundsson, J. E., Hansen, F. K., Hanson, D., Harrison, D. L., Henrot-Versill, S., Herranz, D., Hildebrandt, S. R., Hivon, E., Hobson, M., Holmes, W. A., Hornstrup, A., Hovest, W., Huffenberger, K. M., Hurier, G., Jaffe, A. H., Jaffe, T. R., Jones, W. C., Juvela, M., Keihnen, E., Keskitalo, R., Kisner, T. S., Kneissl, R., Knoche, J., Kunz, M., Kurki-Suonio, H., Lagache, G., Lamarre, J.-M., Lasenby, A., Lattanzi, M., Lawrence, C. R., Le Jeune, M., Leahy, J. P., Lellouch, E., Leonardi, R., Lesgourgues, J., Levrier, F., Liguori, M., Lilje, P. B., Linden-Vrnle, M., Lpez-Caniego, M., Lubin, P. M., Macas-Prez, J. F., Maggio, G., Maino, D., Mandolesi, N., Mangilli, A., Maris, M., Martin, P. G., Martnez-Gonzlez, E., Masi, S., Matarrese, S., McGehee, P., Melchiorri, A., Mendes, L., Mennella, A., Migliaccio, M., Mitra, S., Miville-Deschłnes, M.-A., Moneti, A., Montier, L., Moreno, R., Morgante, G., Mortlock, D., Moss, A., Mottet, S., Munshi, D., Murphy, J. A., Naselsky, P., Nati, F., Natoli, P., Netterfield, C. B., Nrgaard-Nielsen, H. U., Noviello, F., Novikov, D., Novikov, I., Oxborrow, C. A., Paci, F., Pagano, L., Pajot, F., Paoletti, D., Pasian, F., Patanchon, G., Pearson, T. J., Perdereau, O., Perotto, L., Perrotta, F., Pettorino, V., Piacentini, F., Piat, M., Pierpaoli, E., Pietrobon, D., Plaszczynski, S., Pointecouteau, E., Polenta, G., Pratt, G. W., Przeau, G., Prunet, S., Puget, J.-L., Rachen, J. P., Reinecke, M., Remazeilles, M., Renault, C., Renzi, A.,

Ristorcelli, I., Rocha, G., Rosset, C., Rossetti, M., Roudier, G., Rowan-Robinson, M., Rusholme, B., Sandri, M., Santos, D., Sauv, A., Savelainen, M., Savini, G., Scott, D., Seiffert, M. D., Shellard, E. P. S., Spencer, L. D., Stolyarov, V., Stompor, R., Sudiwala, R., Sutton, D., Suur-Uski, A.-S., Sygnet, J.-F., Tauber, J. A., Terenzi, L., Toffolatti, L., Tomasi, M., Tristram, M., Tucci, M., Tuovinen, J., Valenziano, L., Valiviita, J., Van Tent, B., Vibert, L., Vielva, P., Villa, F., Wade, L. A., Wandelt, B. D., Watson, R., Wehus, I. K., Yvon, D., Zacchei, A., and Zonca, A. Planck 2015 results. VII. High Frequency Instrument data processing: Time-ordered information and beams. A\&A, 594:A7, September 2016c. doi: 10.1051/0004-6361/201525844.

Planck Collaboration, P. A. R. Ade, J. Aumont, C. Baccigalupi, A. J. Banday, R. B. Barreiro, N. Bartolo, S. Basak, P. Battaglia, E. Battaner, K. Benabed, A. Benoit-Lévy, J.-P. Bernard, M. Bersanelli, P. Bielewicz, A. Bonaldi, L. Bonavera, J. R. Bond, J. Borrill, C. Burigana, R. C. Butler, E. Calabrese, A. Catalano, P. R. Christensen, L. P. L. Colombo, M. Cruz, A. Curto, F. Cuttaia, L. Danese, R. D. Davies, R. J. Davis, P. de Bernardis, A. de Rosa, G. de Zotti, J. Delabrouille, C. Dickinson, J. M. Diego, O. Doré, A. Ducout, X. Dupac, F. Elsner, T. A. Enßlin, H. K. Eriksen, F. Finelli, M. Frailis, C. Franceschet, E. Franceschi, S. Galeotta, S. Galli, K. Ganga, T. Ghosh, M. Giard, Y. Giraud-Héraud, E. Gjerløw, J. González-Nuevo, K. M. Górski, A. Gregorio, A. Gruppuso, F. K. Hansen, D. L. Harrison, C. Hernández-Monteagudo, D. Herranz, S. R. Hildebrandt, E. Hivon, M. Hobson, A. Hornstrup, W. Hovest, K. M. Huffenberger, G. Hurier, A. H. Jaffe, T. R. Jaffe, E. Keihänen, R. Keskitalo, K. Kiiveri, T. S. Kisner, J. Knoche, N. Krachmalnicoff, M. Kunz, H. Kurki-Suonio, G. Lagache, J.-M. Lamarre, A. Lasenby, M. Lattanzi, C. R. Lawrence, J. P. Leahy, R. Leonardi, F. Levrier, M. Liguori, P. B. Lilje, M. Linden-Vørnle, V. Lindholm, M. López-Caniego, P. M. Lubin, J. F. Macías-Pérez, B. Maffei, G. Maggio, D. Maino, N. Mandolesi, A. Mangilli, M. Maris, P. G. Martin, E. Martínez-González, S. Masi, S. Matarrese, P. R. Meinhold, 
A. Mennella, M. Migliaccio, S. Mitra, L. Montier, G. Morgante, D. Mortlock, D. Munshi, J. A. Murphy, F. Nati, P. Natoli, F. Noviello, F. Paci, L. Pagano, F. Pajot, D. Paoletti, B. Partridge, F. Pasian, T. J. Pearson, O. Perdereau, V. Pettorino, F. Piacentini, E. Pointecouteau, G. Polenta, G. W. Pratt, J.-L. Puget, J. P. Rachen, M. Reinecke, M. Remazeilles, A. Renzi, I. Ristorcelli, G. Rocha, C. Rosset, M. Rossetti, G. Roudier, J. A. Rubiño-Martín, B. Rusholme, M. Sandri, D. Santos, M. Savelainen, D. Scott, V. Stolyarov, R. Stompor, A.-S. Suur-Uski, J.-F. Sygnet, J. A. Tauber, D. Tavagnacco, L. Terenzi, L. Toffolatti, M. Tomasi, M. Tristram, M. Tucci, G. Umana, L. Valenziano, J. Valiviita, B. Van Tent, T. Vassallo, P. Vielva, F. Villa, L. A. Wade, B. D. Wandelt, R. Watson, I. K. Wehus, D. Yvon, A. Zacchei, J. P. Zibin, and A. Zonca. Planck 2015 results. III. LFI systematic uncertainties. A\&A, 594:A3, August 2016d. doi: 10.1051/0004-6361/201526998.

Planck Collaboration, Ade, P. A. R., Aghanim, N., Arnaud, M., Ashdown, M., Aumont, J., Baccigalupi, C., Banday, A. J., Barreiro, R. B., Bartlett, J. G., Bartolo, N., Battaner, E., Benabed, K., Benot, A., Benoit-Lvy, A., Bernard, J.-P., Bersanelli, M., Bielewicz, P., Bock, J. J., Bonaldi, A., Bonavera, L., Bond, J. R., Borrill, J., Bouchet, F. R., Boulanger, F., Bucher, M., Burigana, C., Butler, R. C., Calabrese, E., Cardoso, J.-F., Castex, G., Catalano, A., Challinor, A., Chamballu, A., Chiang, H. C., Christensen, P. R., Clements, D. L., Colombi, S., Colombo, L. P. L., Combet, C., Couchot, F., Coulais, A., Crill, B. P., Curto, A., Cuttaia, F., Danese, L., Davies, R. D., Davis, R. J., de Bernardis, P., de Rosa, A., de Zotti, G., Delabrouille, J., Delouis, J.-M., Dsert, F.-X., Dickinson, C., Diego, J. M., Dolag, K., Dole, H., Donzelli, S., Dor, O., Douspis, M., Ducout, A., Dupac, X., Efstathiou, G., Elsner, F., Enlin, T. A., Eriksen, H. K., Fergusson, J., Finelli, F., Forni, O., Frailis, M., Fraisse, A. A., Franceschi, E., Frejsel, A., Galeotta, S., Galli, S., Ganga, K., Ghosh, T., Giard, M., Giraud-Hraud, Y., Gjerlw, E., Gonzlez-Nuevo, J., Grski, K. M., Gratton, S., Gregorio, A., Gruppuso, A., Gudmundsson, J. E., Hansen, F. K., Hanson, D., Harrison, D. L., Henrot-Versill, S., Hernndez-Monteagudo, C., Herranz, D., Hildebrandt, S. R., Hivon, E., Hobson, M., Holmes, W. A., Hornstrup, A., Hovest, W., Huffenberger, K. M., Hurier, G., Jaffe, A. H., Jaffe, T. R., Jones, W. C., Juvela, M., Karakci, A., Keihnen, E., Keskitalo, R., Kiiveri, K., Kisner, T. S., Kneissl, R., Knoche, J., Kunz, M., Kurki-Suonio, H., Lagache, G., Lamarre, J.-M., Lasenby, A., Lattanzi, M., Lawrence, C. R., Leonardi, R., Lesgourgues, J., Levrier, F., Liguori, M., Lilje, P. B., Linden-Vrnle, M., Lindholm, V., Lpez-Caniego, M., Lubin, P. M., Macas-Prez, J. F., Maggio, G., Maino, D., Mandolesi, N., Mangilli, A., Maris, M., Martin, P. G., Martnez-Gonzlez, E., Masi, S., Matarrese, S., McGehee, P., Meinhold, P. R., Melchiorri, A., Melin, J.-B., Mendes, L., Mennella, A., Migliaccio, M., Mitra, S., Miville-Deschłnes, M.-A., Moneti, A., Montier, L., Morgante, G., Mortlock, D., Moss, A., Munshi, D., Murphy, J. A., Naselsky, P., Nati, F., Natoli, P., Netterfield, C. B., Nrgaard-Nielsen, H. U., Noviello, F., Novikov, D., Novikov, I., Oxborrow, C. A., Paci, F., Pagano, L., Pajot, F., Paoletti, D., Pasian, F., Patanchon, G., Pearson, T. J., Perdereau, O., Perotto, L., Perrotta, F., Pettorino, V., Piacentini, F., Piat, M., Pierpaoli, E., Pietrobon, D., Plaszczynski, S., Pointecouteau, E., Polenta, G., Pratt, G. W., Przeau, G., Prunet, S., Puget, J.-L., Rachen, J. P., Rebolo, R., Reinecke, M., Remazeilles, M., Renault, C., Renzi, A., Ristorcelli, I., Rocha, G., Roman, M., Rosset, C., Rossetti, M., Roudier, G., Rubio-Martn, J. A., Rusholme, B., Sandri, M., Santos, D., Savelainen, M., Scott, D., Seiffert, M. D., Shellard, E. P. S., Spencer, L. D., Stolyarov, V., Stompor, R., Sudiwala, R., Sutton, D., Suur-Uski, A.-S., Sygnet, J.-F., Tauber, J. A., Terenzi, L., Toffolatti, L., Tomasi, M., Tristram, M., Tucci, M., Tuovinen, J., Valenziano, L., Valiviita, J., Van Tent, B., Vielva, P., Villa, F., Wade, L. A., Wandelt, B. D., Wehus, I. K., Welikala, N., Yvon, D., Zacchei, A., and Zonca, A. Planck 2015 results. XII. Full focal plane simulations. A\&A, 594:A12, September 2016e. doi: 10.1051/0004-6361/201527103.

Planck Collaboration, Ade, P. A. R., Aghanim, N., Ashdown, M., Aumont, J., Baccigalupi, C., Ballardini, M., Banday, A. J., Barreiro, R. B., Bartolo, N., Basak, S., Battaglia, P., Battaner, E., Benabed, K., Benot, A., Benoit-Lvy, A., Bernard, J.-P., Bersanelli, M., Bielewicz, P., Bock, J. J., Bonaldi, A., Bonavera, L., Bond, J. R., Borrill, J., Bouchet, F. R., Bucher, M., Burigana, C., Butler, R. C., Calabrese, E., Cardoso, J.-F., Castex, G., Catalano, A., Chamballu, A., Christensen, P. R., Colombi, S., Colombo, L. P. L., Crill, B. P., Curto, A., Cuttaia, F., Danese, L., Davies, R. D., Davis, R. J., de Bernardis, P., de Rosa, A., de Zotti, G., Delabrouille, J., Dickinson, C., Diego, J. M., Dole, H., Donzelli, S., Dor, O., Douspis, M., Ducout, A., Dupac, X., Efstathiou, G., Elsner, F., Enlin, T. A., Eriksen, H. K., Fergusson, J., Finelli, F., Forni, O., Frailis, M., Franceschet, C., Franceschi, E., Frejsel, A., Galeotta, S., Galli, S., Ganga, K., Giard, M., Giraud-Hraud, Y., Gjerlw, E., Gonzlez-Nuevo, J., Grski, K. M., Gratton, S., Gregorio, A., Gruppuso, A., Hansen, F. K., 
Hanson, D., Harrison, D. L., Henrot-Versill, S., Herranz, D., Hildebrandt, S. R., Hivon, E., Hobson, M., Holmes, W. A., Hornstrup, A., Hovest, W., Huffenberger, K. M., Hurier, G., Jaffe, A. H., Jaffe, T. R., Juvela, M., Keihnen, E., Keskitalo, R., Kiiveri, K., Kisner, T. S., Knoche, J., Krachmalnicoff, N., Kunz, M., Kurki-Suonio, H., Lagache, G., Lhteenmki, A., Lamarre, J.-M., Lasenby, A., Lattanzi, M., Lawrence, C. R., Leahy, J. P., Leonardi, R., Lesgourgues, J., Levrier, F., Liguori, M., Lilje, P. B., Linden-Vrnle, M., Lindholm, V., Lpez-Caniego, M., Lubin, P. M., Macas-Prez, J. F., Maggio, G., Maino, D., Mandolesi, N., Mangilli, A., Maris, M., Martin, P. G., Martnez-Gonzlez, E., Masi, S., Matarrese, S., Mazzotta, P., McGehee, P., Meinhold, P. R., Melchiorri, A., Mendes, L., Mennella, A., Migliaccio, M., Mitra, S., Montier, L., Morgante, G., Morisset, N., Mortlock, D., Moss, A., Munshi, D., Murphy, J. A., Naselsky, P., Nati, F., Natoli, P., Netterfield, C. B., Nrgaard-Nielsen, H. U., Novikov, D., Novikov, I., Oppermann, N., Paci, F., Pagano, L., Paoletti, D., Partridge, B., Pasian, F., Patanchon, G., Pearson, T. J., Peel, M., Perdereau, O., Perotto, L., Perrotta, F., Pettorino, V., Piacentini, F., Pierpaoli, E., Pietrobon, D., Pointecouteau, E., Polenta, G., Pratt, G. W., Przeau, G., Prunet, S., Puget, J.-L., Rachen, J. P., Rebolo, R., Reinecke, M., Remazeilles, M., Renzi, A., Rocha, G., Romelli, E., Rosset, C., Rossetti, M., Roudier, G., Rubio-Martn, J. A., Rusholme, B., Sandri, M., Santos, D., Savelainen, M., Scott, D., Seiffert, M. D., Shellard, E. P. S., Spencer, L. D., Stolyarov, V., Sutton, D., Suur-Uski, A.-S., Sygnet, J.-F., Tauber, J. A., Tavagnacco, D., Terenzi, L., Toffolatti, L., Tomasi, M., Tristram, M., Tucci, M., Tuovinen, J., Trler, M., Umana, G., Valenziano, L., Valiviita, J., Van Tent, B., Vassallo, T., Vielva, P., Villa, F., Wade, L. A., Wandelt, B. D., Watson, R., Wehus, I. K., Wilkinson, A., Yvon, D., Zacchei, A., and Zonca, A. Planck 2015 results. II. Low Frequency Instrument data processings. A\&A, 594:A2, September 2016f. doi: 10.1051/0004-6361/201525818

Planck Collaboration, N. Aghanim, M. Ashdown, J. Aumont, C. Baccigalupi, M. Ballardini, A. J. Banday, R. B. Barreiro, N. Bartolo, S. Basak, R. Battye, K. Benabed, J.-P. Bernard, M. Bersanelli, P. Bielewicz, J. J. Bock, A. Bonaldi, L. Bonavera, J. R. Bond, J. Borrill, F. R. Bouchet, F. Boulanger, M. Bucher, C. Burigana, R. C. Butler, E. Calabrese, J.-F. Cardoso, J. Carron, A. Challinor, H. C. Chiang, L. P. L. Colombo, C. Combet, B. Comis, A. Coulais, B. P. Crill, A. Curto, F. Cuttaia, R. J. Davis, P. de Bernardis, A. de Rosa, G. de Zotti, J. Delabrouille, J.-M. Delouis, E. Di Valentino, C. Dickinson, J. M. Diego, O. Doré, M. Douspis, A. Ducout, X. Dupac, G. Efstathiou, F. Elsner, T. A. Enßlin, H. K. Eriksen, E. Falgarone, Y. Fantaye, F. Finelli, F. Forastieri, M. Frailis, A. A. Fraisse, E. Franceschi, A. Frolov, S. Galeotta, S. Galli, K. Ganga, R. T. Génova-Santos, M. Gerbino, T. Ghosh, J. González-Nuevo, K. M. Górski, S. Gratton, A. Gruppuso, J. E. Gudmundsson, F. K. Hansen, G. Helou, S. Henrot-Versillé, D. Herranz, E. Hivon, Z. Huang, S. Ilić, A. H. Jaffe, W. C. Jones, E. Keihänen, R. Keskitalo, T. S. Kisner, L. Knox, N. Krachmalnicoff, M. Kunz, H. Kurki-Suonio, G. Lagache, J.-M. Lamarre, M. Langer, A. Lasenby, M. Lattanzi, C. R. Lawrence, M. Le Jeune, J. P. Leahy, F. Levrier, M. Liguori, P. B. Lilje, M. López-Caniego, Y.-Z. Ma, J. F. Macías-Pérez, G. Maggio, A. Mangilli, M. Maris, P. G. Martin, E. Martínez-González, S. Matarrese, N. Mauri, J. D. McEwen, P. R. Meinhold, A. Melchiorri, A. Mennella, M. Migliaccio, M.-A. Miville-Deschênes, D. Molinari, A. Moneti, L. Montier, G. Morgante, A. Moss, S. Mottet, P. Naselsky, P. Natoli, C. A. Oxborrow, L. Pagano, D. Paoletti, B. Partridge, G. Patanchon, L. Patrizii, O. Perdereau, L. Perotto, V. Pettorino, F. Piacentini, S. Plaszczynski, L. Polastri, G. Polenta, J.-L. Puget, J. P. Rachen, B. Racine, M. Reinecke, M. Remazeilles, A. Renzi, G. Rocha, M. Rossetti, G. Roudier, J. A. Rubiño-Martín, B. Ruiz-Granados, L. Salvati, M. Sandri, M. Savelainen, D. Scott, G. Sirri, R. Sunyaev, A.-S. Suur-Uski, J. A. Tauber, M. Tenti, L. Toffolatti, M. Tomasi, M. Tristram, T. Trombetti, J. Valiviita, F. Van Tent, L. Vibert, P. Vielva, F. Villa, N. Vittorio, B. D. Wandelt, R. Watson, I. K. Wehus, M. White, A. Zacchei, and A. Zonca. Planck intermediate results. XLVI. Reduction of large-scale systematic effects in HFI polarization maps and estimation of the reionization optical depth. A\&A, 596:A107, December 2016g. doi: 10.1051/0004-6361/201628890.

Planck Collaboration, Aghanim, N., Arnaud, M., Ashdown, M., Aumont, J., Baccigalupi, C., Banday, A. J., Barreiro, R. B., Bartlett, J. G., Bartolo, N., Battaner, E., Benabed, K., Benot, A., Benoit-Lvy, A., Bernard, J.-P., Bersanelli, M., Bielewicz, P., Bock, J. J., Bonaldi, A., Bonavera, L., Bond, J. R., Borrill, J., Bouchet, F. R., Boulanger, F., Bucher, M., Burigana, C., Butler, R. C., Calabrese, E., Cardoso, J.-F., Catalano, A., Challinor, A., Chiang, H. C., Christensen, P. R., Clements, D. L., Colombo, L. P. L., Combet, C., Coulais, A., Crill, B. P., Curto, A., Cuttaia, F., Danese, L., Davies, R. D., Davis, R. J., de Bernardis, P., de Rosa, A., 
de Zotti, G., Delabrouille, J., Dsert, F.-X., Di Valentino, E., Dickinson, C., Diego, J. M., Dolag, K., Dole, H., Donzelli, S., Dor, O., Douspis, M., Ducout, A., Dunkley, J., Dupac, X., Efstathiou, G., Elsner, F., Enlin, T. A., Eriksen, H. K., Fergusson, J., Finelli, F., Forni, O., Frailis, M., Fraisse, A. A., Franceschi, E., Frejsel, A., Galeotta, S., Galli, S., Ganga, K., Gauthier, C., Gerbino, M., Giard, M., Gjerlw, E., Gonzlez-Nuevo, J., Grski, K. M., Gratton, S., Gregorio, A., Gruppuso, A., Gudmundsson, J. E., Hamann, J., Hansen, F. K., Harrison, D. L., Helou, G., Henrot-Versill, S., Hernndez-Monteagudo, C., Herranz, D., Hildebrandt, S. R., Hivon, E., Holmes, W. A., Hornstrup, A., Huffenberger, K. M., Hurier, G., Jaffe, A. H., Jones, W. C., Juvela, M., Keihnen, E., Keskitalo, R., Kiiveri, K., Knoche, J., Knox, L., Kunz, M., Kurki-Suonio, H., Lagache, G., Lhteenmki, A., Lamarre, J.-M., Lasenby, A., Lattanzi, M., Lawrence, C. R., Le Jeune, M., Leonardi, R., Lesgourgues, J., Levrier, F., Lewis, A., Liguori, M., Lilje, P. B., Lilley, M., Linden-Vrnle, M., Lindholm, V., Lpez-Caniego, M., Macas-Prez, J. F., Maffei, B., Maggio, G., Maino, D., Mandolesi, N., Mangilli, A., Maris, M., Martin, P. G., Martnez-Gonzlez, E., Masi, S., Matarrese, S., Meinhold, P. R., Melchiorri, A., Migliaccio, M., Millea, M., Mitra, S., Miville-Deschłnes, M.-A., Moneti, A., Montier, L., Morgante, G., Mortlock, D., Mottet, S., Munshi, D., Murphy, J. A., Narimani, A., Naselsky, P., Nati, F., Natoli, P., Noviello, F., Novikov, D., Novikov, I., Oxborrow, C. A., Paci, F., Pagano, L., Pajot, F., Paoletti, D., Partridge, B., Pasian, F., Patanchon, G., Pearson, T. J., Perdereau, O., Perotto, L., Pettorino, V., Piacentini, F., Piat, M., Pierpaoli, E., Pietrobon, D., Plaszczynski, S., Pointecouteau, E., Polenta, G., Ponthieu, N., Pratt, G. W., Prunet, S., Puget, J.-L., Rachen, J. P., Reinecke, M., Remazeilles, M., Renault, C., Renzi, A., Ristorcelli, I., Rocha, G., Rossetti, M., Roudier, G., Rouill dOrfeuil, B., Rubio-Martn, J. A., Rusholme, B., Salvati, L., Sandri, M., Santos, D., Savelainen, M., Savini, G., Scott, D., Serra, P., Spencer, L. D., Spinelli, M., Stolyarov, V., Stompor, R., Sunyaev, R., Sutton, D., Suur-Uski, A.-S., Sygnet, J.-F., Tauber, J. A., Terenzi, L., Toffolatti, L., Tomasi, M., Tristram, M., Trombetti, T., Tucci, M., Tuovinen, J., Umana, G., Valenziano, L., Valiviita, J., Van Tent, F., Vielva, P., Villa, F., Wade, L. A., Wandelt, B. D., Wehus, I. K., Yvon, D., Zacchei, A., and Zonca, A. Planck 2015 results. XI. CMB power spectra, likelihoods, and robustness of parameters. A\&A, 594:A11, September 2016h. doi: 10.1051/0004-6361/201526926.

Poletti, D., Fabbian, G., Le Jeune, M., Peloton, J., Arnold, K., Baccigalupi, C., Barron, D., Beckman, S., Borrill, J., Chapman, S., Chinone, Y., Cukierman, A., Ducout, A., Elleflot, T., Errard, J., Feeney, S., Goeckner-Wald, N., Groh, J., Hall, G., Hasegawa, M., Hazumi, M., Hill, C., Howe, L., Inoue, Y., Jaffe, A. H., Jeong, O., Katayama, N., Keating, B., Keskitalo, R., Kisner, T., Kusaka, A., Lee, A. T., Leon, D., Linder, E., Lowry, L., Matsuda, F., Navaroli, M., Paar, H., Puglisi, G., Reichardt, C. L., Ross, C., Siritanasak, P., Stebor, N., Steinbach, B., Stompor, R., Suzuki, A., Tajima, O., Teply, G., and Whitehorn, N. Making maps of cosmic microwave background polarization for b-mode studies: the polarbear example. $A \mathcal{E} A$, 600:A60, 2017. doi: 10.1051/0004-6361/201629467. URL https : //doi .org/10.1051/0004-6361/201629467.

G. Prézeau and M. Reinecke. Algorithm for the Evaluation of Reduced Wigner Matrices. ApJS, 190: 267-274, October 2010. doi: 10.1088/0067-0049/190/2/267.

S. Prunet, P. A. R. Ade, J. J. Bock, J. R. Bond, J. Borrill, A. Boscaleri, K. Coble, B. P. Crill, P. D. Bernardis, G. D. Gasperis, G. D. Troia, P. C. Farese, P. G. Ferreira, K. Ganga, M. Giacometti, E. Hivon, V. V. Hristov, A. Iacoangeli, A. H. Jaffe, A. E. Lange, L. Martinis, S. Masi, P. Mason, P. D. Mauskopf, A. Melchiorri, L. Miglio, T. Montroy, C. B. Netterfield, E. Pascale, F. Piacentini, D. Pogosyan, F. Pongetti, S. Prunet, S. Rao, G. Romeo, J. E. Ruhl, F. Scaramuzzi, D. Sforna, and N. Vittorio. Noise Estimation in CMB Time-Streams and Fast Iterative Map-Making. In A. J. Banday, S. Zaroubi, and M. Bartelmann, editors, Mining the Sky, page 421, 2001. doi: 10.1007/10849171_53.

M. Remazeilles, A. J. Banday, C. Baccigalupi, S. Basak, A. Bonaldi, G. De Zotti, J. Delabrouille, C. Dickinson, H. K. Eriksen, J. Errard, R. Fernandez-Cobos, U. Fuskeland, C. Hervías-Caimapo, M. López-Caniego, E. Martinez-González, M. Roman, P. Vielva, I. Wehus, A. Achucarro, P. Ade, R. Allison, M. Ashdown, M. Ballardini, R. Banerji, N. Bartolo, J. Bartlett, D. Baumann, M. Bersanelli, M. Bonato, J. Borrill, F. Bouchet, F. Boulanger, T. Brinckmann, M. Bucher, C. Burigana, A. Buzzelli, Z.-Y. Cai, M. Calvo, C.-S. Carvalho, G. Castellano, A. Challinor, J. Chluba, S. Clesse, I. Colantoni, A. Coppolecchia, M. Crook, G. D’Alessandro, P. de Bernardis, G. de Gasperis, J.-M. Diego, E. Di 
Valentino, S. Feeney, S. Ferraro, F. Finelli, F. Forastieri, S. Galli, R. Genova-Santos, M. Gerbino,

J. González-Nuevo, S. Grandis, J. Greenslade, S. Hagstotz, S. Hanany, W. Handley,

C. Hernandez-Monteagudo, M. Hills, E. Hivon, K. Kiiveri, T. Kisner, T. Kitching, M. Kunz,

H. Kurki-Suonio, L. Lamagna, A. Lasenby, M. Lattanzi, J. Lesgourgues, A. Lewis, M. Liguori,

V. Lindholm, G. Luzzi, B. Maffei, C. J. A. P. Martins, S. Masi, D. McCarthy, J.-B. Melin, A. Melchiorri,

D. Molinari, A. Monfardini, P. Natoli, M. Negrello, A. Notari, A. Paiella, D. Paoletti, G. Patanchon,

M. Piat, G. Pisano, L. Polastri, G. Polenta, A. Pollo, V. Poulin, M. Quartin, J.-A. Rubino-Martin, L. Salvati,

A. Tartari, M. Tomasi, D. Tramonte, N. Trappe, T. Trombetti, C. Tucker, J. Valiviita, R. Van de Weijgaert,

B. van Tent, V. Vennin, N. Vittorio, K. Young, and for the CORE collaboration. Exploring Cosmic Origins with CORE: B-mode Component Separation. ArXiv e-prints, April 2017.

M. Shimon, B. Keating, N. Ponthieu, and E. Hivon. CMB polarization systematics due to beam asymmetry: Impact on inflationary science. Phys.Rev.D, 77(8):083003, April 2008. doi: 10.1103/PhysRevD.77.083003.

R. Stompor, A. Balbi, J. D. Borrill, P. G. Ferreira, S. Hanany, A. H. Jaffe, A. T. Lee, S. Oh, B. Rabii, P. L. Richards, G. F. Smoot, C. D. Winant, and J.-H. P. Wu. Making maps of the cosmic microwave background: The MAXIMA example. Phys.Rev.D, 65(2):022003, January 2002. doi: 10.1103/PhysRevD.65.022003.

M. Tomasi. dacapo_calibration: Photometric calibration code. Astrophysics Source Code Library, December 2016.

M. Tristram, C. Filliard, O. Perdereau, S. Plaszczynski, R. Stompor, and F. Touze. Iterative destriping and photometric calibration forplanck-HFi, polarized, multi-detector map-making. Astronomy $\mathcal{E}$ Astrophysics, 534:A88, Oct 2011. ISSN 1432-0746. doi: 10.1051/0004-6361/201116871. URL http://dx.doi.org/10.1051/0004-6361/201116871.

E. L. Wright, G. Hinshaw, and C. L. Bennett. Producing Megapixel Cosmic Microwave Background from Differential Radiometer Data. ApJ, 458:L53, February 1996. doi: 10.1086/309927. 